Modernisierung des öffentlichen Sektors | Standard 45

Nicolai Dose | Felix Wolfes | Carolin Burmester

\title{
Kleinstaaterei im Dienstrecht der deutschen Bundesländer
}

Probleme bei der Bundesländergrenzen überschreitenden Mobilität von Landesbeamten nach der Föderalismusreform I 


\section{Modernisierung des öffentlichen Sektors}

herausgegeben von / edited by

Jörg Bogumil, geschäftsführender Herausgeber,

Ruhr-Universität Bochum, Fakultät für Sozialwissenschaft

Julia Fleischer,

Universität Potsdam, Lehrstuhl für Politik und Regieren in Deutschland

Gisela Färber,

Hochschule für Verwaltungswissenschaften, Speyer

Wolfgang Gerstelberger,

Tallinn University of Technology, Department of Business Administration

Gerhard Hammerschmid,

Hertie School of Governance, Berlin, Public \& Financial Management

Marc Hansmann,

Mitglied des Vorstands der enercity AG Hannover

Sabine Kuhlmann,

Universität Potsdam, Lehrstuhl für Politikwissenschaft,

Verwaltung und Organisation

Renate E. Meyer,

Wirtschaftsuniversität Wien, Institut für Public Management

Erika Mezger,

Former Deputy Director, European Foundation for the Improvement of Living and Working Conditions (Eurofound), Dublin

Frieder Naschold $t$,

Wissenschaftszentrum Berlin für Sozialforschung

Christina Schaefer,

Helmut Schmidt-Universität Hamburg, Professur für Verwaltungs-

wissenschaft, insbesondere Steuerung öffentlicher Organisationen

Karsten Schneider,

Beigeordneter für Soziales, Bildung, Kultur und Sport bei der Stadt Herten

Göttrik Wewer,

Verwaltungs- und Politikwissenschaftler, Staatssekretär a.D.

Band 45 
Nicolai Dose | Felix Wolfes | Carolin Burmester

\section{Kleinstaaterei im Dienstrecht der deutschen Bundesländer}

Probleme bei der Bundesländergrenzen überschreitenden Mobilität von Landesbeamten nach der Föderalismusreform I 
Die Veröffentlichung basiert auf Ergebnissen des Forschungsprojekts „Die Föderalismusreform und die Beamten-Auswirkungen der jüngsten Reformen des deutschen Föderalismus" unter Leitung von Prof. Dr. Nicolai Dose, das mit Mitteln der HansBöckler-Stiftung gefördert wurde. Die Ergebnisse wurden für diese Veröffentlichung aktualisiert.

Die Deutsche Nationalbibliothek verzeichnet diese Publikation in der Deutschen Nationalbibliografie; detaillierte bibliografische Daten sind im Internet über http://dnb.d-nb.de abrufbar.

\author{
1. Auflage 2020 \\ () Nicolai Dose | Felix Wolfes | Carolin Burmester \\ Publiziert von \\ Nomos Verlagsgesellschaft $\mathrm{mbH} \& \mathrm{Co} . \mathrm{KG}$ \\ Waldseestraße 3-5 | 76530 Baden-Baden \\ www.nomos.de \\ Gesamtherstellung: \\ Nomos Verlagsgesellschaft mbH \& Co. KG \\ Waldseestraße 3-5 | 76530 Baden-Baden \\ ISBN (Print): 978-3-8487-6644-4 \\ ISBN (ePDF): 978-3-7489-0723-7 \\ DOI: https://doi.org/10.5771/9783748907237
}

\title{
(c) $(\Theta \Theta \Theta$
}

Dieses Werk ist lizenziert unter einer

Creative Commons Namensnennung - Nicht kommerziell Keine Bearbeitungen 4.0 International Lizenz.

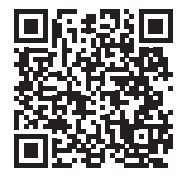

Onlineversion Nomos elibrary 


\section{Inhalt}

\section{Abbildungsverzeichnis}

Vorwort

1. Einleitung

1.1 Thema und Forschungsfragen

1.2 Ablauf einer Versetzung

1.3 Der Gang des Arguments

2. Forschungsdesign und angewendete Methoden

2.1 Die Online-Umfrage: Forschungsdesign, Fragebogen, Sample und Rücklauf

2.2 Die Fallstudien: Forschungsdesign und Fragebogen

3. Besoldungsunterschiede im Ländervergleich: potenzieller Anreiz für einen Wechsel des Bundeslandes

4. Vorschriftenanalyse

4.1 Die Neuregelung der Laufbahnsysteme

4.2 Mobilitätsanreize und -hemmnisse für Beamte der allgemeinen Verwaltung

4.3 Mobilitätsanreize und -hemmnisse für Polizisten

4.3.1 Laufbahngruppen der Polizei vor und nach der Föderalismusreform I

4.3.2 Nichtrechtlich-normierte Absprachen der IMK

4.4 Mobilitätsanreize und -hemmnisse für Lehrer

4.5 Thesen und Hypothesen

46 
5. Online-Umfrage 51

5.1 Mobilitätsmotive $\quad 52$

5.2 Mobilitätshemmnisse und -hürden 56

5.2.1 Mögliche Ablehnungsgründe auf Seiten der aufnehmenden Dienstbehörden $\quad 56$

5.2.2 Mögliche Hemmnisse auf Seiten der Beamten $\quad 60$

5.2.3 Tauschpartner 63

5.2.4 Laufbahnsysteme 66

5.3 Anerkennung von Ausbildung $\quad 68$

$\begin{array}{ll}5.4 \text { Wechselprobleme im Zeitverlauf } & 71\end{array}$

$\begin{array}{ll}\text { 6. Fallstudien } & 73\end{array}$

6.1 Motivation für einen Wechsel 74

6.2 Probleme bei einem Länder-Länder-Wechsel 76

$\begin{array}{ll}\text { 6.2.1 Probleme aus Landesgegebenheiten } & 77\end{array}$

6.2.2 Probleme durch das Wechselverfahren 80

6.2.2.1 Wechselregime bei der allgemeinen
Verwaltung

6.2.2.2 Wechselregime bei der Polizei 81

6.2.2.3 Wechselregime bei den verbeamteten
Lehrkräften

6.3 Intransparenz und Ungewissheit als belastende Faktoren $\quad 85$

6.4 Mobilitätsfördernde Faktoren bei einem Bundesländer übergreifenden Wechsel $\quad 86$

7. Handlungsempfehlungen $\quad 89$

8. Zusammenfassung und Fazit 93

$\begin{array}{ll}\text { Literatur } & 103\end{array}$

$\begin{array}{ll}\text { Autorinnen und Autoren } & 107\end{array}$ 


\section{Abbildungsverzeichnis}

Abbildung 1: $\quad$ Forschungslogik der Fallstudien

Abbildung 2: $\quad$ A 7-Jahresbruttobesoldung der Beamten der Länder und Kommunen bei Annahme einer 40-Stunden-Woche im Vergleich der Bundesländer im Jahr 2019

Abbildung 3: A 9-Jahresbruttobesoldung der Beamten der Länder und Kommunen bei Annahme einer 40-Stunden-Woche im Vergleich der Bundesländer im Jahr 2019

Abbildung 4: A 13-Jahresbruttobesoldung der Beamten der Länder und Kommunen bei Annahme einer 40-Stunden-Woche im Vergleich der Bundesländer im Jahr 2019

Abbildung 5: Gründe für einen Bundeslandwechsel (Mehrfachnennungen möglich)

Abbildung 6: $\quad$ Hauptgrund für einen Bundeslandwechsel (Ohne Mehrfachnennungen)

Abbildung 7: $\quad$ Berufliche und wirtschaftliche Gründe als Hauptmobilitätsanreiz

Abbildung 8: Institutionelle Gründe für die Ablehnung von Versetzungsanträgen seitens der Dienstbehörde (Mehrfachnennungen möglich)

Abbildung 9: Individuelle Gründe für die Ablehnung von Versetzungsanträgen seitens der Dienstbehörde (Mehrfachnennungen möglich)

Abbildung 10: Komplexität des Versetzungsverfahrens

Abbildung 11: Einschätzung: Finden eines Tauschpartners

Abbildung 12: Ausbildung wird voraussetzungslos anerkannt

Abbildung 13: $\quad$ Einstufung in eine niedrigere Besoldungsgruppe beim

Bundeslandwechsel 


\section{Vorwort}

Das dem vorliegenden Band zugrundliegende Projekt „Die Föderalismusreformen und die Beamten“ wurde unter der Projekt Nummer 2014-738-4 von der Hans-Böckler-Stiftung gefördert. Hierfür und für die Durchführung mehrerer Sitzungen des wissenschaftlichen Beirats sind wir der Stiftung und der uns betreuenden Dr. Dorothea Voss sehr dankbar. Groß ist auch unser Dank an die Mitglieder des wissenschaftlichen Beirats, die das Projekt stets konstruktiv begleitet haben. Als sehr positiv haben wir dabei die große Resonanz sowie die hervorragende Zusammensetzung aus Vertretern von Wissenschaft und Praxis empfunden. Sie haben uns mit vielen wichtigen Hinweisen und Fragen geholfen, den relevanten Aspekten auf sinnvolle Weise nachzugehen und stets eine hohe Qualität der Arbeit sicherzustellen. Wichtig war uns auch die Bestätigung, auf dem richtigen Weg zu sein.

Empirische Verwaltungsforschung steht wiederkehrend vor der Herausforderung, einen Zugang zum Forschungsfeld zu finden. Hier waren so manche Hürden zu überwinden, die von einigen vorgesetzten Stellen aufgestellt wurden. Umso mehr freuen wir uns, dass so viele Personalverantwortliche an unserer Online-Umfrage teilgenommen haben. Noch mehr Zeit, Energie und Reflexion haben die im Zuge der durchgeführten 32 Fallstudien befragten Personen aufgebracht. Hierfür danken wir insbesondere.

Allen am Thema interessierten wünschen wir eine ertragreiche Lektüre. Auf frühere Veröffentlichungen haben wir bereits Reaktionen von Betroffenen erfahren, denen der Wechsel von einem Bundesland in ein anderes erschwert oder gar verwehrt wird. Dieses Feedback hat uns gezeigt, wie nahe wir mit unserer Studie an der Lebenswirklichkeit von Beamtinnen und Beamten sind. Wir hoffen sehr, dass wir mit den vorgelegten Ergebnissen zu einer Verbesserung ihrer Situation beitragen können.

Duisburg und Münster im Februar 2020 Nicolai Dose, Felix Wolfes und Carolin Burmester 


\section{Einleitung}

Einleitend werden im Folgenden das Thema dieser Studie entwickelt und die Forschungsfragen formuliert. Da die Studie die Bundesländergrenzen überschreitende Mobilität von Beamten zum Gegenstand hat, also den Wechsel eines Beamten ${ }^{1}$ von einem Bundesland in ein anderes, soll hier auch der Ablauf einer solchen Versetzung vor die Klammer gezogen werden, um die nachfolgenden Ausführungen von diesem eher technisch-formalen Aspekt zu entlasten. Die Einleitung endet mit einer Skizze des Gangs des Arguments.

\subsection{Thema und Forschungsfragen}

Mit dem Inkrafttreten der Föderalismusreform I im Jahre 2006 schlug Deutschland endgültig den Weg in Richtung eines Trennföderalismus ein (siehe Häberle 2007, S. 46). Dieser ist von originären Länderzuständigkeiten geprägt, welche die Bundesländer je nach Politikvorstellung und je nach Finanzlage recht unterschiedlich ausfüllen können. Zu den Zuständigkeiten, die den Ländern zusätzlich übertragen wurden, gehört auch die Gesetzgebungskompetenz für Laufbahnen, Besoldung und Versorgung der Landesund Kommunalbeamten sowie der Beamten der anderen Körperschaften, Anstalten und Stiftungen des öffentlichen Rechts auf dem jeweiligen Landesgebiet (Jarass/Pieroth 2011, Art. 74 Abs. 1 Nr. 27 GG Rn. 77). Die Gesetzgebungskompetenz für die Bundesbeamten verbleibt nach Art. 73 Abs. 1 Nr. 8 GG beim Bund, die für die Statusrechte und -pflichten aller Beamten wechselte aus der abgeschafften Rahmengesetzgebungskompetenz in die Gegenstände der konkurrierenden Bundesgesetzgebung. Da die Länder die neu hinzugewonnenen Gesetzgebungszuständigkeiten im Rahmen insbesondere der Normierungen des Art. 33 GG selbstständig ausfüllen können (siehe Seeck/Rieger 2011, S. 1), entwickeln sich sowohl das Laufbahnrecht als auch die Besoldung in den verschiedenen Bundesländern in letzter Zeit zunehmend auseinander.

Es wäre jedoch falsch, den Beginn der Reföderalisierung im Beamtenbereich zu eng mit dem Inkrafttreten der Föderalismusreform I zu verknüpfen. Denn ein entsprechender Trend war bereits vorher auszumachen, wie

1 Um eine bessere Lesbarkeit des Textes zu gewährleisten, wird im Folgenden auf eine geschlechterneutrale Schreibweise verzichtet. 
Berndt Keller (2010, S. 132 f.) unter Verweis auf das im Jahre 2002 in Kraft getretene Gesetz zur Modernisierung der Besoldungsstruktur zutreffend argumentiert. $\mathrm{Zu}$ nennen wären in diesem Zusammenhang auch weitere Maßnahmen, mit denen seit Mitte der neunziger Jahre des zwanzigsten Jahrhunderts angestrebt wurde, die Regelungskompetenzen in diesem Bereich auf die Bundesländer zurück zu verlagern. Zu verweisen wäre hier gleichermaßen auf das Dienstrechtsreformgesetz (1997), das Gesetz zur Reform der Professorenbesoldung (2002) und das Bundesbesoldungs- und Bundesversorgungsanpassungsgesetz 2003/2004 (siehe Becker/Tepke 2011, S.326f.). Trotz dieser der Föderalismusreform I vorgelagerten Tendenzen zur Reföderalisierung erlangten diese ab dem Jahre 2006 eine neue Qualität. Karsten Schneider (2016, S. 17) spricht gar von einem „Paradigmenwechsel“. Dieser zeigte sich schon bald nach dem Inkrafttreten der Reform. Die Länder machten von den neuen Gesetzgebungskompetenzen relativ schnell Gebrauch und gestalteten sowohl die Besoldung als auch das Laufbahnrecht unter Berücksichtigung der jeweiligen länderspezifischen Gegebenheiten nach den je eigenen Vorstellungen (siehe Reintjes 2018). Die entsprechenden gesetzgeberischen Aktivitäten führten zu sich zunehmend auseinanderentwickelnden Besoldungshöhen in den Bundesländern (siehe DGB Bundesvorstand 2016; Schneider 2016, S. 17) sowie zu einem verstärkt fragmentierten Laufbahnrecht (siehe Dose 2013, S. 491 f.; Burmester 2015; Dose/ Reus 2016, S. 628, S. 637). Die Vorschriften zu den Laufbahnsystemen in den Ländern haben sich bereits dreizehn Jahre nach der entsprechenden Ermächtigung der Landesgesetzgeber deutlich auseinanderentwickelt.

Das zunehmend fragmentierte Dienstrecht trifft auf eine Entwicklung, die in den letzten Jahren verstärkt den gesamten Arbeitsmarkt erfasst hat. Es ist offensichtlich, dass die demographische Entwicklung mit der zunehmenden Verrentung/Pensionierung der Baby-Boomer und den zahlenmäßig sehr viel schwächeren nachrückenden Jahrgängen nahezu jede Branche vor massive Nachwuchssorgen stellt. Gerade der öffentliche Dienst ist hiervon in besonderer Weise betroffen, weil in nicht wenigen Verwaltungen die Jahrgänge nach den Babyboomern wegen des ca. 1990 einsetzenden und bis ins Jahr 2008 reichenden Personalabbaus in der öffentlichen Verwaltung relativ schwach besetzt sind. Die Zahl der erfahrenen Mitarbeiter wird folglich dramatisch abnehmen. Denn 27 Prozent der im öffentlichen Dienst Beschäftigten sind mindestens 55 Jahre alt; im Durchschnitt aller Erwerbstätigen liegt die Quote bei 24 Prozent. Es wird mit einer Zahl von 1,5 Mio. Beschäftigten des öffentlichen Dienstes gerechnet, die bis zum Jahre 2030 in den Ruhestand gehen (Riedel/Specht 2020, S. 6). Dabei sind schon jetzt freiwerdende und neu geschaffene Stellen nicht mehr zu besetzen. Je nach Schätzung fehlen im Öffentlichen Dienst zwischen 200.000 und 300.000 Mitarbeiter*in- 
nen ${ }^{2}$, wobei es die Kommunen mit einem Personalbedarf von nahezu 140.000 Stellen am härtesten trifft. Aber auch in der Pflege, im Schuldienst, bei der Bundes- und Landespolizei ${ }^{3}$ sowie in der Finanzverwaltung klaffen dramatische Personallücken. Selbst in den Bundesministerien bleiben Schreibtische verweist. Wie eine Anfrage im Deutschen Bundestag ergab, sind bspw. im Innenministerium 266 Stellen, im Außenministerium 265 Stellen, im Verteidigungsministerium 154 Stellen und im Finanzministerium 150 Stellen unbesetzt. Dass es sich nicht nur um vorübergehende Personallücken handelt, lässt sich an dem Umstand ablesen, dass einige Stellen bereits über 12 Monate unbesetzt seien ${ }^{4}$.

Es ist also davon auszugehen, dass die Länder (und der Bund) erheblich unter Druck stehen, neue Mitarbeiter*innen zu attrahieren und bestehende zu halten. Das Schäuble-Wort vom „Wettbewerb um die besten Köpfe“ (DGB Bundesvorstand 2006a, S. 1) erhält damit eine ganz neue Dimension. Es stellen sich Fragen, wie die, ob der Wettbewerb zwischen den Bundesländern fair geführt werden kann, wenn sie sehr ungleiche finanzielle Voraussetzungen haben. Wohlhabende Länder wie bspw. Bayern sind hier auch angesichts der Schuldenbremse im Vorteil. Immerhin ist der Staatsschuldenquote eines Landes die wichtigste unabhängige Variable, wenn es darum geht, die unterschiedliche Höhe der Besoldung in den Ländern zu erklären (Dose und Wolfes 2016, S. 286 ff.). Und wenn es richtig ist, dass die Beamten dorthin gehen, wo sie am besten bezahlt werden, sind die Aussichten für die weniger gut besoldenden Länder nicht gut.

Gleichfalls ist die Frage nach den Wirkungen des ausdifferenzierten Laufbahnrechts auf die Bundesländergrenzen übergreifende Mobilität von Beamten von Bedeutung. Hierzu lässt sich einerseits festhalten, dass sich die Reformländer mit dem Potenzial zu einer erhöhten Binnenmobilität und verbesserten Aufstiegschancen ihrer Beamten präsentieren. Insbesondere besteht die breit genutzte Möglichkeit, bislang vor der Reform bestehende Hürden beim Aufstieg von einer Laufbahngruppe in die nächste abzubauen. So mussten insbesondere die Verzahnungsämter effektiv zweimal durch-

2 Öffentlicher Dienst: Es fehlen 300.000 Beschäftigte, tagesschau.de vom 06.01.2020, https://w ww.tagesschau.de/inland/oeffentlicher-dienst-mitarbeiter-101.html, zuletzt abgerufen am 11.01.2020. Riedel und Specht (2020, S.6) gehen von „mehr als 200000 Beschäftigte[n]“ aus.

3 Im Oktober 2019 meldete das Handelsblatt, dass die Polizei mehr als 7.000 Dienstschichten nicht durchgehend besetzen könne (Neuerer 2019).

4 Personallücken: Hunderte Stellen in Bundesministerien unbesetzt, Handelsblatt vom 15. Dezember 2019, https://www.handelsblatt.com/politik/deutschland/personalluecken-hu nderte-stellen-in-bundesministerien-unbesetzt/25337526.html, zuletzt abgerufen am 11.01.2020. 
laufen werden. Durch die Reduzierung der Laufbahngruppen lassen sich solche Restriktionen abmildern oder gar aufheben (vgl. Seeck/Rieger 2011, S. 8). Andererseits muss davon ausgegangen werden, dass die zunehmend unterschiedlich gestalteten Systeme die Mobilität von Beamten über Landesgrenzen hinweg erschweren.

Zwar liegt eine Reihe von Publikationen vor, in denen genau diesem zweiten Gedanken nachgegangen wird, nämlich, dass das sich auseinanderentwickelnde Laufbahnrecht der Länder zu einer verstärkten Mobilitätsbeschränkung der Beamten führt (Lorse 2010, S. 121; Schnellenbach 2011, $\mathbb{S} 1$ Rn. 8; Hoffmann 2012, S. 27 f.; Dose 2013, S. 492 f.). Allerdings liegt hierzu noch keine empirische, systematisch vorgehende Studie vor. Streng genommen bleibt es also bei einer Vermutung. Das Schließen dieser Forschungslücke ist durchaus relevant. Zwar dürften die Beamten wegen des prinzipiell lebenslang geltenden Beamtenrechtsverhältnisses keinen rechtlichen Anspruch auf einen Dienstherrenwechsel besitzen (Wolff 2011, S. 68), dennoch ist es gerade angesichts der weiter oben bereits angesprochenen stark unterschiedlich entwickelnden Besoldungshöhen (siehe auch Kapitel 3) aus Sicht der Beamten von allergrößter Bedeutung, dass ihnen die Möglichkeit bleibt, in diejenigen Bundesländer zu wechseln, welche die besten Beschäftigungsbedingungen bieten (vgl. Dose 2016, S. 36). Dabei muss hier als Anreiz zu verstärkter Mobilität nicht einseitig auf die Höhe der Besoldung abgehoben werden, sondern es können auch die Attraktivität des jeweiligen Laufbahnsystems oder der Umstand, dass Verbeamtungen erfolgen oder eben auch nicht (siehe Bochmann 2019, S. 154 f.), in die Betrachtung einbezogen werden. Auch die vom Dienstherrn stets betonte Möglichkeit der Familienzusammenführung macht möglichst geringe faktische Hürden beim Wechsel von einem Bundesland in ein anderes wünschenswert (KMK, 2001, S.2). Um tatsächlich von einem Bundesland in ein anderes wechseln zu können, dürfen keine oder nur geringe Mobilitätshemmnisse bestehen. Folglich sind die bereits bestehenden Hemmnisse, die beispielsweise aus unterschiedlichen Anforderungen an die formale Qualifikation entstehen, systematisch zu ermitteln. Würde sich darüber hinaus zeigen, dass die Differenzierung der Laufbahnsysteme ein wesentliches zusätzliches Mobilitätshemmnis darstellt, müsste die deutsche Praxis des Wettbewerbsföderalismus in Frage gestellt werden (vgl. Hannack 2013, S. 7). Denn zu diesem gehört auch, dass die vom Wettbewerb Betroffenen die Wahl haben, in welcher Gebietskörperschaft mit ihren je spezifischen Bedingungen sie leben wollen (Kropp 2010, S. 46; Dose 2016, S.39). Wäre den Beamten die regionale Mobilität aufgrund unterschiedlicher Laufbahnsysteme abgeschnitten, würde der Wettbewerb zwischen den Bundesländern tatsächlich auf dem Rücken der Beamten ausgetragen werden (vgl. Dose 2013). Aus den vorstehenden Überlegun- 
gen ergibt sich die Bedeutung der nachstehenden Forschungsfragen, die im Zentrum der hiermit vorgelegten Studie stehen:

Welches sind die stärksten Motive für einen Wechsel des Bundeslandes?

Bedeuten die sich inhaltlich auseinanderentwickelnden Laufbahngesetze in der Vollzugspraxis ein Mobilitätshemmnis für wechselwillige Beamte?

Dabei werden wir systematische Vergleiche anstellen können zwischen den neu föderalisierten Bereichen der allgemeinen Verwaltung und der Finanzverwaltung einerseits und den schon länger föderalisierten Bereichen von Polizei und schulischer Bildung andererseits. Wie im Methodenkapitel dargelegt wird, sind die Ergebnisse dieser Vergleiche vorsichtig zu interpretieren, weil der überwiegende Anteil der beantworteten Fragebögen der durchgeführten Onlineumfrage aus dem Bereich der allgemeinen Verwaltung stammt.

Nicht vorgenommen werden soll ein Vergleich zwischen den Bundesländern, sieht man von einer in Kapitel 3 vorgenommenen Analyse der unterschiedlichen Besoldungshöhen ab. Letztere dienen einem Verständnis von möglichen monetären Mobilitätsanreizen. Ein Vergleich zwischen Bundesländern verbietet sich, weil hierfür bei einer Rücklaufquote von 24,5 Prozent bei 1.376 befragten Stellen pro Bundesland eine zu geringe Zahl an rückgelaufenen Fragebögen verbleibt, um diese sinnvoll auswerten zu können. Es war auch zu keinem Zeitpunkt ein Bundesländervergleich der Ergebnisse der Online-Umfrage beabsichtigt.

Konzeptionell bedient sich die hier präsentierte Arbeit an der Forschungsheuristik des Akteurzentrierten Institutionalismus nach Mayntz und Scharpf in seiner Basisvariante (Mayntz/Scharpf 1995). Grundlegend sind damit Institutionen in einem neo-institutionalistischen Verständnis als restringierender, aber auch als ermöglichender „Handlungskontext“ (Mayntz/ Scharpf 1995, S. 43, Hervorh. im Original). Innerhalb dieses Handlungskontextes interagieren die verschiedenen Akteure vor dem Hintergrund ihrer jeweiligen Handlungsorientierungen (Mayntz/Scharpf 1995, S. 49 ff.). Das institutionelle Setting wird dabei durch die verfassungsrechtlichen Vorgaben des Grundgesetzes und vor allem durch die unterschiedlichen Dienstrechte einschließlich der Besoldung gesetzt. Wie bereits dargelegt können vom Dienstrecht grundsätzlich Anreize zu verstärkter Mobilität ausgehen, wenn beispielsweise die Zahl der Laufbahngruppen reduziert wird, so dass beim Aufstieg Verzahnungsämter nicht zweifach durchlaufen werden müssen und die aufwendigen Verfahren hierfür entfallen. Zusammen mit einer möglicherweise besseren Besoldung können hiervon Anreize für einen Bundeslandwechsel ausgehen. Wie wir zeigen werden, kann umgekehrt insbesondere das fragmentierte Dienstrecht wegen nicht kompatibler Vorschriften Bun- 
desländergrenzen überschreitende Mobilität zumindest behindern, womit dann der restringierende Kontext angesprochen ist. Mit Blick auf die Handlungsorientierung gehen wir von einer moderaten Nutzenmaximierung der Akteure aus, wobei jedoch auch mehr als rein monetäre Anreize einbezogen werden (vgl. Kathke 2018, passim). Tatsächlich kann hier mit Vorgriff auf die Ergebnisse darauf verwiesen werden, dass Mobilitätsbestrebungen stärker durch bspw. den Wunsch nach Nähe zur ursprünglichen Heimat als durch rein monetäre Besserstellungen ausgelöst werden (siehe Kapitel 5.1).

\subsection{Ablauf einer Versetzung}

Zur Entlastung der nachfolgenden Teile der Arbeit soll an dieser Stelle der typische Ablauf einer Versetzung skizziert werden. Damit soll Hintergrundwissen in der Hoffnung angeboten werden, ein besseres Verständnis der in den nachfolgenden Kapiteln präsentierten und diskutierten Ergebnisse zu ermöglichen.

Eine Versetzung ist im Innenverhältnis ein relativ komplizierter Prozess, an dem viele Akteure beteiligt sind. Dieser soll hier anhand einer fiktiven Versetzung von Hamburg in ein anderes Bundesland dargestellt werden. Das Vorgehen steht in diesem Fall auch stellvertretend für andere Bundesländer, obschon die Abläufe besonders im Innenverhältnis im Detail abweichen können. Das Verfahren ist in der Regel durch Verwaltungsvorschriften konkretisiert. Für eine Versetzung sind auf der obersten Normebene die Vorschriften des $\$ 15$ BeamtStG ${ }^{5}$ maßgeblich:

\section{$\$ 15$ Versetzung}

(1) Beamtinnen und Beamte können auf Antrag oder aus dienstlichen Gründen in den Bereich eines Dienstherrn eines anderen Landes oder des Bundes in ein Amt einer Laufbahn versetzt werden, für die sie die Befähigung besitzen.

(2) Eine Versetzung bedarf der Zustimmung der Beamtin oder des Beamten. Abweichend von Satz 1 ist die Versetzung auch obne Zustimmung zulässig, wenn das neue Amt mit mindestens demselben Grundgehalt verbunden ist wie das bisherige Amt. Stellenzulagen gelten hierbei nicht als Bestandteile des Grundgehalts.

(3) Die Versetzung wird von dem abgebenden im Einverständnis mit dem aufnehmenden Dienstherrn verfügt. Das Beamtenverhältnis wird mit dem neuen Dienstherrn fortgesetzt.

Über diese rechtlichen Vorgaben hinaus gibt es weitere Vereinbarungen zwischen den Bundesländern. So haben die Länder durch mehrere Beschlüsse

5 Beamtenstatusgesetz vom 17. Juni 2008 (BGBl. I S. 1010), das durch Artikel 15 Absatz 16 des Gesetzes vom 5. Februar 2009 (BGBl. I S. 160) geändert worden ist. 
der Innenministerkonferenz (IMK) festgelegt, dass ein Länder-Länder-Wechsel bei Polizeibeamten immer im Zuge eines Tauschverfahrens vollzogen werden soll. Entsprechendes hat die Kultusministerkonferenz (KMK) für den Lehramtsbereich vereinbart. Hier erfolgt in Anwendung der Beschlüsse der Wechsel entweder durch ein offenes Bewerbungsverfahren oder die Teilnahme an einem Lehrertauschverfahren.

Diese Beschlüsse sind jedoch gesetzlichen Regelungen nachrangig, sodass diese nur bei der Verhandlung zwischen aufnehmenden und abgebenden Dienstherrn Wirkung entfalten können. $\$ 15$ BeamtStG nennt zwei Tatbestände. Erstens kann der Beamte auf eigenen Antrag oder zweitens aus dienstlichen Gründen zu einem Dienstherrn in einem anderen Bundesland versetzt werden (siehe Reich 2012, S. 141 f.). In unserem Fall wird lediglich der Antragstatbestand behandelt, weil durch den Dienstherrn veranlasste Versetzungen nicht im Fokus der hier vorgelegten Studie stehen. Bei der anzuwendenden Vorschrift handelt es sich um eine Ermessensnorm, sodass dem Antragsteller nur ein Recht auf eine fehlerfreie Ermessensentscheidung zusteht, nicht jedoch ein Recht auf die Versetzung selbst. Nur bei einer Ermessensreduzierung auf null hätte der Ersuchende einen Anspruch auf die beantragte Versetzung. Wird eine Versetzung ohne die Zustimmung des abgebenden Dienstherrn angestrebt, ist dies ausschließlich durch eine erneute Ernennung beim neuen Dienstherrn möglich, wodurch nach $\$ 22$ Abs. 2 BeamtStG die Entlassung beim alten Dienstherrn kraft Gesetzes erfolgt. In diesem Fall muss der neue Dienstherr jedoch auch alle Pensionslasten tragen und verzichtet auf alle staatsvertraglich geregelten Ansprüche gegenüber dem alten Dienstherrn (siehe Rieger 2014, S. 178).

Für eine Versetzung nach $\ 15$ BeamtStG ist die Dienstbehörde im Außenverhältnis die oberste Dienstbehörde, also der Senat/das Personalamt (Rundschreiben des Personalamtes der Freien und Hansestadt Hamburg vom 18.09.2013). Diese übt auch das Ermessen aus. Sie führt jedoch in Hamburg nicht die Verhandlungen mit dem aufnehmenden Dienstherrn. Im Innenverhältnis übernimmt dies die Beschäftigungsdienststelle. Der Antrag auf Versetzung wird auf dem Dienstweg bei der Beschäftigungsdienststelle gestellt. Diese nimmt zur Vorabklärung Kontakt mit dem aufzunehmenden Dienstherrn auf, bei dem die internen Verfahren damit ebenso nach dessen Verwaltungsvorschriften angestoßen werden. Wenn sich aufnehmender und abgebender Dienstherr über die Details der Versetzung, wie beispielsweise über den Tauschpartner einig sind, gibt die Beschäftigungsbehörde den Vorgang zur Entscheidung mit dem Votum für die Versetzung an die oberste Dienstbehörde ab. Diese ist nicht an den Entscheidungsvorschlag der Beschäftigungsbehörde gebunden, folgt diesem aber in der Regel. Die oberste Dienstbehörde holt nun offiziell die Zustimmung beim aufneh- 
menden Dienstherrn nach $₫ 15$ Abs. 3 BeamtStG ein (siehe hierzu Rieger 2014, S. 173 f., 182 und auch Reich 2012, S. 146 f.). Nachdem die Zustimmung erklärt wurde, erfolgt eine Entscheidung unter Berücksichtigung der Stellungnahme des Personalrates (siehe Rieger 2014, S. 182, 174). Die Entscheidung geht dem Antragsteller in Form eines Verwaltungsaktes zu. Bei einer positiven Entscheidung wird das Beamtenverhältnis beim neuen Dienstherrn fortgesetzt (siehe hierzu Rieger 2014, S. 181 und auch Reich 2012, S. 147).

Gegen einen ablehnenden oder ungünstigen Verwaltungsakt kann der Antragssteller Rechtsmittel einlegen. Grundsätzlich gilt das jedoch nur für den endgültigen Bescheid. Gegen Zwischenschritte, wie z. B. die Verweigerung der Zustimmung des aufnehmenden Dienstherrn bei der Vorabklärung kann - da es sich hier um eine verwaltungsinterne Angelegenheit handelt - kein Rechtsmittel eingelegt werden. Eine Ausnahme bildet die Entscheidung der Beschäftigungsstelle, die Versetzung nicht weiter zu verfolgen. Es liegt damit zwar kein widerspruchsfähiger Verwaltungsakt vor, dennoch ist diese Entscheidung nach $₫ 54$ BeamtStG widerspruchsfähig. Außerdem ist in den meisten Beamtengesetzen der Bundesländer der nichtförmliche Beschwerdeweg zur obersten Dienstbehörde gegeben. In Hamburg ist dies in $\$ 101 \mathrm{HmbBG}$ geregelt (siehe Rieger 2016 und auch Rieger 2014, S. 182).

\subsection{Der Gang des Arguments}

Die Beantwortung der aufgeworfenen Forschungsfragen soll in vier Schritten erfolgen. In einem ersten Schritt werden das zugrunde gelegte Forschungsdesign sowie die eingesetzten Methoden erläutert (Kapitel 2). Es wird deutlich werden, dass methodisch auf eine Triangulation (between method) zurückgegriffen wurde (siehe Behnke/Baur/Behnke 2006, S. 125). Am Beginn des empirischen Teils steht eine Analyse der sich unterschiedlich entwickelnden Besoldungshöhen im Vergleich der Bundesländer für die Besoldungsgruppen A 7, A 9 und A13 (Kapitel 3). Es wird deutlich, dass die Besoldungshöhen zwischen den Bundesländern ganz erheblich variieren, es also ein gewisser, allerdings rein monetärer Anreiz für einen Bundeslandwechsel besteht. Hieran schließt sich die Untersuchung der Vorschriften des relevanten Beamtenrechts in den verschiedenen Bundesländern an, um weitere, nun in der Rechtsordnung angelegte Anreize für einen Wechsel, aber auch mögliche Hindernisse aufzudecken (Kapitel 4). Anschließend werden die auf diese Weise und durch eine systematische Literaturauswertung gewonnenen Erkenntnisse verdichtet (siehe Kapitel 4.5) und im Rahmen zweier ver- 
schiedener, sich gegenseitig ergänzender Ansätze zur Datengewinnung auf ihre empirische Gültigkeit geprüft: Erstens soll auf die Fachkompetenz und die Einschätzung aller für die Personalarbeit zuständigen Stellen der in den Fokus genommenen Teile der staatlichen Verwaltung aller Bundesländer im Rahmen einer Online-Befragung zurückgegriffen werden. Die Ergebnisse dieser Befragung werden in Kapitel 5 präsentiert. Zweitens wird auf einen Fallstudienansatz rekurriert, um weitere, tieferliegende Zusammenhänge aufdecken zu können. Er dient sowohl der Überprüfung und Validierung des bis dorthin gewonnenen Wissens als auch der Generierung neuer, ergänzender Thesen. Die wichtigsten, durch die Auswertung von insgesamt 32 Fallstudien gewonnenen Ergebnisse werden in Kapitel 6 dargelegt und diskutiert. Abschließend sollen in Kapitel 7 Handlungsempfehlungen formuliert sowie in Kapitel 8 eine Zusammenfassung erfolgen und ein Fazit gezogen werden. 


\section{Forschungsdesign und angewendete Methoden}

Die hier dokumentierte Studie folgt weitestgehend einem Querschnittsdesign. Methodisch wird zur Beantwortung der Forschungsfragen auf eine Triangulation (siehe Behnke/Baur/Behnke 2006, S. 125) gesetzt. Dabei gingen wir davon aus, dass die Informations- und Erkenntnisdichte durch dieses Vorgehen gesteigert werden kann. Zur Durchführung der Vorschriftenanalyse wird dokumenten-analytisch angesetzt, die Online-Umfrage beruht auf dem gängigen Instrumentarium der Umfrageforschung und die Fallstudien rekurrieren sowohl auf quantitative als auch qualitative Forschungsvorstellungen.

\subsection{Die Online-Umfrage: Forschungsdesign, Fragebogen, Sample und Rücklauf}

Die Online-Befragung dient der quantitativ-empirischen Überprüfung und Ergänzung der Erkenntnisse aus der zuvor durchgeführten Vorschriftenanalyse. Anschließend werden die Ergebnisse mit denen aus der FallstudienAnalyse abgeglichen. Ausgangspunkt der empirischen Arbeiten war damit die systematische und breit ansetzende Analyse der in den verschiedenen Bundesländern geltenden Vorschriften des Laufbahnrechts mit dem Ziel, das $\mathrm{Ma} ß$ an Fragmentierung der Vorschriften im Ländervergleich greifbar zu machen und mögliche Inkompatibilitäten aufzudecken, die das Potenzial besitzen, als Hemmnis bei einem Bundesländergrenzen überschreitenden Wechsel eines Beamten wirken zu können.

Da die bloße Analyse der Vorschriften jedoch nicht ausreicht, um die tatsächlichen Auswirkungen der Föderalisierung auf die Vollzugspraxis beim Bundeslandwechsel zu erfassen, blieb die übergeordnete Forschungsfrage zunächst unbeantwortet. Die Vorschriftenanalyse lieferte jedoch die Regelungsbereiche, in denen es lohnend und sinnvoll erschien, die Wirkungen der Fragmentierung bei Experten abzufragen. Tatsächlich ist die durchgeführte Umfrage eine Expertenbefragung, bei welcher die Grundgesamtheit aus allen obersten Landesbehörden und den zugehörigen nachgeordneten Behörden besteht. Voraussetzung für die Teilnahme war eine Zuständigkeit für Polizeibeamte, Beamte der allgemeinen Verwaltung, Lehrer oder Finanzbeamte. Innere Sicherheit und Bildung waren bereits vor der Föderalismusreform Ländersache, sodass davon ausgegangen werden konnte, dass sich im Lehramts- und Polizeibereich bereits Folgen einer Föderalisierung 
eingestellt hatten. Diese Bereiche könnten also Hinweise geben, wie sich die Situation in den vor 2006 nicht oder nur wenig föderalisierten Bereichen aktuell oder in Zukunft darstellen könnte. Folglich liegt die Gruppe der Beamten der allgemeinen Verwaltung im eigentlichen Fokus der Online-Umfrage, da diese von der neuen Föderalisierung vermutlich am stärksten betroffen sind. Explorative Experteninterviews im Vorfeld der Umfrage haben ergeben, dass sich bei den Finanzbeamten bisher die geringsten Auswirkungen zeigen. Dennoch erscheint es sinnvoll, neben den Beamten der allgemeinen Verwaltung mit den Beamten der Finanzverwaltung Mitarbeiter eines weiteren, neu föderalisierten Verwaltungszweigs in die Analyse einzubeziehen, um die gewonnenen Ergebnisse breit abzusichern oder ggf. differenziert darstellen zu können.

Der verwendete Fragebogen besteht zum größten Teil aus standardisierten Fragen und zu einem sehr kleinen Teil aus offenen Fragen, die darauf zielen, mögliche in den standardisierten Fragen nicht abgebildete Erklärungsfaktoren und Einschätzungen aufzudecken. Am Anfang des Fragebogens steht eine Frage nach der Beamtengruppe, für den die befragte Person zuständig ist, um später eine entsprechend differenzierte Auswertung vornehmen zu können. Anschließend werden mögliche Gründe für den Wechsel des Bundeslandes abgefragt. Es folgen Fragenblöcke zu Versetzungsanträgen und Tauschpartnern, zu den möglichen Gründen des Scheiterns bzw. des Erfolgs von Versetzungsanträgen, zum Verfahren und dem möglichen Erfordernis sich nachschulen zu lassen sowie zu den Auswirkungen des fragmentierten Laufbahnsystems und der fragmentierten Vorschriften. Weitere Blöcke waren spezifisch an Personalverantwortliche gerichtet, die im Lehramtsbereich oder bei der Polizei tätig sind. Abschließend wurde noch nach Bundesland, Behördenebene und Funktion gefragt. Die Fragen konnten stets auf einer Fünfer-Skala des Likert-Typs mit einer „teils/teils“-Kategorie in der Mittellage beantwortet werden. Meist wurde direkt gefragt und gelegentlich sollten vorgegebene Sätze mit einem entsprechenden Attribut aus der vorgegebenen Skala zu Ende gebracht werden.

In Vorbereitung der Online-Umfrage sind vorab einige relevante Kriterien aus der Literatur zu diskutieren, von denen angenommen wird, dass sie zur Erhöhung der Datenqualität und des Rücklaufs beitragen; sie sind auch bei der hier präsentierten Befragung systematisch berücksichtigt worden. Zur Steigerung der Ausschöpfungsquote sei insbesondere eine vor der eigentlichen Befragung liegende Kontaktabnahme hilfreich. Ähnlich wirke eine persönliche Anrede der Befragten und ein- oder mehrmaliges Nachfassen, wenn nach der ersten Bitte um Beteiligung noch keine Resonanz zu verzeichnen ist (vgl. Maurer/Jandura 2009, S. 67). Auch sollte der Zeitaufwand für das Ausfüllen des Fragebogens gering gehalten werden und den zuvor 
gemachten Angaben entsprechen ${ }^{6}$, um Abbrüche und Frustrationen zu vermeiden. Antwortverweigerungen und Abbrüchen kann durch möglichst kurze, einfache Fragen und der Wiederholung von Skalenbeschriftungen entgegengewirkt werden (vgl. Pötschke 2009, S. 85 f). Ebenso wichtig, insbesondere bei der Befragung von Fachpublikum, ist eine seriöse grafische Darstellung des Umfragefensters mit schlichten Farben und die Möglichkeit, die Umfrage auch auf einem Tablet-PC oder einem Smartphone beantworten zu können (vgl. Maurer/Jandura 2009, S. 67 ff.).

Diesen grundsätzlichen Überlegungen folgend, wurden, noch bevor die eigentliche Online-Umfrage geschaltet und den befragten Stellen ein Link für den Zugang übermittelt wurde, jeweils die personalverantwortlichen Stellen mit der Bitte angeschrieben, dass pro Behörde eine Person aus der Personaleinheit an der Befragung teilnimmt. Aus dem Sample ausgeschlossen wurden die Behörden, welche sich selbst nach Rücksprache als nicht zuständig für Bundesländer übergreifende Dienstherrenwechsel bezeichneten oder als in der Thematik zu unerfahren abgesagt hatten. Diese können nicht als Experten gelten, weshalb deren Befragung als nicht sinnvoll erschien. Da alle zuständigen Stellen angeschrieben wurden, handelt es sich um eine Vollerhebung. Die benötigten Post- und E-Mail-Adressen wurden über Organigramme der Behörden bezogen, und fehlende Adressen über Staatshandbücher der Bundesländer ergänzt. Durch die Nutzung des Befragungstools EFS Survey konnten die Adressen so eingepflegt werden, dass zwei Nachfassaktionen durchgeführt werden konnten. Erneut angeschrieben wurden nur Personen, die noch nicht teilgenommen hatten. Gleichzeitig konnten bei diesem Verfahren Anonymität und Datenschutz gewährleistet werden.

Das Gesamtsample umfasst 1.376 Stellen; nach Beendigung der Feldphase betrug die Ausschöpfungsquote 24,5 Prozent. Die verwaltungsbereichsspezifischen Rücklaufquoten lassen sich nicht exakt bestimmen, weil die Zuordnung durch die Befragten erfolgte und nicht mit unserer Vorabfestlegung übereinstimmen muss. Nur auf Basis dieser Vorabfestlegung lässt sich jedoch die Grundgesamtheit festmachen, die jedoch von der Selbsteinschätzung der Beamten abweichen kann. Jede Berechnung der bereichsspezifischen Rücklaufquote wäre damit potenziell fehlerhaft, weshalb wir darauf verzichtet haben, sie zu berechnen. Es gibt jedoch keinen Anhaltspunkt für massiv ungleichgewichtige Rücklaufquoten. Da die allgemeine Verwaltung jedoch zahlenmäßig überwiegt, sind die absoluten Rücklaufzahlen bei den anderen Verwaltungsbereichen niedriger als für die allgemeine Verwal-

6 In den Anschreiben wurde die Beantwortungszeit auf etwa 12 Minuten geschätzt. Mit einer durchschnittlichen tatsächlichen Beantwortungszeit von 11 Minuten und 26 Sekunden lag diese im angegebenen Zeitrahmen. 
tung. Dies gibt Anlass für eine vorsichtige Interpretation der entsprechenden Ergebnisse. Bei der Zuordnung zu einer Behördenebene haben gut ein Drittel der Befragten keine Angabe gemacht. Von den Personen, die sich einer Ebene zugeordnet haben, waren 15,4 Prozent in einer obersten Landesbehörde tätig, 8,6 Prozent in einer Landesoberbehörde, 11,6 Prozent in einer Landesmittelbehörde, 20,8 Prozent in einer unteren Landesbehörde und 11,3 Prozent haben angegeben, dass sie auf einer anderen Ebene oder in einer Behörde mit einer anderen Bezeichnung tätig sind. Die Datenerhebung fand im Winter 2015/2016 statt. Die Feldzeit betrug etwa drei Monate.

\subsection{Die Fallstudien: Forschungsdesign und Fragebogen}

Ziel der durchgeführten Fallstudien war es, die unter der Oberfläche liegenden, nicht sogleich sichtbaren Wirkungszusammenhänge aufzudecken. Erst mit ihnen können die beamtenspezifischen Wirkungen der Föderalismusreform I wirklich offengelegt werden.

Abbildung 1 gibt die damit angesprochene Forschungslogik wieder: Auf einer oberen, sichtbaren Ebene steht als Ausgangspunkt die Föderalismusreform I des Jahres 2006. Wie dargestellt hat die sich in der Folge der Föderalismusreform einstellende Fragmentierung des Laufbahnrechts Konsequenzen für Bundesländergrenzen übergreifende Wechsel von Landesbeamten: Sie werden komplizierter und aufwendiger (siehe Lorse 2010, S. 121). Die Gründe hierfür sind jedoch nicht offensichtlich, sondern müssen für eine Betrachtung der tatsächlichen Hintergründe im Detail rekonstruiert werden. Bezieht man die in Abbildung 1 veranschaulichte Forschungslogik auf den einzelnen zu analysierenden Fall, zeigen sich Schwierigkeiten wie nicht rechtlich begründete Inkompatibilitäten, divergierendes Dienstrecht, fehlerhafte Anwendung von Recht sowie Probleme mit den Wechselverfahren. Die Schwierigkeiten können im Einzelfall so massiv sein, dass der angestrebte Wechsel scheitert oder erst gar nicht mehr versucht wird. Die Fallstudien bieten die Chance, die verschiedenen Erklärungsfaktoren aufzudecken und zueinander in Beziehung zu setzen. 


\section{Abbildung 1: Forschungslogik der Fallstudien}

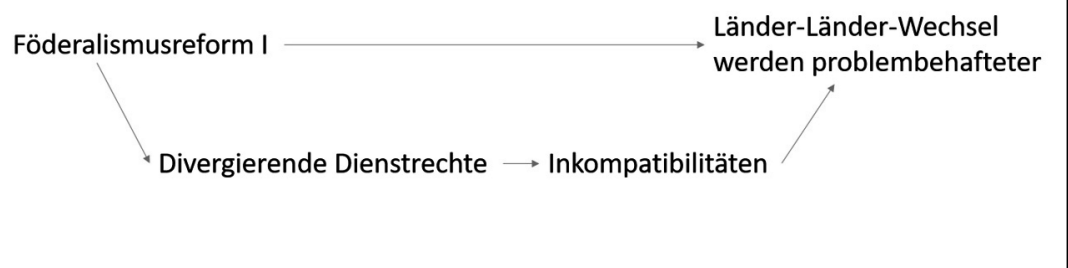

Quelle: Eigene Darstellung.

Einerseits wird dabei Hypothesen testend vorgegangen, da Erkenntnisse aus der Vorschriftenanalyse (Burmester 2015) aufgegriffen werden. Andererseits wird mit der explorativen Rekonstruktion der Fälle auch Hypothesen generierend gearbeitet. Die Analyse erfolgte dabei stets mit Fokus auf den Vorgang des Wechsels und die damit verbundenen Probleme. Die Kategorisierung der Probleme erfolgte unter Rückgriff auf eine an Mayrings (2010) Vorstellungen angelehnte qualitative Inhaltsanalyse. Um die Aspekte, die einem Bundesländergrenzen übergreifenden Dienstherrnwechsel förderlich sind, herausarbeiten zu können, wurde mit einem an das "pattern matching" nach Yin (2013) angelehntes Verfahren gearbeitet. In Kapitel 6 werden die entsprechenden Erkenntnisprozesse jedoch nicht detailliert nachgezeichnet, vielmehr steht eine konzentrierte Darlegung der Ergebnisse im Vordergrund.

Insgesamt wurden 32 nicht-repräsentative Fallstudien mit Blick auf den Wechsel von Beamten der allgemeinen Verwaltung, der Polizei und des Lehramts durchgeführt. Dabei wurden sechs Interviews mit Beamten der allgemeinen Verwaltung, 20 Interviews mit Polizeibeamten und sechs Interviews mit verbeamteten Lehrern geführt. Jenseits der konkreten Fallstudien wurde zur weiteren Validierung der Ergebnisse mit einem Vertreter einer Polizeigewerkschaft und einem Vertreter einer Lehrergewerkschaft gesprochen sowie mit zwei Beamten, die personalpolitisch aktiv sind.

Die Interviews wurden auf der Basis eines halbstandardisierten Fragebogens geführt, welcher analytisch aus mehreren Teilen besteht. So ist der Fragebogen zunächst auf die Erfassung der Motivation für den Wechsel gerichtet. Auch galt es, die Informationssuche des Beamten über das Verfahren und die Komplexität des Wechsels systematisch zu dokumentieren. Anschließende Fragen sind auf die Rekonstruktion des Ablaufs des jeweils be- 
trachteten Wechsels gerichtet. Dabei wurden Handlungsaktionen des Beamten erhoben und diese auch in einen zeitlichen Kontext eingebettet. Somit kann ein Fall jederzeit nicht nur sachlich, sondern auch zeitlich nachvollzogen werden. Es folgen stark standardisierte Fragen. Mit ihnen wird auf die Vorschriftenanalyse und die dort herausgearbeiteten Probleme rekurriert (siehe Burmester 2015 sowie Kapitel 4). Der abschließende Teil des Fragebogens ist offen und explorativ angelegt, um weitere, über die bisherigen Erkenntnisse hinausgehende Probleme, deren Ursachen und Wirkungen sowie den Umgang mit ihnen zu erfassen. Insgesamt umfasst der Fragebogen 104 Items.

Der standardisierte Teil ist auf die jeweilige Beamtengruppe angepasst, da bei unterschiedlichen Gruppen je spezifische Probleme auftreten können. So gibt es beispielsweise bei Lehrkräften in der Sekundarstufe II keine Regelbeförderungen, wie man dies bei Polizeibeamten kennt. Eine Beförderung in eine höhere Besoldungsgruppe erfolgt mit Ausnahme von Bayern in der Regel nur bei der Übernahme einer Funktionsstelle. Folglich entfallen Fragen zu Mindestdienstzeiten bei Beförderungen. Dafür werden bei Lehrern beispielsweise Probleme hinsichtlich der Fächerkombination oder mit dem studierten Lehramtstyp erfragt. Solche inhaltlichen Schwerpunkte der Ausbildung sind bei anderen Beamtengruppen bedeutungslos. In einem vorletzten Schritt wird die Berufsbiographie detailliert aufgenommen. Der Fragebogen schließt mit den soziobiographischen Angaben. 


\section{Besoldungsunterschiede im Ländervergleich: potenzieller Anreiz für einen Wechsel des Bundeslandes}

Die Besoldung ist ein relativ neutrales Maß zur Beurteilung der Anreize oder Hürden eines Bundeslandwechsels. Andere Bereiche des Dienstrechts sind hier deutlich subjektiver. So kann beispielsweise die Absicherung des Beamten im Krankheitsfall durch den Dienstherrn unterschiedlich ausgestaltet sein. Die konkrete Ausgestaltung ist dabei jedoch nicht eindeutig als Anreiz oder Hürde benennbar, sondern die Einordnung erfolgt erst durch die individuelle Wahrnehmung des Beamten. So kann der Gesetzgeber die Absicherung durch ein Beihilfesystem, durch eine Heilfürsorge, eine Versicherung in der gesetzlichen Krankversicherung oder auch über einen kostenfreien Zugang zur ärztlichen Versorgung sicherstellen. Bei der Ausgestaltung beschränken den Gesetzgeber lediglich das Alimentationsprinzip und die Fürsorgepflicht aus Art. 33 Abs. 5 GG. Das heißt, die finanzielle Absicherung muss so ausgestaltet sein, dass diese auch im Fall von Krankheit und den damit verbundenen Mehrkosten amtsangemessen ist (siehe Wichmann/ Langer 2017: 458). Ob die Ausgestaltung nun jedoch als Anreiz oder Hürde wahrgenommen wird, ist individuell unterschiedlich. So kann beispielsweise das Beihilfesystem für kinderlose Beamte als Anreiz wirken, da sie sich einerseits günstig versichern können und andererseits beim Arztbesuch als Privatpatient bessere Leistungen abrufen können. Dahingegen kann beispielsweise für einen Beamten mit mehreren Kindern eine Krankenkassenlösung, bei der alle Familienmitglieder automatisch mitversichert sind, deutlich günstiger sein, sodass bei diesem Beamten diese Ausgestaltung eher als Anreiz wirkt.

Gerade diese subjektive Wahrnehmung ist bei der Besoldung nicht erkennbar. Zwar ist es denkbar, dass andere Bereiche des Dienstrechts vor dem Hintergrund von Hürden und Anreizen die Besoldung überlagern und bei der Entscheidung für oder gegen ein anderes Bundesland eine höhere Priorität einnehmen. Dennoch ist ein Fall nur schwer vorstellbar, bei dem der Beamte ein niedrigeres Salär generell als erstrebenswert erachtet.

Um mit einer allumfassenden Darstellung der Besoldung nicht in Unübersichtlichkeit zu verfallen, werden hier lediglich die Besoldungsgruppen A 7, A 9 und A 13 exemplarisch untersucht. Dabei sind die mit der Be- 
soldungsgruppe verbundenen Amtsbezeichnungen ${ }^{7}$ und Laufbahngruppen aufgrund der Varianz im Laufbahnrecht über die Länder hinweg nicht immer identisch. Dennoch soll eine Einordnung am Laufbahnrecht von Baden-Württemberg exemplarisch erfolgen. Dabei kann das Ergebnis zumindest im Grundsatz auf die anderen Länder mit anderen Systemen übertragen werden, auch wenn die Begrifflichkeiten sich häufig deutlich unterscheiden (vgl. Burmester 2015, S. 34 f.).

Die Besoldungsgruppe A 7 entspricht einer Besoldungsgruppe des mittleren Dienstes und wird beispielsweise mit der Amtsbezeichnung Polizeimeister oder Brandmeister als Eingangsamt verliehen. Die Besoldungsgruppe A 9 entspricht dem Verzahnungsamt mittlerer Dienst/gehobener Dienst und wird beispielsweise einem Polizeihauptmeister im Endamt des mittleren Dienstes oder einem Polizeikommissar als Eingangsamt des gehobenen Dienstes verliehen. Die Besoldungsgruppe A 13 entspricht dem Verzahnungsamt des gehobenen/höheren Dienstes. Sie wird dem Ersten Polizeihauptkommissar als Endamt des gehobenen Dienstes oder im höheren Dienst als Eingangsamt des Studienrats für Gymnasien oder berufsbegleitende Schulen verliehen (vgl. $\$ 14$ LBG BaWü; vgl. Anlage 1 LBesGBW).

Für die Berechnung wird das Bruttojahresgrundgehalt in der Endstufe der Besoldungstabelle, zusätzlich allgemeiner Stellen- oder Strukturzulagen sowie etwaiger einmaliger Jahressonderzahlungen herangezogen. Da die Arbeitszeiten zwischen den Bundesländern variieren, ist für eine bessere Vergleichbarkeit die Besoldung rechnerisch auf eine 40h-Woche standardisiert worden. So gelten in fast allen Ländern 40 Stunden als allgemeine Wochenarbeitszeit für Beamte. Eine Ausnahme bilden lediglich Baden-Württemberg, Hessen, Nordrhein-Westfalen und Schleswig-Holstein, welche eine allgemeine Wochenarbeitszeit von 41 Stunden aufweisen.

Die Gründe für die Varianz in der Besoldung sind vielfältig. So konnten Dose und Wolfes für die Besoldung im Jahre 2014 zeigen, dass der Verschuldungsdruck eine dominante erklärende Variable für die Besoldungshöhe darstellt. Ebenso ist die Mitgliedschaft in der Tarifgemeinschaft deutscher Länder (TdL) als Variable fast mit gleicher Stärke wie der Verschuldungsdruck erklärungskräftig. Zwar ist das ausgehandelte Ergebnis einer Tarifrunde zwischen Gewerkschaften und Arbeitgebern des öffentlichen Dienstes prinzipiell nur auf die Tarifangestellten anzuwenden, es zeigt sich aber, dass ein einmal verhandeltes Ergebnis als Ankerpunkt bei Besoldungsanpassungen einen hohen Druck erzeugt. Ebenso zeigten die Autoren, dass die An-

7 Nicht nur die Amtsbezeichnungen unterscheiden sich, teilweise variieren auch die mit der Besoldungsgruppe verbundenen Ämter. So existiert beispielsweise der Polizeioberwachtmeister als Amt mit der Besoldung nach A5 ausschließlich in Bayern. 
zahl der Vetospieler und die parteipolitische Präferenz des Ministerpräsidenten erklärungskräftig sind. Die Stärke dieses Einflusses ist jedoch deutlich geringer als die des Verschuldungsdrucks und die einer Mitgliedschaft in der Tarifgemeinschaft deutscher Länder. Dennoch ist eine desto höhere Besoldung zu erwarten, umso niedrigerer die Anzahl der Koalitionspartner, also der Vetospieler innerhalb der Regierung ist. Auch besolden Bundesländer mit konservativen Ministerpräsidenten im Schnitt höher als Bundesländer mit linkem Ministerpräsidenten (siehe Dose/Wolfes 2016, S. 286 ff.).

Abbildung 2: A 7-Jahresbruttobesoldung der Beamten der Länder und Kommunen bei Annahme einer 40-Stunden-Woche im Vergleich der Bundesländer im Jahr 2019

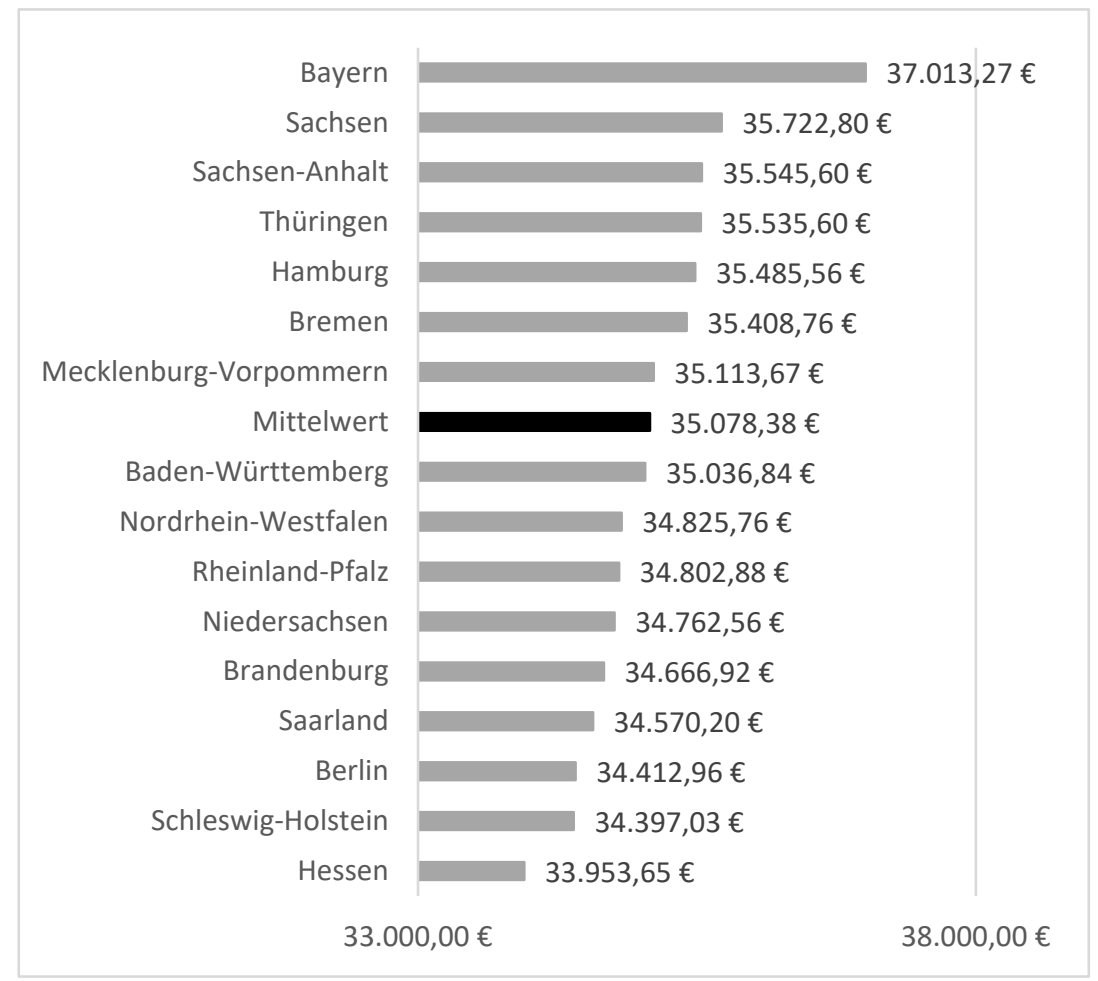

Datenquelle: DGB Bundesvorstand 2019, S. 18. 
Abbildung 2 zeigt die Verteilung einer Besoldung nach A 7 zwischen den Bundesländern. Der Durchschnitt aller Bundesländer liegt bei 35.078,38 € mit einer Standardabweichung von 673,16. Insgesamt befinden sich sieben Bundesländer mit ihrer Besoldung oberhalb des Durchschnittes (Bayern, Sachsen, Sachsen-Anhalt, Thüringen, Hamburg, Bremen und MecklenburgVorpommern) und neun Bundesländer unterhalb des Durchschnittes (Baden-Württemberg, Nordrhein-Westfalen, Rheinland-Pfalz, Niedersachsen, Brandenburg, Saarland, Berlin, Schleswig-Holstein und Hessen). Auffällig ist hierbei, dass Bayern deutlich höher besoldet als alle anderen Bundesländer. Der Abstand zwischen Bayern als dem am höchsten besoldenden Bundesland $(37.013,27 €)$ und Sachsen, dem Bundesland mit der zweithöchsten Besoldung (35.722,80 €), beträgt immerhin bereits 1.290,47 €. Ein ähnlicher Ausreißer, wenn auch nicht in dieser Extremform, ist am unteren Ende der Verteilung zu finden. Der Abstand zwischen Hessen, dem Bundesland mit der niedrigsten Besoldung (33.953,65 €) und Schleswig-Holstein, als Bundesland mit der zweitniedrigsten Besoldung in dieser Besoldungsgruppe, beträgt 443,38 €. Im Vergleich dazu: Der durchschnittliche Abstand zwischen allen Bundesländern beträgt lediglich 203,97 €. Ohne die Ausreißer (also ohne Bayern und Hessen) halbiert sich der durchschnittliche Besoldungsunterscheid von Land zu Land sogar noch auf 101,98 €.

Der Abstand zwischen dem am höchsten besoldenden Bundesland, Bayern, und dem am niedrigsten besoldenden Bundesland, Hessen, liegt bei 3.059,62 €. Dies entspräche bei einem Wechsel von Hessen nach Bayern einer Besoldungserhöhung von 9 Prozent oder im umgekehrten Fall, eine Besoldungsverringerung von 8,1 Prozent. Auch hieran ist die Stärke der Ausreißer im Verhältnis erkennbar. Allein 56,7 Prozent der Spannweite machen die Abstände der beiden Bundesländer Bayern und Hessen zu ihren direkten Nachbarn (Sachsen für Bayern und Schleswig-Holstein für Hessen) in der Verteilung aus. Auf alle übrigen vierzehn Bundesländer verteilen sich die übrigen 44,3 Prozent.

Die Verteilung der Besoldung nach A 9 ist in der Abbildung 3 dargestellt. 
Abbildung 3: A 9-Jahresbruttobesoldung der Beamten der Länder und Kommunen bei Annahme einer 40-Stunden-Woche im Vergleich der Bundesländer im Jahr 2019

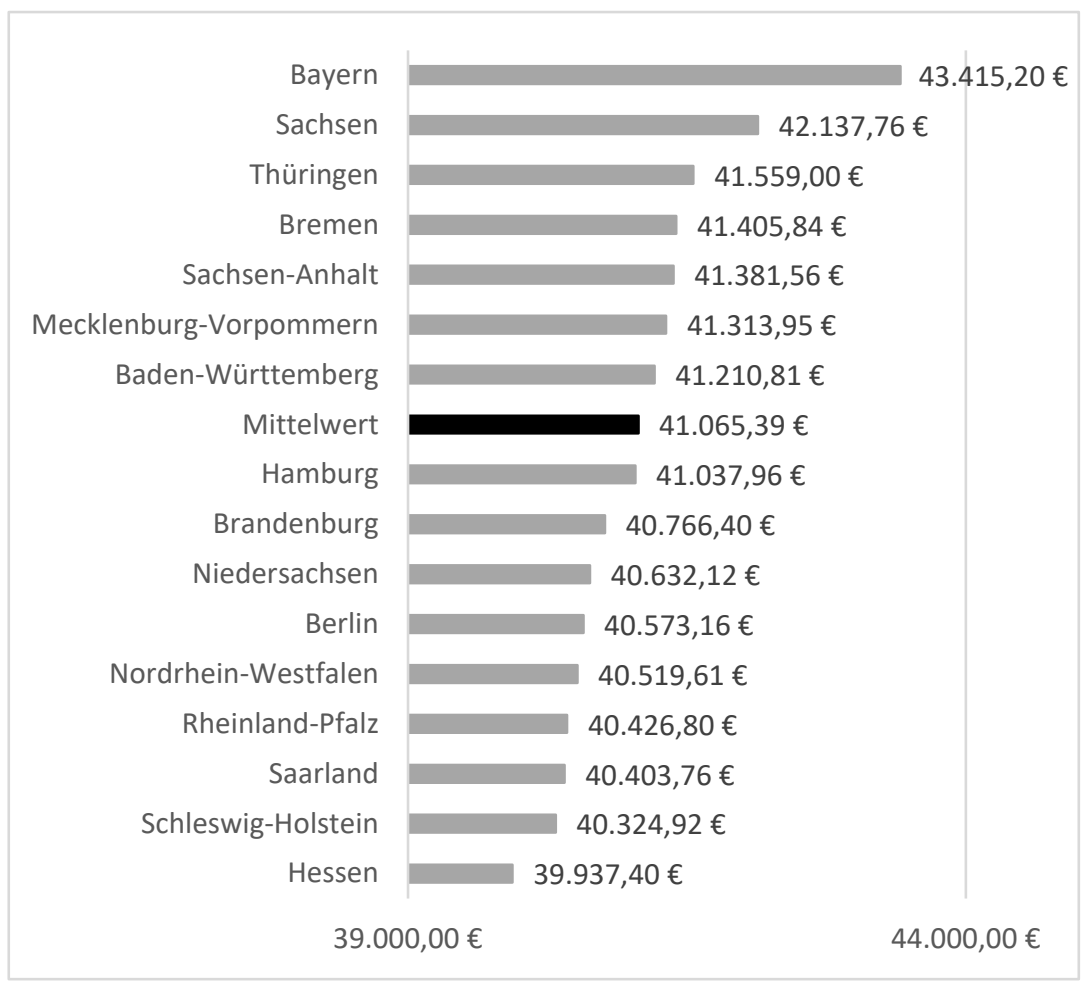

Datenquelle: DGB Bundesvorstand 2019, S. 20.

Der Durschnitt der Besoldungsgruppe liegt bei 41.065,39€ mit einer Standardabweichung von 817,71. Auch hier liegen sieben Bundesländer über dem Durchschnitt (Bayern, Sachsen, Thüringen, Bremen, Sachsen-Anhalt, Mecklenburg-Vorpommern, Baden-Württemberg) und neun befinden sich darunter (Hamburg, Brandenburg, Niedersachsen, Berlin, Nordrhein-Westfalen, Rheinland-Pfalz, Saarland, Schleswig-Holstein, Hessen).

Während bei der Besoldung nach A 7 Hamburg noch oberhalb des Durschnittes besoldet, liegt das Bundesland nun knapp unterhalb des Dur- 
schnittes. Die Besoldung Baden-Württembergs hingegen, in der Verteilung nach A 7 noch leicht unterdurchschnittlich, liegt hier nun knapp oberhalb des Durchschnittes. Oberhalb des Durchschnittes fällt Saschen-Anhalt in der Verteilung ab und steht nunmehr auf Platz fünf (A 7: Platz 3), während Bremen sich gegenüber der A 7-Besoldung leicht verbessert und sich nun auf Platz vier befindet (A 7: Platz 6). Auffällig bei den Bundesländern unterhalb des Durchschnittes ist, dass Rheinland-Pfalz und Nordrhein-Westfalen, welche bei der Besoldung nach A 7 die Plätze 11 und 10 der Verteilung belegen, nun nur noch Platz 15 und 14 belegen. Berlin und Brandenburg steigen in der Verteilung hingegen weiter auf und belegen die Plätze 11 (A 7: Platz 14) und 9 (A 7: Patz 12).

Wie bei der Besoldung nach A 7 ist auch bei der nach A9 ein Muster mit Ausreißern am oberen und unteren Ende der Besoldungsverteilung zu erkennen. Bayern führt auch hier die Besoldungsverteilung mit 43.415,20€ an, gefolgt von Sachsen mit 42.137,76€. Der Abstand zwischen diesen beiden Bundesländern beträgt 1.277,44 €. Wie bei der Besoldung nach A 7 befindet sich gleichfalls ein Ausreißer am unteren Ende der Verteilung. Auch hier ist Hessen das Bundesland mit der niedrigsten Besoldung (39.937,40€). Der Abstand zwischen Hessen und Schleswig-Holstein, welches das Bundesland mit der zweitniedrigsten Besoldung ist, beträgt 387,92 €. Einen Unterschied zur Verteilung nach A 7 ist jedoch erkennbar. Wo vorher nur Bayern und Hessen die Ausreißer darstellten, kommt bei der Besoldungsgruppe A 9 Sachsen als dritter Ausreißer mit einem Abstand von 578,76€ zu Thüringen, dem Bundesland mit der dritthöchsten Besoldung, hinzu. Neben der grafischen Auffälligkeit in der Abbildung 3 kann diese Feststellung auch mit einem Vergleich zum Wert der durchschnittlichen Besoldungserhöhung untermauert werden. Der durchschnittliche Besoldungsabstand zwischen den Bundesländern liegt bei 231,85€. Die Ausreißer liegen in diesem Fall mit $1.277,44 €$ (Bayern-Sachsen), 578,76€ (Sachsen-Thüringen) und 287,92 € (Hessen-Schleswig-Holstein) teils deutlich darüber. Mit Ausnahme des Abstandes zwischen Brandenburg und Hamburg, welcher mit 271,56€ auch leicht über dem durchschnittlichen Besoldungsabstand der Verteilung liegt, liegen alle übrigen Bundesländer mit einem Abstand zu ihren direkten Nachbarn in der Verteilung unter dem Durchschnitt von 231,85€. Und auch der Unterschied zwischen dem durchschnittlichen Besoldungsunterschied mit und ohne Ausreißer lässt die Stärke der Abweichung erkennen. Wenn mit den Ausreißern dieser Wert noch bei 231,85 € liegt, halbiert sich dieser ohne die drei Ausreißer auf 102,84 €. Vergleicht man diese Werte mit denen der Besoldung nach A 7, zeigt sich, dass die Werte ohne Ausreißer zwischen A 7 und A 9 sehr ähnlich sind. Mit den Ausreißern steigt dieser 
Wert jedoch bei der Besoldung nach A 9 im Vergleich zur Besoldung nach A 7 an.

Die Spannweite zwischen Bayern, dem am höchsten besoldenden Bundesland, und Hessen, dem Bundesland mit der niedrigsten Besoldung, liegt bei 3.477,80 €, was bei einem Wechsel zwischen diesen beiden Bundesländern eine Besoldungserhöhung von 8,7 Prozent bzw. eine Besoldungsverringerung von 8 Prozent zur Folge hätte. Dabei können die drei Ausreißer insgesamt 64,5 Prozent der Spannweite erklären.

Abbildung 4 stellt die Verteilung der Besoldung nach A 13 dar. Die Durchschnittliche Besoldung liegt bei 61.784,24 € mit einer Standardabweichung von 1.394,93. Es liegen auch hier sieben Bundesländer (Bayern, Sachsen, Baden-Württemberg, Sachsen-Anhalt, Thüringen, Mecklenburg-Vorpommern, Niedersachsen) über und neun Bundesländer unter dem Mittelwert der Verteilung (Hamburg, Brandenburg, Bremen, Hessen, NordrheinWestfalen, Saarland, Berlin, Rheinland-Pfalz, Schleswig-Holstein).

Im Hinblick auf die Position der einzelnen Bundesländer in der Verteilung sind vor allem Niedersachsen, Hessen, Berlin, Rheinland-Pfalz, BadenWürttemberg und Bremen interessant. Während Niedersachsen bei seiner Besoldung nach A 7 und A 9 noch unterhalb des Durchschnittes rangierte, befindet es sich mit seiner Besoldung nach A 13 nun knapp oberhalb des Durchschnittes. Hessen, bei A 7 und A 9 jeweils das Bundesland mit der niedrigsten Besoldung, liegt nun nur noch auf Platz 11, womit nun Schleswig-Holstein das Ende der Verteilung darstellt. Während Berlin bei seiner A 7-Besoldung noch den drittletzten Platz in der Verteilung belegt, steigt das Bundesland bei seiner A 9-Besoldung in der Verteilung auf den 11. Platz, während es nach A 13 wieder auf den drittletzten Platz zurückfällt. Rheinland-Pfalz sinkt in der Verteilung weiter ab und kommt mit seiner A 13-Besodung nur noch auf den vorletzten Platz (A 7: Platz 10; A 9: Platz 13). Baden-Württemberg hingegen, nach A 7 noch knapp unterdurchschnittlich und nach A 9 knapp überdurchschnittlich besoldend, setzt den aufsteigenden Trend fort und belegt mit seiner A 13-Besoldung den dritthöchsten Platz, nach Bayern und Sachsen. Bremen, bei A 7 und A 9 noch überdurchschnittlich besoldend, befindet sich mit seiner A 13-Besoldung nur noch unterhalb des Durchschnittes. 
Abbildung 4: A 13-Jahresbruttobesoldung der Beamten der Länder und Kommunen bei Annahme einer 40-Stunden-Woche im Vergleich der Bundesländer im Jahr 2019

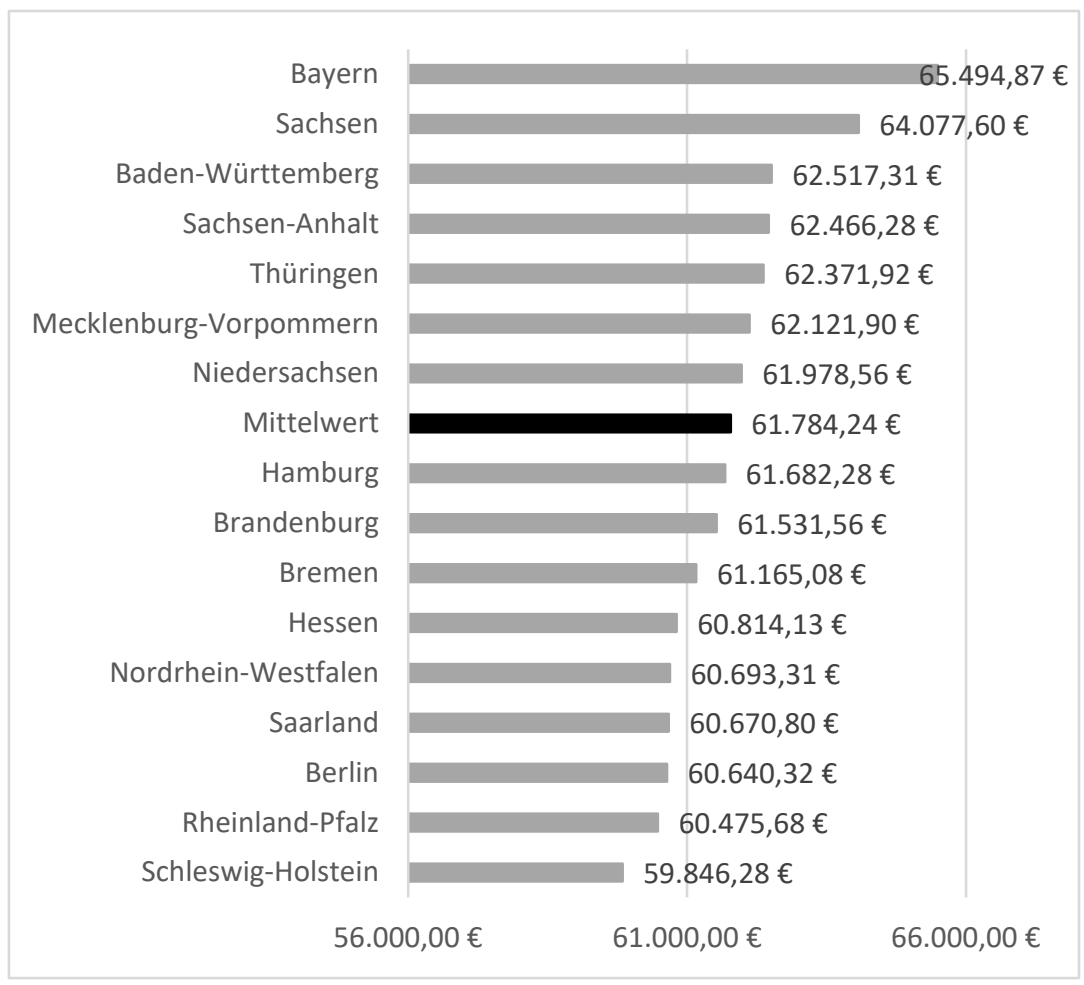

Datenquelle: DGB Bundesvorstand 2019, S. 22.

Das Muster gleicht der Verteilung der Besoldung nach A 9. So existieren an der Spitze der Verteilung zwei Bundesländer und am Ende der Verteilung ein Bundesland, welches sich mit der Höhe seiner A 13-Besoldung deutlich von den anderen Bundesländern in der Verteilung abhebt. Dabei steht Bayern mit 65.494,87€ an erster Stelle der Verteilung. An zweiter Stelle steht mit einem Abstand von 1.417,27 € auch hier wieder Sachsen (64.494,87 €). Der Abstand zwischen Sachsen und dem drittplatzierten Baden-Württemberg liegt bei 1.560,29 €. Ebenso ist der Abstand zwischen dem 
letzten Bundesland in der Tabelle (Hessen: 59.846,28 €) und dem vorletzten Bundesland (Rheinland-Pfalz: 60.475,68 €) mit 629,40€ relativ hoch. Im Vergleich dazu: Der durchschnittliche Besoldungsunterschied liegt bei 376,57 €. Auch hier ist der Abstand aller weiteren Bundesländer relativ harmonisch und liegt ohne die Ausreißer durchschnittlich bei 170,14 €.

Die Spannweite der Verteilung liegt bei 5.648,59 €, was beutet, dass ein Wechsel zwischen Bayern und Schleswig-Holstein eine Besoldungserhöhung von 9,4 Prozent oder eine Besoldungsverringerung von 8,6 Prozent zur Folge hätte. Dabei liegt der Anteil der Ausreißer an dieser Spannweite bei 63,9 Prozent und damit ähnlich hoch, wie bei der Besoldung nach A 9.

Im Vergleich über alle drei Verteilungen hinweg kann festgestellt werden, dass ein Wettbewerb zwischen den Bundesländern vorhanden ist, da der Abstand zwischen den beiden Extrempunkten bei allen untersuchten Besoldungsgruppen bei knapp 10 Prozent liegt. Es lässt sich aber auch feststellen, dass der Wettbewerb vor allem von einigen Ausreißern angetrieben wird, was der Vergleich des durchschnittlichen Besoldungsabstandes mit und ohne Ausreißer zeigt sowie der Anteil dieser Bundesländer an der Spannweite. Außerdem deutet sich an, dass mit den höheren Besoldungsgruppen auch der Wettbewerb zunimmt. Dafür gibt es zwei Hinweise: Erstens nimmt die Standardabweichung mit der Höhe der Besoldungsgruppe zu, was zeigt, dass die Streuung um den Mittelwert zunimmt. Zweitens kommt mit Sachsen ab der Besoldung nach A 9 ein weiteres Bundesland hinzu, dessen Besoldungsabstand zu seinen Nachbarn in der Verteilung deutlich über dem durchschnittlichen Besoldungsabstand liegt. 


\section{Vorschriftenanalyse}

Die nachfolgend präsentierte Analyse der Vorschriften ${ }^{8}$ soll Aufschluss darüber geben, inwieweit sich die Laufbahnrechte der Bundesländer auseinanderentwickelt haben und welche möglichen Konsequenzen sich daraus für die Bundesländer übergreifende Mobilität von Beamten ergeben. Im Fokus der Betrachtung stehen Polizisten, Lehrer und Beamte der allgemeinen Verwaltung. Diese Beamtengruppen wurden für die Analyse ausgewählt, da bei Lehrern und Polizisten bereits vor der Föderalismusreform I eine starke Föderalisierung vorlag und diese somit als Referenzgruppen zu den Beamten der allgemeinen Verwaltung herangezogen werden können. Berücksichtigt ist der Stand der Gesetze und Verordnungen bis Mai 2015. Das hier präsentierte Kapitel ist eine Kurzfassung der Vorschriftenanalyse, welche im Jahr 2015 in der Reihe der Duisburger politik- und verwaltungswissenschaftlichen Arbeitspapiere veröffentlicht wurde (Burmester 2015). Im Vergleich zu der zuvor veröffentlichten Analyse werden hier die Bereiche in den Fokus genommen, die sich im Verlauf unserer Forschungen als tatsächlich mobilitätshemmend erwiesen haben.

Von einem attraktiven Laufbahnrecht, das etwa durch beamtenfreundliche Aufstiegs- und Weiterbildungsmöglichkeiten gekennzeichnet ist, kann ein Mobilitätsanreiz ausgehen. Eine allzu stark ausgeprägte Fragmentierung kann jedoch auch dazu führen, dass die Laufbahnsysteme miteinander inkompatibel werden, eine Anerkennung von Ausbildung, Vorbereitungsdienst und Weiterqualifizierungen nicht gewährleistet ist und somit Mobilitätshemmnisse aufgebaut werden. Diese letztgenannten Auswirkungen können dem eigentlichen Hintergrund der Novellierung des Laufbahnrechts, nämlich den öffentlichen Dienst in Reaktion auf die Herausforderungen des demographischen Wandels attraktiver zu gestalten (vgl. Idecke-Lux 2014, S. 113), entgegenstehen.

Nachfolgend soll der Fragmentierungsgrad der Laufbahngesetze im Ländervergleich analysiert und mögliche Auswirkungen diskutiert werden. In Kapitel 4.1 wird auf die mittlerweile recht unterschiedlichen Laufbahnsysteme eingegangen. Sie sind von einer sowohl unterschiedlichen Zahl an Laufbahngruppen als auch an Fachlaufbahnen gekennzeichnet. Nach dieser grundlegenden Darlegung wird in einer jeweils beamtengruppenspezifischen Betrachtung sowohl auf die Mobilitätsanreize als auch auf die Mobilitätshemmnisse eingegangen. In Kapitel 4.2 wird dabei der Blick auf die all-

8 Dieses Kapitel baut auf Burmester 2015 auf. 
gemeine Verwaltung, in Kapitel 4.3 auf die Polizei und in Kapitel 4.4 auf den Lehramtsbereich gerichtet. Im das dieses Hauptkapitel abschließenden Zwischenfazit (Kapitel 4.5) werden auf Basis der erfolgten Vorschriftenanalyse einige Thesen und Hypothesen formuliert, welche den weiteren Forschungsprozess anleiten und strukturieren sollen.

\subsection{Die Neuregelung der Laufbahnsysteme}

Tabelle 1 gibt einen Überblick über die neuen Laufbahnsysteme der Bundesländer. Während in Brandenburg und dem Saarland noch das klassische viergliedrige Laufbahnsystem mit einfachem bis höherem Dienst besteht, wurde in Thüringen, Hessen und Baden-Württemberg der einfache Dienst komplett abgeschafft. Die norddeutschen Küstenländer sowie Berlin, Sachsen, Sachsen-Anhalt und zuletzt auch Baden-Württemberg haben eine erste und zweite Laufbahngruppe mit jeweils einem ersten und zweiten Einstiegsamt eingeführt. Bayern und Rheinland-Pfalz hingegen weisen nur noch eine Leistungs- bzw. Einheitslaufbahn mit vier Einstiegsämtern bzw. Qualifikationsebenen auf. Grundsätzlich lässt sich aber in allen Bundesländern wegen der Schaffung verschiedener Eingangsämter noch eine Orientierung an dem viergliedrigen einfachen bis höheren Dienst erkennen. Ergänzend sei angemerkt, dass auch im täglichen Sprachgebrauch der Beamten noch immer vorwiegend mit den Begriffen des einfachen, mittleren, gehobenen und höheren Dienstes gearbeitet wird (vgl. Baßlsperger 2012, S. 400), weshalb dies zur sprachlichen Vereinfachung auch im Laufe der Arbeit getan wird.

Die Anforderungen zur Einstellung in den Vorbereitungsdienst waren vor der Föderalismusreform in $\$ 13 \mathrm{BRRG}^{9}$ geregelt und sind nun in den Landesbeamtengesetzen verankert. Grundsätzlich sind die Anforderungen an die formale Qualifikation für die Einstellung in den Vorbereitungsdienst jedoch nicht so fragmentiert, dass dies zu nachhaltigen Problemen bei einem angestrebten Bundesländergrenzen überschreitenden Wechsel kommen sollte.

9 Rahmengesetz zur Vereinheitlichung des Beamtenrechts (Beamtenrechtsrahmengesetz, BRRG) in der Fassung vom 31. März 1999, BGBL I Nr. 18, ausgegeben am 14.04.1999, S. 654-674. 
Tabelle 1: Die neuen Laufbahnsysteme der Bundesländer

\begin{tabular}{|c|c|c|c|c|c|c|c|}
\hline \multicolumn{2}{|l|}{$\begin{array}{l}\text { BRANDENBURG, } \\
\text { NORDRHEIN- } \\
\text { WESTFALEN, } \\
\text { SAARLAND }\end{array}$} & $\begin{array}{l}\text { Thüringen, He } \\
\text { Baden-Württen }\end{array}$ & EEN, & \multicolumn{2}{|c|}{$\begin{array}{l}\text { NoRdDEUTSCHE } \\
\text { KüSTENLÄNDER + } \\
\text { BERLIN, SACHSEN, } \\
\text { SACHSEN-ANHALT }\end{array}$} & \multicolumn{2}{|c|}{$\begin{array}{c}\text { Bayern UND } \\
\text { RhEINLAND-PFALZ }\end{array}$} \\
\hline $\begin{array}{l}\text { EINFACHER } \\
\text { DIENST }\end{array}$ & EA & & & \multirow{2}{*}{$\begin{array}{l}\text { 1. Lauf- } \\
\text { bahn- } \\
\text { gruppe }\end{array}$} & 1. Einstiegsamt & \multirow{4}{*}{$\begin{array}{c}\text { (Leis- } \\
\text { tungs-) } \\
\text { Laufbahn }\end{array}$} & $\begin{array}{l}\text { 1. EA/ } \\
\text { Qualifi- } \\
\text { kations- } \\
\text { ebene }\end{array}$ \\
\hline $\begin{array}{l}\text { Mittlerer } \\
\text { Dienst }\end{array}$ & EA & $\begin{array}{c}\text { Mittlerer } \\
\text { Dienst }\end{array}$ & EA & & 2. Einstiegsamt & & $\begin{array}{l}\text { 2. EA/ } \\
\text { Qualifi- } \\
\text { kations- } \\
\text { ebene }\end{array}$ \\
\hline $\begin{array}{c}\text { Gehobener } \\
\text { Dienst }\end{array}$ & EA & $\begin{array}{l}\text { Gehobener } \\
\text { Dienst }\end{array}$ & EA & \multirow{2}{*}{$\begin{array}{l}\text { 2. Lauf- } \\
\text { bahn- } \\
\text { gruppe }\end{array}$} & 1. Einstiegsamt & & $\begin{array}{l}\text { 3. EA/ } \\
\text { Qualifi- } \\
\text { kations- } \\
\text { ebene }\end{array}$ \\
\hline $\begin{array}{l}\text { HÖHERER } \\
\text { DiENST }\end{array}$ & EA & Höherer Dienst & EA & & 2. Einstiegsamt & & $\begin{array}{l}\text { 4. EA/ } \\
\text { Qualifi- } \\
\text { kations- } \\
\text { ebene }\end{array}$ \\
\hline
\end{tabular}

$\mathrm{EA}=$ Einstiegsamt

Quelle: Burmester 2015, S.36.

Über die Reformierung der Laufbahnsysteme hinaus wurden auch die Fachlaufbahnen reduziert. Während vor der Föderalismusreform I noch über einhundert Fachlaufbahnen bestanden, wurde die Anzahl je nach Bundesland auf sechs bis elf reduziert. Ausnahmen bilden Baden-Württemberg, Brandenburg und Nordrhein-Westfalen (vgl. Burmester 2015, S. 35f).

\subsection{Mobilitätsanreize und -hemmnisse für Beamte der allgemeinen Verwaltung}

Bei der allgemeinen Verwaltung haben sich vor allem die Aufstiegs- und Beförderungsmöglichkeiten im Vergleich der Bundesländer ausdifferenziert. Vor der Föderalismusreform wurden diese im BRRG geregelt und boten den Bundesländern nur wenig Spielraum für Abweichungen. Dies gilt gleichermaßen für die Dauer des Vorbereitungsdienstes und die Anforderungen an den Erwerb der Laufbahnbefähigung. Wurde ein solcher Spielraum gewährt, wurde er jedoch kaum von der jeweiligen Landesgesetzgebung genutzt, sodass sich durch die geringe Ausdifferenzierung keine Nachteile für die Beamten ergaben. Für den Fall einer heterogenen Ausgestaltung und 
einem Bundeslandwechsel sah $₫ 122$ Abs. 2 BRRG vorbeugend vor: „Wer unter den Voraussetzungen der $\mathbb{S} 13$ bis $14 \mathrm{c}$ die Befähigung für eine Laufbahn erworben hat, besitzt die Befähigung für entsprechende Laufbahnen bei allen Dienstherren im Geltungsbereich dieses Gesetzes“.

Die Mobilitätshemmnisse und -anreize werden für die Zeit vor der Föderalismusreform folglich als sehr gering eingeschätzt. Weder ergaben sich aus der nur in sehr geringem Ausmaß vorhandenen heterogenen Ausgestaltung der Laufbahnverordnungen der Länder Mobilitätshemmnisse, noch wird von diesen ein Anreiz für einen Bundeslandwechsel ausgegangen sein (vgl. Burmester 2015, S. 19).

Nach der Föderalismusreform I nutzten die Länder ihre neuen Kompetenzen vor allem für eine Neugestaltung der Aufstiegs- und Beförderungsmöglichkeiten. Tabelle 2 bietet einen Überblick über die vorgesehenen Aufstiege nach Bundesländern ${ }^{10}$. Der Regelaufstieg wird weiterhin von allen Bundesländern angeboten, jedoch variieren die Mindestdienstzeiten je nach Bundesland und Laufbahngruppe zwischen ein und zehn Jahren (ein Jahr z.B. in Brandenburg vom einfachen in den mittleren Dienst, zehn Jahre z.B. in Brandenburg und Saarland vom gehobenen in den höheren Dienst). Ein Regelaufstieg vom einfachen in den mittleren Dienst wird in den meisten Bundesländern nicht mehr angeboten. In Bayern, Hessen und NordrheinWestfalen ist der Regelaufstieg vom gehobenen in den höheren Dienst zum Beispiel nicht mehr möglich (Kathke 2011, S.60; Schrapper 2016, S. 401). Idecke-Lux begründet dies wie folgt:

„Der Aufstieg vom gehobenen in den höheren Dienst, der in Nordrhein-Westfalen auf einem System von "Warten und Beurteilen" basierte, war nicht mehr zeitgemäß. Die längst überfällige Modernisierung des Aufstiegs vom gehobenen in den höheren Dienst greift auch in Nordrhein-Westfalen künftig den Gedanken einer Qualifizierung aus eigenem Bestand auf und sieht nun losgelöst vom Alter, dem Gedanken einer Belohnung für geleistete Dienste und langjährige Dienstzeiten einen qualifizierungsgebundenen Aufstieg entweder durch eine (modulare) Qualifizierung oder durch ein Masterstudium vor" (Idecke-Lux 2014, S. 114f.).

Als Erfolg der Reform kann gelten, dass die Verzahnungsämter in nahezu allen Bundesländern aufgehoben wurden und somit bei einem Aufstieg nicht mehr doppelt durchlaufen werden müssen (vgl. Burmester 2015, S. 39).

10 Abkürzungen in der Tabelle und in den folgenden Tabellen: BW - Baden-Württemberg; BY - Bayern; BE - Berlin; BB - Brandenburg; HB - Freie Hansestadt Bremen; HH - Freie und Hansestadt Hamburg; HE - Hessen; MV - Mecklenburg-Vorpommern; NI - Niedersachsen; NW - Nordrhein-Westfalen; RP - Rheinland-Pfalz; SL - Saarland; SN - Sachsen; ST Sachsen-Anhalt; SH - Schleswig-Holstein; TH - Thüringen. 
Tabelle 2: Vorgesehene Aufstiegsmöglichkeiten im Beamtenrecht der Bundesländer

\begin{tabular}{|c|c|c|c|c|c|c|c|c|c|c|c|c|c|c|c|c|}
\hline & BW & BY & $\mathrm{BE}$ & BB & $\begin{array}{l}\mathrm{H} \\
\mathrm{B}\end{array}$ & $\begin{array}{l}\mathrm{H} \\
\mathrm{H}\end{array}$ & $\begin{array}{l}\mathrm{H} \\
\mathrm{E}\end{array}$ & $\begin{array}{l}\mathrm{M} \\
\mathrm{V}\end{array}$ & $\begin{array}{l}\mathrm{N} \\
\mathrm{I}\end{array}$ & $\begin{array}{l}\mathrm{N} \\
\mathrm{W}\end{array}$ & $\begin{array}{l}\mathrm{R} \\
\mathrm{P}\end{array}$ & & $\begin{array}{l}\mathrm{S} \\
\mathrm{N}\end{array}$ & ST & $\begin{array}{l}S \\
H\end{array}$ & $\begin{array}{l}\mathrm{T} \\
\mathrm{H}\end{array}$ \\
\hline Regelaufstieg & $\mathrm{x}$ & $x^{1}$ & $\mathrm{x}$ & $\mathrm{x}$ & $\mathrm{x}$ & $\mathrm{x}$ & $\mathrm{x}$ & $\mathrm{x}$ & $\mathrm{x}$ & $\mathrm{x}$ & $x^{1}$ & $\mathrm{x}$ & $\mathrm{x}$ & $\mathrm{x}$ & $\mathrm{x}$ & $\mathrm{x}$ \\
\hline $\begin{array}{l}\text { Verwendungs- } \\
\text { aufstieg }\end{array}$ & $\mathrm{x}$ & - & -- & $\mathrm{x}$ & -- & - & - & - & -- & - & - & -- & -- & $\mathrm{x}$ & - & - \\
\hline Praxisaufstieg & -- & - & $\mathrm{x}$ & - & $\mathrm{x}$ & - & $x^{2}$ & $\mathrm{x}$ & $\mathrm{x}$ & - & - & $\mathrm{x}$ & -- & - & -- & $\mathrm{x}$ \\
\hline $\begin{array}{l}\text { Modulare } \\
\text { Qualifizierung }\end{array}$ & -- & $\mathrm{x}$ & -- & -- & -- & -- & -- & -- & -- & $\mathrm{x}$ & $x^{4}$ & -- & -- & -- & -- & -- \\
\hline Studium & $\mathrm{x}$ & - & - & $\mathrm{x}$ & -- & $\mathrm{x}$ & $x^{3}$ & -- & -- & $\mathrm{x}$ & -- & -- & $\mathrm{x}$ & & - & $\mathrm{x}$ \\
\hline $\begin{array}{l}\text { Schnellauf- } \\
\text { stieg }\end{array}$ & -- & - & - & - & -- & - & -- & -- & -- & - & - & -- & -- & - & $\mathrm{x}$ & - \\
\hline $\begin{array}{l}\text { Bewärhungs- } \\
\text { aufstieg }\end{array}$ & -- & - & $\mathrm{x}$ & - & -- & -- & -- & -- & -- & - & -- & -- & -- & - & $\mathrm{x}$ & -- \\
\hline $\begin{array}{l}\text { Erleichterter } \\
\text { Aufstieg }\end{array}$ & - & - & - & - & - & -- & - & $\mathrm{x}$ & -- & - & - & -- & -- & - & - & - \\
\hline
\end{tabular}

--: nicht vorgesehen

$\mathrm{x}$ : vorgesehen

${ }^{1}$ : heißt in Bayern und Rheinland-Pfalz Ausbildungsqualifizierung, ist inhaltlich weitgehend unverändert zum

vorherigen Regelaufstieg

${ }^{2}$ : heißt in Hessen Erfahrungsaufstieg

${ }^{3}$ : heißt in Hessen Qualifikationsaufstieg

${ }^{4}$ : heißt in Rheinland-Pfalz Fortbildungsqualifizierung

Quelle: Burmester 2015, S. 37.

Im Weiteren soll vor allem ein Fokus auf die modulare Qualifizierung gelegt werden, welche augenscheinlich die meisten Hürden aber auch Anreize bei einem Bundeslandwechsel mit sich bringen kann. Diese wird nur in Bayern, Nordrhein-Westfalen und in Rheinland-Pfalz angeboten, wo sie als Fortbildungsqualifizierung bezeichnet wird. Während in Bayern und in Rheinland-Pfalz (mit Ausnahmen) eine Mindestdienstzeit von zehn Jahren für die Teilnahme an einer modularen Qualifizierung notwendig ist, genügt in Nordrhein-Westfalen eine Mindestdienstzeit von zwei Jahren $(\mathbb{\$} 25$ Abs. 1 
LVO NRW ${ }^{11} ; \mathbb{S} 17$ Abs. 6 LlbG) $)^{12}$. Die modulare Qualifizierung dauert in allen genannten Bundesländern nur wenige Wochen und befähigt für den Aufstieg in die nächsthöhere Laufbahngruppe ( $\$ 5$ Abs. 1 QualiVO hD allg Verw ${ }^{13} ; \mathbb{S} 4$ Abs. 1 ModQV) ${ }^{14}$. Die modulare Qualifizierung ersetzt in den meisten Fällen den vorherigen Verwendungsaufstieg, bietet aber im Gegensatz dazu eine volle Befähigung für alle Ämter der nächsthöheren Laufbahngruppe (mit Ausnahme der Fortbildungsqualifizierung in Rheinland-Pfalz). Noch nicht abschließend geklärt ist die spätere Anerkennung der modularen Qualifizierung in den Bundesländern, welche eine solche Maßnahme nicht vorsehen. Darüber hinaus erkennt sogar Nordrhein-Westfalen nur bis zu 50 Prozent einer modularen Qualifizierung an, welche unter einem anderen Dienstherrn absolviert wurde ( $\$ 6$ Abs. 1 QualiVO hD allg Verw). Es ist zu vermuten, dass an dieser Stelle Mobilitätshemmnisse entstanden sind.

\subsection{Mobilitätsanreize und -hemmnisse für Polizisten}

Auf der Basis der Ergebnisse von geführten Expertengesprächen und Veröffentlichungen der Polizeigewerkschaften wurden Thesen zu Mobilitätsanreizen und -hemmnissen formuliert. Die Gewerkschaften bezogen sich thematisch kurz vor und wenige Jahre nach der Föderalismusreform vor allem auf das neue Besoldungsrecht (vgl. DGB Bundesvorstand 2006b, S. 9; GdP NRW 2010), was vermuten lässt, dass in diesem Bereich Probleme stärker auftreten als im Laufbahnrecht. Dies erscheint schlüssig, da bereits vor der Föderalismusreform die Regelungen für Polizeibeamte in weiten Teilen Ländersache war, es also bereits vielfältige Erfahrungen mit föderalisierten Strukturen

11 Verordnung über die Laufbahnen der Beamtinnen und Beamten im Land Nordrhein-Westfalen (Laufbahnverordnung - LVO) in der Fassung vom 21. Juni 2016, GV. NRW. Nr. 19, ausgegeben am 30.06.2016, S. 441-488.

12 Gesetz über die Leistungslaufbahn und die Fachlaufbahnen der bayerischen Beamten und Beamtinnen (Leistungslaufbahngesetz - LlbG) in der Fassung vom 05. August 2010, GVBl. Bayern Nr. 18, ausgegeben am 12.08.2010, S. 571-604, zuletzt geändert am 13. Dezember 2016, GVBl. Bayern Nr. 19, ausgegeben am 19.12.2016, S. 354-361.

13 Verordnung über den Aufstieg durch Qualifizierung in die Laufbahn des höheren allgemeinen Verwaltungsdienstes im Lande Nordrhein-Westfalen (Qualifizierungsverordnung QualiVO hD allg. Verw.) in der Fassung vom 4. November 2014, GV. NRW. Nr. 34, ausgegeben am 19.11.2014, S. 729-740.

14 Verordnung zur Durchführung der modularen Qualifizierung (Modulare Qualifizierungsverordnung - ModQV) in der Fassung vom 14. Oktober 2011, GVBl Bayern Nr. 21, ausgegeben am 31. 10.2014, S. 538-541. 
vorlagen. Daher wurde die Vorschriftenanalyse im Polizeibereich durch die folgenden vorläufigen Thesen angeleitet:

- Eine höhere Besoldung kann als Mobilitätsanreiz wirken ${ }^{15}$.

- Die unterschiedliche Ausgestaltung der Laufbahngruppen der Polizei wirken als Mobilitätshemmnis ${ }^{16}$.

- Die nichtrechtlich-normierten Absprachen der IMK wirken mobilitätshemmend.

\subsubsection{Laufbahngruppen der Polizei vor und nach der Föderalismusreform I}

Der einfache Dienst verlor im Polizeivollzugsdienst bereits in den 60er Jahren an Bedeutung, sodass deutschlandweit Einstellungen unmittelbar in den mittleren Dienst erfolgten. Auch der mittlere Dienst nahm etwa in Berlin und Hessen bereits vor der Föderalismusreform I eine untergeordnete Rolle ein und wurde nur noch in Übergangsbestimmungen geregelt (siehe z. B. $\$ 21$ (2) SLVO Berlin ${ }^{17}$ ). Nach der Föderalismusreform haben dann weitere Bundesländer den mittleren Dienst abgeschafft. Bremen und Niedersachsen stellen nun direkt in die Laufbahngruppe 2 ein, Rheinland-Pfalz im dritten Eingangsamt, also den vormalig gehobenen Dienst. Nordrhein-Westfalen, Hessen und das Saarland stellen ebenso nicht mehr in den mittleren Dienst ein (vgl. Burmester 2015, S. 20; S. 50).

$\mathrm{Zu}$ Schwierigkeiten bei einem Bundesländer übergreifenden Dienstherrenwechsel kann es insbesondere dann kommen, wenn von einem Bundesland mit mittlerem Dienst in ein Bundesland ohne mittleren Dienst gewechselt werden soll und umgekehrt. Bei einem Wechsel eines Beamten aus dem mittleren Dienst ist demnach eine Beförderung oder ein Aufstieg notwendig, welcher im Zweifel mit Weiterqualifizierungsmaßnahmen und somit einem zusätzlichen Zeitaufwand und/oder Verdienstausfall verbunden sein kann (vgl. z. B. $\$ \$ 23$ ff LVOPolNRW ${ }^{18}$ ). Wechselt ein Beamter aus dem gehobenen Dienst und einem Bundesland ohne mittleren Dienst in ein Bundesland mit mittlerem Dienst, kann auch das zu Qualifizierungsproble-

15 Siehe These Nr. 1.

16 Siehe These Nr. 4; in Kapitel 4.5 formuliert im Hinblick auf alle vier Bereiche.

17 Verordnung über die Laufbahn der Beamtinnen und Beamten der Schutzpolizei (Schutzpolizei-Laufbahnverordnung -SLVO-) in der Fassung vom 12. Juli 1995, GVBl Berlin Nr. 40, herausgegeben am 20.07.1995, S. 453 - 369, zuletzt geändert am 18. Dezember 2012, GVBl Berlin Nr. 32, ausgegeben am 29.12.2012, S. 532-537.

18 Verordnung über die Laufbahn der Polizeivollzugsbeamtinnen und Polizeivollzugsbeamten des Landes Nordrhein-Westfalen (Laufbahnverordnung der Polizei - LVOPol) in der Fassung vom 04. Januar 1995, GV. NRW. Nr. 7, ausgegeben am 31.01.1995, S. 42-47. 
men führen. In Ländern mit mittlerem Dienst ist der gehobene Dienst üblicherweise mit Führungsverantwortung und -fähigkeiten verbunden. Diese können jedoch in einem Bundesland ohne mittleren Dienst nicht flächendeckend alle Beamten des gehobenen Dienstes innehaben.

Eine Eingruppierung bei Einstellung in den gehobenen Dienst kann auf der anderen Seite für viele Polizeianwärter auch als Mobilitätsanreiz wirken, da der gehobene Dienst besser besoldet wird. Gewerkschaften kritisieren jedoch, dass die Höhergruppierung nicht flächendeckend umgesetzt werden kann, weil es an Planstellen mangele. „Beispielhaft sei hier Niedersachsen angeführt, welches 1992 den mittleren Dienst in der Polizeilaufbahn abgeschafft hat und siebzehn Jahre später noch immer ca. 80 Prozent der Beamten sich unterhalb der Planstellenzuweisung nach A 11 befanden“" (Burmester 2015, S. 50; vgl. GdP Niedersachsen 2009, S. 4).

\subsubsection{Nichtrechtlich-normierte Absprachen der IMK}

Grundlegend entscheidend für einen Dienstherrenwechsel im Polizeibereich ist eine Absprache der Innenministerkonferenz, welche zwar nichtrechtlich normiert ist, die jedoch faktisch große Wirkung entfaltet. Der Absprache zufolge darf ein Dienstherrenwechsel über Bundeslandgrenzen hinweg nur im Tauschverfahren stattfinden. Es muss also ein qualifikationsgleicher Polizeibeamter aus einem anderen Bundesland für einen Tausch zur Verfügung stehen. Dieses Tauschverfahren kann sich - wie die Erfahrung lehrt - über viele Jahre ziehen (vgl. GdP MV 2009, S. 8) und birgt die Gefahr, dass der Beamte, welcher seinen Wechselwunsch geäußert hat, von Weiterbildungsmöglichkeiten und Beförderungen ausgeschlossen wird, da der abgebende Dienstherr eine Investition in diesen Beamten für nicht mehr sinnvoll erachtet.

Ein Dienstherrenwechsel ohne Tauschpartner gilt allgemein unter den Ländern als „feindliche Übernahme“ und wird deshalb vermieden ${ }^{19}$. Dem wechselwilligen Polizeibeamten steht es jedoch frei, sich entlassen zu lassen. In diesem Fall sieht jedoch eine Absprache vor, dass der Beamte für ein Jahr nicht in den Polizeidienst eines anderen Dienstherrn eintreten darf, was nicht nur finanzielle, sondern auch statusrechtliche Unsicherheit mit sich bringt.

19 Für einen Praxisfall der feindlichen Übernahme siehe: Bund Deutscher Kriminalbeamter Hamburg (2008): Berlin macht unmoralisches Angebot - In Hamburg sagt man tschüss (https://www.bdk.de/lv/hamburg/aktuelles/berlin-macht-unmoralisches-angebot-in-hambu rg-sagt-man-tsch, Download vom 15.02.2017). 


\subsection{Mobilitätsanreize und -hemmnisse für Lehrer}

Im Lehramtsbereich ergeben sich vor allem Bundesländer übergreifende Unterschiede während des Studiums und des Vorbereitungsdienstes. Diese Unterschiede bestanden schon vor der Föderalismusreform I, da wie im Polizeibereich die Bildung auch zuvor schon Ländersache war. Tabelle 3 gibt einen Überblick über die studierbaren Lehramtstypen ${ }^{20}$ mit den jeweiligen Regelstudienzeiten nach Bundesländern.

Neben den Lehramtstypen unterscheiden sich auch die Anforderungen an die benötigte Fächeranzahl im Studium. Dabei ergeben sich für den Lehramtstyp 1 zum Teil Unterschiede von zwei zu studierenden Fächern in Bayern und vier bis sechs Fächern (je nach Kombination von Haupt- und Nebenfächern) in Hessen. Zu einem Mobilitätshemmnis wird diese heterogene Ausgestaltung vor allem dann, wenn ein Lehramtsanwärter oder bereits angestellter oder verbeamteter Lehrer mit wenigen studierten Fächern in ein Bundesland mit höheren Anforderungen wechseln will.

Ebenfalls problematisch gestaltet sich das Verhältnis von Verbeamtung und einer bloßen Anstellung als Lehrer unter den Bundesländern sowie die unterschiedliche Zuordnung zu Besoldungsgruppen. Als besonders reizvoll werden die Bundesländer eingestuft, welche Lehrer verbeamten. Berlin und Thüringen etwa verbeamten keine Berufsanfänger mehr und Sachsen nur noch mit Einschränkungen.

Auch der Vorbereitungsdienst unterscheidet sich vor allem in den Anforderungen an die Dauer und variiert zwischen 12 und 24 Monaten. Um eine Bundesländerüber greifende Anerkennung von Studium und Vorbereitungsdienst zu gewährleisten, hat die Kultusministerkonferenz (KMK) im Jahr 2013 einen Beschluss verfasst. Demnach soll nicht nur das Studium und die erbrachten Prüfungsleistungen in jedem Bundesland anerkannt werden, sondern auch der geleistete Vorbereitungsdienst, unabhängig von der geforderten Mindestdauer (vgl. KMK 2013, S. 3). Allerdings ist das Fort-

20 „Lehramtstyp 1: Lehrämter der Grundschule bzw. Primarstufe

Lehramtstyp 2: Übergreifende Lehrämter der Primarstufe und aller oder einzelner Schularten der Sekundarstufe I

Lehramtstyp 3: Lehrämter für alle oder einzelne Schularten der Sekundarstufe I

Lehramtstyp 4: Lehrämter der Sekundarstufe II (allgemeinbildende Fächer) oder für das Gymnasium

Lehramtstyp 5: Lehrämter der Sekundarstufe II (berufliche Fächer) oder für das Gymnasium

Lehramtstyp 6: Sonderpädagogische Lehrämter“ (Walm/Wittek 2014, S. 18 ff). 
schreiten des Angleichungsprozesses noch nicht in hinreichendem Maße erkennbar (vgl. Walm/Wittek 2014).

Tabelle 3: Studierbare Lebrämter

\begin{tabular}{|c|c|c|c|c|c|c|c|c|c|c|c|c|c|c|c|c|}
\hline & BW & BY & $\mathrm{BE}$ & BB & HB & $\mathrm{HH}$ & $\mathrm{HE}$ & MV & $\mathrm{NI}$ & NW & $\mathrm{RP}$ & SL & SN & ST & $\mathrm{SH}$ & $\mathrm{TH}$ \\
\hline Lehramtstyp 1 & $\mathrm{x}$ & $\mathrm{x}$ & $\mathrm{x}$ & $\mathrm{x}$ & $\mathrm{x}$ & & $\mathrm{x}$ & $\mathrm{x}$ & & $\mathrm{x}$ & $\mathrm{x}$ & & $\mathrm{x}$ & $\mathrm{x}$ & $\mathrm{x}$ & $\mathrm{x}$ \\
\hline Lehramtstyp 2 & & & & & & $\mathrm{x}$ & & & $\mathrm{x}$ & & & $\mathrm{x}$ & & & & \\
\hline Lehramtstyp 3 & $\mathrm{x}$ & $\mathrm{x}$ & $\mathrm{x}$ & $\mathrm{x}$ & & & $\mathrm{x}$ & $\mathrm{x}$ & $\mathrm{x}$ & $\mathrm{x}$ & $\mathrm{x}$ & $\mathrm{x}$ & $\mathrm{x}$ & $\mathrm{x}$ & $\mathrm{x}$ & $\mathrm{x}$ \\
\hline Lehramtstyp 4 & $\mathrm{x}$ & $\mathrm{x}$ & $\mathrm{x}$ & $\mathrm{x}$ & $\mathrm{x}$ & $\mathrm{x}$ & $\mathrm{x}$ & $\mathrm{x}$ & $\mathrm{x}$ & $\mathrm{x}$ & $\mathrm{x}$ & $\mathrm{x}$ & $\mathrm{x}$ & $\mathrm{x}$ & $\mathrm{x}$ & $\mathrm{x}$ \\
\hline Lehramtstyp 5 & $\mathrm{x}$ & $\mathrm{x}$ & $\mathrm{x}$ & & $\mathrm{x}$ & $\mathrm{x}$ & $\mathrm{x}$ & $\mathrm{x}$ & $\mathrm{x}$ & $\mathrm{x}$ & $\mathrm{x}$ & $\mathrm{x}$ & $\mathrm{x}$ & $\mathrm{x}$ & $\mathrm{x}$ & $\mathrm{x}$ \\
\hline Lehramtstyp 6 & $\mathrm{x}$ & $\mathrm{x}$ & & & $\mathrm{x}$ & $\mathrm{x}$ & $\mathrm{x}$ & $\mathrm{x}$ & $\mathrm{x}$ & $\mathrm{x}$ & $\mathrm{x}$ & & $\mathrm{x}$ & $\mathrm{x}$ & $\mathrm{x}$ & $\mathrm{x}$ \\
\hline
\end{tabular}

Quelle: Walm/Wittek 2014, S. 18; Darstellung von Burmester 2015, S. 28.

Es ist im Lehramtsbereich keine Verschlechterung der Mobilität durch die Föderalismusreform I erkennbar, allerdings auch keine weitreichende Verbesserung zu beobachten. Die unterschiedlichen studierbaren Lehramtstypen, die unterschiedliche benötigte Fächeranzahl, der heterogen ausgestaltete Vorbereitungsdienst und die unterschiedliche Anerkennung von Studium und Vorbereitungsdienst sind nach wie vor Mobilitätshemmnisse und -hürden.

\subsection{Thesen und Hypothesen}

Mangels einer bereits ausgearbeiteten Theorie bzw. allenfalls punktuell argumentierender Literatur wurde vorstehend das aktuelle Dienstrecht analysiert, um Hinweise auf mögliche Mobilitätsanreize und Mobilitätshindernisse zu gewinnen. Mögliche Mobilitätsanreize ergeben sich tatsächlich bereits aus der Literatur (siehe Kapitel 1) und der Analyse der unterschiedlich hohen Besoldung in den Bundesländern (siehe Kapitel 3). Auf dieser Basis lässt sich die These Nr. 1 formulieren, dass die unterschiedlichen Besoldungshöhen einen merklichen Anreiz bilden, in diejenigen Bundesländer zu wechseln, die vergleichsweise gut besolden. Wie aus den in der Einleitung formulierten ersten grundsätzlichen Überlegungen hervorgeht, kann auch von einem attraktiven Dienstrecht, das attraktive Weiterbildungs- und Aufstiegsmöglichkeiten bietet, ein Anreiz für einen Wechsel des Bundeslandes ausgehen. Der entsprechende Zusammenhang soll hier in These Nr. 2 abgebildet werden, nachdem von einem attraktiven Dienstrecht merkliche Anreize für einen Bundeslandwechsel ausgehen. 
Umgekehrt sollte mit der vorstehenden Vorschriftenanalyse ${ }^{21}$ deutlich geworden sein, dass es in den Bereichen Polizei, Lehramt und allgemeine Verwaltung infolge der entstandenen Hürden zu Schwierigkeiten bei einem Bundesländer übergreifenden Dienstherrenwechsel kommen kann. Problematisch scheint insbesondere die Fragmentierung der Aufstiegs- und Beförderungsregelungen zu sein (siehe Kapitel 4.2). Ähnliches gilt für das Ausmaß der Anerkennung von durchlaufener Weiterbildung (siehe Kapitel 4.2). Auch wenn hier die reine Vorschriftenanalyse keine hinreichenden Hinweise auf die tatsächliche Praxis geben kann, darf jedoch vermutet werden, dass es zu Problemen bei der Anerkennung von in einem anderen Bundesland erworbenen Qualifikationen bei einem Bundeslandwechsel kommen kann. Zieht man beide angesprochenen Bereiche zusammen und bezieht sich auf alle sich zunehmend fragmentiert darstellenden Vorschriften, kann die These Nr. 3 formuliert werden, dass die unterschiedlichen Vorschriften der Bundesländer einen Bundesländer übergreifenden Dienstherrnwechsel verkomplizieren.

Insbesondere Beamten der allgemeinen Verwaltung scheint eine Verschlechterung der Mobilitätsmöglichkeiten durch die zunehmende Ausdifferenzierung des Laufbahnrechts zu drohen. Bei genauerer Analyse der verschiedenen Laufbahnsysteme wurde deutlich, dass sich die Zahl der Laufbahngruppen im Bundesländervergleich sehr unterschiedlich darstellt. Das System ist auf den ersten Blick also fragmentiert. Bei genauerer Betrachtung weisen alle Systeme aber nach wie vor vier unterschiedliche Eingangsämter auf. Der Zugang zu ihnen ist nach wie vor an bestimmte Qualifikationen geknüpft ist. Folglich dürfte - so die hier formulierte These Nr. 4 - die unterschiedliche Zahl an Laufbahngruppen im Vergleich der Bundesländer keine nennenswerten Hemmnisse bei einem Bundesländergrenzen überschreitenden Wechsel mit sich bringen (siehe Kapitel 4.1).

Im Lehramtsbereich scheint das Hauptproblem in der heterogenen Ausgestaltung von Studium und Vorbereitungsdienst zu liegen. Hieraus lässt sich These Nr. 5 ableiten, dass die Nichtanerkennung der erworbenen Ausbildung zu einem Mobilitätshemmnis werden kann.

Im Polizeibereich können Probleme vor allem durch das Tauschverfahren und die informelle Absprache der IMK erwartet werden. Da auch im Lehramtsbereich Tauschverfahren zum Einsatz kommen und auch für den Bereich der allgemeinen Verwaltung zumindest diskutiert, wenn nicht bereits praktiziert werden, soll hier die These Nr. 6 formuliert werden, dass das Tauschverfahren einen Wechsel des Bundeslandes aufwendig macht.

21 Hierbei handelt es sich um eine knappe Zusammenfassung der von Burmester vorgelegten, ausführlichen Fassung. 
Wegen des gewählten vergleichenden Ansatzes, der durch den Vergleich der zwei bereits seit geraumer Zeit föderalisierten Bereiche der Polizei und des Lehramts einerseits mit den weniger lang föderalisierten Bereichen der allgemeinen Verwaltung und der Finanzverwaltung charakterisiert ist, lassen sich auch Fragen beantworten, die darauf zielen, Unterschiede zwischen den beiden Gruppen offenzulegen. Generell wurde bei der Anlage der Studie davon ausgegangen, dass die bereits länger föderalisierten Bereiche in intensiverem Maße mit den Problemen zunehmender Fragmentierung konfrontiert sind. Diese Bereiche sind also gewissermaßen ein Abbild der zukünftigen Entwicklung in den neu föderalisierten Bereichen. Dagegen lässt sich argumentieren, dass die Verwaltung in diesen bereits länger föderalisierten Bereichen mehr Erfahrung gesammelt hat und folglich besser mit der Situation umgehen kann als dies in den neu föderalisierten Bereichen der Fall ist; dort müssen viele Erfahrungen erst gesammelt werden. Da wir davon ausgehen, dass die Probleme mit zunehmender Fragmentierung zunehmen, neigen wir insgesamt der These vom Abbild zukünftiger Entwicklungen zu. Allerdings dürfte eine Zunahme der Probleme im Zeitverlauf ein umgekehrtes Bild zeigen. Denn die bislang wenig oder nicht föderalisierten Bereiche dürften im Zeitverlauf ausgeprägter mit einer Zunahme an Problemen konfrontiert sein als die schon länger föderalisierten Bereiche, für die sich folglich nur wenig geändert hat. Hieraus ergeben sich die folgenden Hypothesen:

- Hypothese A: Von den Beamten in den schon seit längerem föderalisierten Bereichen Polizei und Lehramt wird ein Versetzungsverfahren als komplizierter eingeschätzt als von den Beamten der erst kürzlich föderalisierten Bereiche allgemeine Verwaltung und Finanzverwaltung.

- Hypothese B: Von den Beamten in den schon seit längerem föderalisierten Bereichen Polizei und Lehramt wird ein Tauschverfahren als aufwendiger eingeschätzt als von den Beamten der erst kürzlich föderalisierten Bereiche allgemeine Verwaltung und Finanzverwaltung.

- Hypothese C: Bei den bislang wenig föderalisierten Bereichen der allgemeinen Verwaltung und der Finanzverwaltung ist mit einer stärkeren Zunahme der Probleme bei einem Bundesländergrenzen übergreifenden Dienstherrenwechsel zu rechnen als bei den bereits länger föderalisierten Bereichen Polizei und Lehramt. 
Die formulierten Thesen und Hypothesen werden im Rahmen der nachfolgend dokumentierten Online-Befragung überprüft. Unabhängig von einer solchen Befragung lässt sich bereits jetzt festhalten, dass wegen des höheren Fragmentierungsgrades der Vorschriften erhöhte Informationskosten bei einem geplanten Dienstherrenwechsel auf die Beamten zukommen. 


\section{Online-Umfrage}

Die Online-Umfrage als Befragungsmethode gilt allgemein als anwenderfreundlich, da mit vergleichsweise wenig Zeitaufwand eine große Anzahl von Menschen erreicht werden kann. Die vorzuschaltenden Grundüberlegungen und die methodischen Anforderungen sind jedoch denen anderer Befragungsmethoden ähnlich (vgl. Pötschke 2009, S. 81). Sobald der Forschungsgegenstand bestimmt und die Forschungsfragen, die Thesen oder Hypothesen ausformuliert worden sind, muss zunächst die zu untersuchende Grundgesamtheit definiert werden. Während bei den meisten Befragungen aus arbeitsökonomischen Gründen mit einer Stichprobenziehung gearbeitet wird, wurde für die im Folgenden dokumentierte Umfrage zur Mobilität von Beamten der allgemeinen Verwaltung und Finanzverwaltung, von Lehrern und Polizisten eine Vollerhebung aller bekannten Experten in der öffentlichen Verwaltung selbst angestrebt (siehe Kapitel 2.1). Als Grundgesamtheit wurden alle personalverantwortlichen Stellen der obersten Landesbehörden und der nachgeordneten staatlichen Verwaltung definiert ${ }^{22}$.

Im nachfolgenden Kapitel 5.1 wird auf die Mobilitätsanreize eingegangen und in Kapitel 5.2 auf die möglichen Hemmnisse und Hürden bei einem Dienstherrenwechsel über Bundesländergrenzen hinweg. Die Anerkennung von Ausbildung und Fortbildungen im aufnehmenden Bundesland ist Gegenstand der Darstellungen in Kapitel 5.3; es schließt sich eine Einschätzung der Entwicklungstendenzen der vergangenen Jahre an. Kapitel 5.4 ist Wechselproblemen im Zeitverlauf gewidmet.

22 Es sind im Rahmen der Online-Umfrage bewusst nicht die Beamten selbst befragt worden, da diese größtenteils nicht über die spezifischen Verfahrensabläufe informiert sind, die hier u. a. von Interesse sind. Des Weiteren wäre eine klare Festlegung der Grundgesamtheit (alle Lehrer, Polizisten und Beamte der allgemeinen Verwaltung und der Finanzverwaltung oder alle, die schon einmal den Wunsch hatten zu wechseln oder alle, die schon einmal gewechselt sind) nicht möglich. Auch hätten die Beamten im Rahmen des gegebenen Datenschutzes kaum angesprochen werden können. In Verbindung mit den durchgeführten Fallstudien, mit denen die Perspektive der unmittelbar Betroffenen berücksichtigt wird, ist jedoch eine multiperspektivische Auswertung der Mobilitätssituation möglich. 


\subsection{Mobilitätsmotive}

Abbildung 5: Gründe für einen Bundeslandwechsel (Mehrfachnennungen möglich)

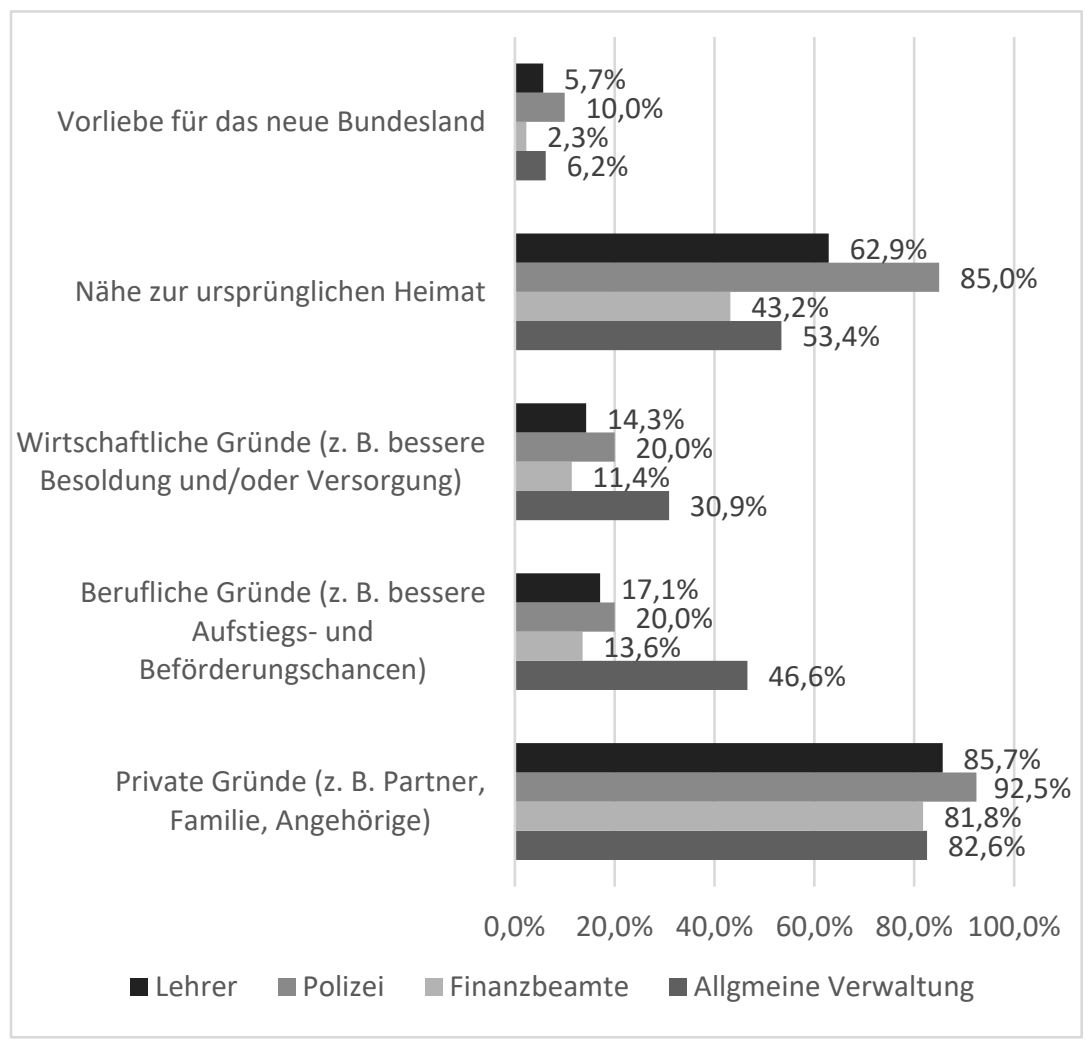

Quelle: Eigene Datenerhebung und eigene Berechnung.

Föderale Strukturen bergen die Chance oder das Risiko eines Wettbewerbs der Bundesländer untereinander (Dose 2016, S. 28). Unter den Bedingungen des derzeit schrumpfenden Angebots an Arbeitskräften (siehe Kapitel 1) bedeutet dies auch einen verschärften "Wettbewerb um die besten Köpfe“ (Schäuble im Interview: DGB Bundesvorstand 2006a, S. 1) nicht nur mit den Arbeitgebern der Privatwirtschaft, sondern auch zwischen Bund, Län- 
dern und Kommunen. Wie dargelegt kann von unterschiedlichen Besoldungshöhen ein substantieller Anreiz für einen Wechsel des Bundeslandes ausgehen (siehe These 1). Auch ein attraktives Laufbahnsystem könnte ein Vorteil in diesem Wettbewerb sein. Von ihm könnte gleichfalls ein Mobilitätsanreiz für einen Wechsel in ein anderes Bundesland ausgehen (siehe These 2). Zur Überprüfung der Thesen 1 und 2 wurde in der Umfrage nach Mobilitätsanreizen für Beamte gefragt. Neben den vorgegebenen Antwortmöglichkeiten, wie sie in Abbildung 5 dokumentiert sind, konnten auch weitere Gründe im Rahmen offener Antwortmöglichkeiten benannt werden. Diese Möglichkeit wurde allerdings von lediglich zwei Befragungsteilnehmern genutzt, die damit die bereits vorher gewählte Antwort nochmals unterstreichen wollten.

In Abbildung 5 sind die angegebenen Gründe für einen Bundeslandwechsel nach Beschäftigungsbereichen der Beamten aufgeschlüsselt dargestellt. Bei der Beantwortung der Frage waren Mehrfachantworten möglich. Die privaten Gründe sind demnach ein Hauptanreiz, um einen Bundesländer übergreifenden Dienstherrenwechsel anzustreben. Die Nähe zur ursprünglichen Heimat erreicht die zweithöchsten Prozentwerte, vor allem bei Polizeibeamten. Die Thesen 1 und 2 können damit nicht verworfen werden. Von ihnen gehen jedoch nicht die stärksten Anreize für einen Wechsel des Bundeslandes aus. Für die allgemeine Verwaltung sehen jedoch immerhin 30,9 Prozent der Personalverantwortlichen in der allgemeinen Verwaltung wirtschaftliche Gründe als Grund für einen Bundeslandwechsel. Weitere berufliche Gründe wie bspw. bessere Aufstiegsmöglichkeiten werden sogar von 46,6 Prozent der Befragten als Wechselgrund gesehen.

Das grundsätzliche Bild deckt sich mit den Ergebnissen aus den Fallstudien (siehe Kapitel 6), wonach sich Polizeianwärter nicht selten für eine Ausbildung in mehreren Bundesländern bewerben und nach abgeschlossenem Vorbereitungsdienst in einem anderen als dem Heimatbundesland versuchen, im Heimatbundesland einen Tauschpartner für einen Wechsel zu finden.

Im weiteren Verlauf der Befragung wurden die Teilnehmenden gebeten, sich auf einen Hauptwechselgrund festzulegen. Wie die Werte in Abbildung 6 zeigen, fällt ein Hauptteil der Stimmen auf die privaten Gründe (insgesamt 77,2 Prozent). Dabei liegen alle Beamtenbereiche bei über 87 Prozent; einzige Ausnahme bildet die allgemeine Verwaltung mit nur 70,4 Prozent. Die Nähe zur ursprünglichen Heimat erreicht bei den Polizisten über 10 Prozent. Interessant ist der Wert von knapp 12 Prozent, der bei der allgemeinen Verwaltung für die beruflichen Gründe erreicht wird. Ganz offensichtlich werden die beruflichen Bedingungen im Bundesländervergleich als so unterschiedlich wahrgenommen, dass dies ein Hauptgrund für einen Wech- 
sel sein kann. Werden die Antwortmöglichkeiten „berufliche Gründe“ und „wirtschaftliche Gründe“ zusammengefasst, ergeben sich die Werte, wie sie in Abbildung 7 dargestellt werden.

\section{Abbildung 6: Hauptgrund für einen Bundeslandwechsel (Ohne Mehrfachnennungen)}

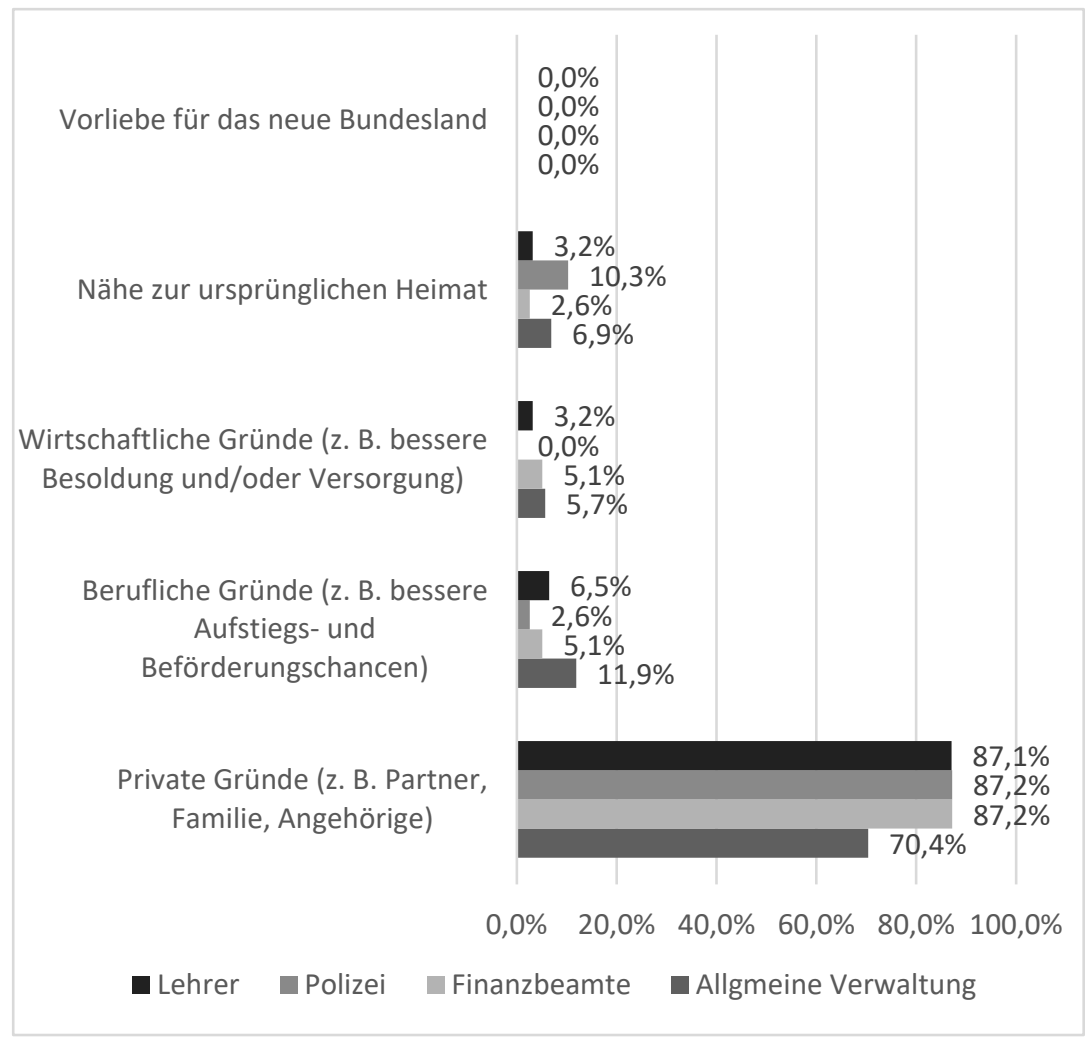

Quelle: Eigene Datenerhebung und eigene Berechnung. 
Abbildung 7: Berufliche und wirtschaftliche Gründe als Hauptmobilitätsanreiz

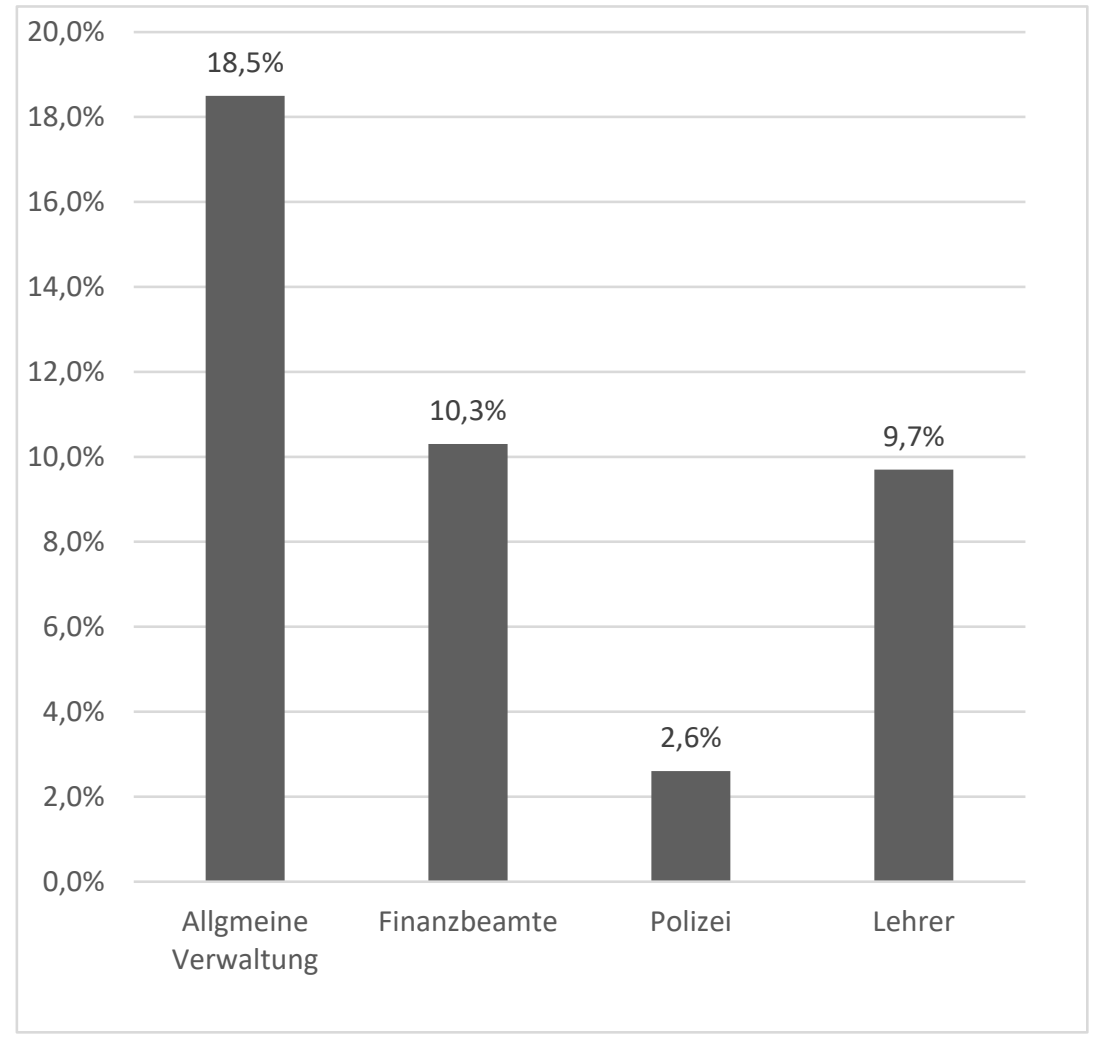

Quelle: Eigene Datenerhebung und eigene Berechnung.

Demnach waren bessere Aufstiegs- und Beförderungschancen oder eine bessere Besoldung und Versorgung vor allem für Beamte der allgemeinen Verwaltung Anlass, die Versetzung in ein anderes Bundesland anzustreben. Insbesondere Polizeibeamte wechseln hingegen kaum, um die entsprechenden wirtschaftlichen Vorteile wahrzunehmen. Ähnliches gilt weniger ausgeprägt für Lehrer und Finanzbeamte. Offen bleibt dabei allerdings, ob die Ergebnisse so zustande kamen, weil kaum wirtschaftliche Vorteile gesehen wurden oder ob diese durchaus bejaht werden, sie aber kaum Einfluss auf die jeweilige persönliche Entscheidung haben. Trotz dieser Unklarheit kann ge- 
schlussfolgert werden, dass vor allem im Bereich der allgemeinen Verwaltung für die Gesetzgeber die Chance besteht, mit einem attraktiven Laufbahnsystem und einer hohen Besoldung wirksam in den Wettbewerb um Personal einzutreten und sich Vorteile gegenüber anderen Bundesländern zu verschaffen.

\subsection{Mobilitätshemmnisse und -hürden}

Wenn in einem gewissen Umfang Anreize bestehen, das Bundesland zu wechseln, heißt dies noch nicht, dass es auch tatsächlich zu einem solchen Wechsel kommt. Es gilt die Faktoren zu beachten, die sich als Hürden erweisen können. Sie müssen bei bestehenden Anreizen erst überwunden werden, bevor ein Wechsel realisiert werden kann. An den verschiedenen Faktoren, die eine solche Hürde konstituieren können, waren wir insbesondere interessiert, wobei uns auch an der relativen Bedeutung dieser Einflüsse gelegen war. Grundsätzlich können Hürden und Probleme auf institutioneller oder individueller Ebene entstehen. Zu den institutionellen Faktoren zählen ein undurchsichtiges, kompliziertes oder auch ungerechtes Verfahren sowie auch dem Wechsel entgegenstehende materielle Vorschriften. Zu den individuellen Faktoren zählen zum einen Eigenschaften des Beamten wie der gesundheitliche Zustand oder die Persönlichkeit, aber auch subjektive Wahrnehmungen sowohl der wechselwilligen Beamten als auch der Dienstherren.

\subsubsection{Mögliche Ablehnungsgründe auf Seiten der aufnehmenden Dienstbehörden}

Diejenigen Faktoren, die eine Dienstbehörde veranlassen, sich gegen einen Versetzungsantrag zu entscheiden, sollen nachfolgend dargestellt und analysiert werden. In Abbildung 8 werden - differenziert nach Beamtengruppen - die Häufigkeiten der Nennungen im Bereich der institutionellen Gründe präsentiert und in Abbildung 9 werden die Häufigkeiten für die individuellen Gründe wiedergegeben. Im Fragebogen wurden für die Teilnehmer individuelle und institutionelle Gründe nicht explizit unterschieden; es wurde lediglich nach den einzelnen Items gefragt. Die Befragten hatten dabei die Möglichkeit, Mehrfachantworten zu geben. Wichtig für eine Bewertung der Ergebnisse ist, dass es sich nicht um eine Auszählung der tatsächlichen Ablehnungsgründe aus der Vollzugspraxis handelt, sondern um eine Einschätzung der Befragten über die Gründe für einen Wechsel und deren relative Bedeutung. 
Abbildung 8: Institutionelle Gründe für die Ablehnung von Versetzungsanträgen seitens der Dienstbehörde (Mehrfachnennungen möglich)

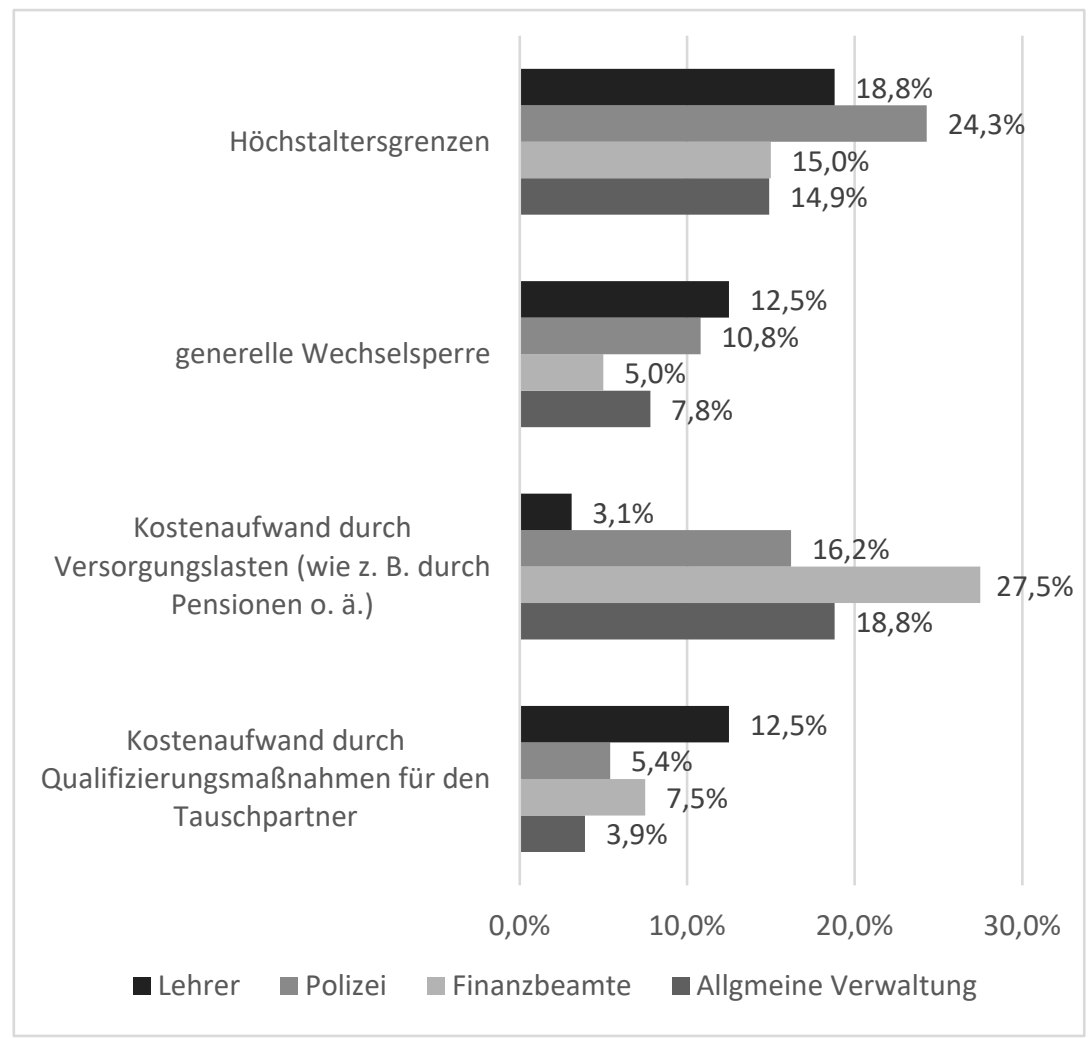

Quelle: Eigene Datenerhebung und eigene Berechnung.

$\mathrm{Zu}$ den institutionellen Gründen, welche als Antwortmöglichkeiten im Fragebogen vorgegeben waren, gehörten der Kostenaufwand durch Weiterqualifizierungsmaßnahmen für den Tauschpartner aus einem anderen Bundesland, Kostenaufwand durch Versorgungslasten, Höchstaltersgrenzen sowie schließlich eine generelle Wechselsperre. Auch wenn das Alter eines Beamten zu den individuellen Eigenschaften zählt, ist die Höchstaltersgrenze für eine Verbeamtung keine natürliche Grenze, sondern bundesweit in den Laufbahnverordnungen der Länder unterschiedlich ausgestaltet. Die Vor- 
schriften in diesem Bereich sind also bereits fragmentiert und wirken deshalb in den verschiedenen Bundesländern unterschiedlich. Darüber hinaus gelten für Polizisten in den meisten Bundesländern Höchstaltersgrenzen für die Einstellung in den Vorbereitungsdienst und Höchstaltersgrenzen für Aufstiege (vgl. Burmester 2015, S. 23, 47, 51). Das führt zu der Vermutung, dass vor allem im Polizeibereich Höchstaltersgrenzen verstärkt als Hürden bei Bundesländergrenzen überschreitendem Dienstherrnwechsel wirken können.

Wie Abbildung 8 zeigt, weist die Höchstaltersgrenze bei Polizisten im Vergleich mit den anderen Beamtengruppen tatsächlich die häufigsten Nennungen auf. Demnach sind 24,3 Prozent der Befragten, welche für Polizeibeamte zuständig sind, der Meinung, dass Höchstaltersgrenzen durchaus bedeutsamer Grund für die Ablehnung eines Versetzungsantrags sind. Die Kosten von Weiterqualifizierungsmaßnahmen für den Tauschpartner ist nach Einschätzung der Befragten hingegen nicht häufiger Grund einer Ablehnung von Versetzungsanträgen. Der Kostenaufwand durch Versorgungslasten wird besonders bei Finanzbeamten häufig als Ablehnungsgrund genannt, während dieser Aspekt bei Lehrern eine eher geringe Rolle zu spielen scheint. Die generelle Wechselsperre hat Beamtengruppen übergreifend gleichfalls eine geringe Bedeutung. Unter allen Befragten gaben nur 8,7 Prozent an, dass ihr Dienstherr überhaupt schon einmal eine generelle Wechselsperre verhängt habe.

Insgesamt betrachtet wird den institutionellen Faktoren von den befragten Teilnehmern keine signifikante Bedeutung beigemessen. Abgesehen von dem Kostenaufwand durch Versorgungslasten bei Finanzbeamten und den Höchstaltersgrenzen bei Polizisten wurden alle Faktoren von weniger als 20 Prozent der Befragten als Ablehnungsgrund genannt.

Abbildung 9 zeigt die Verteilung der Antworten zu den in der Person der jeweiligen wechselwilligen Beamten liegenden Gründe für eine Ablehnung des Antrags auf Versetzung in ein anderes Bundesland durch die Dienstbehörde. 62,5 Prozent der zuständigen personalverantwortlichen Befragten für den Bereich Lehramt gehen davon aus, dass die Unabkömmlichkeit des eigenen Beamten ein potentieller Grund der Ablehnung eines Versetzungsantrages sein kann. Ein möglicher Ansatz zur Erklärung dieses Befundes könnte sein, dass nicht nur ein Tauschpartner mit der gleichen Fächerkombination gefunden werden muss, sondern der abzugebende Lehrer unter Umständen noch zusätzliche Aufgaben wie die Betreuung von Arbeitsgemeinschaften oder Sportangeboten innehat. Ebenso kann aber auch der weit verbreitete Mangel an Lehrkräften Grund für die Einschätzung sein, dass ein wechselwilliger Lehrer unabkömmlich sei. Dies dürfte insbesondere für Mangelfächer unterrichtende Lehrer gelten. Auch bei Beamten 
der allgemeinen Verwaltung wird die Unabkömmlichkeit von 48,7 Prozent der Befragten als Ablehnungsgrund genannt.

Abbildung 9: Individuelle Gründe für die Ablehnung von Versetzungsanträgen seitens der Dienstbehörde (Mehrfachnennungen möglich)

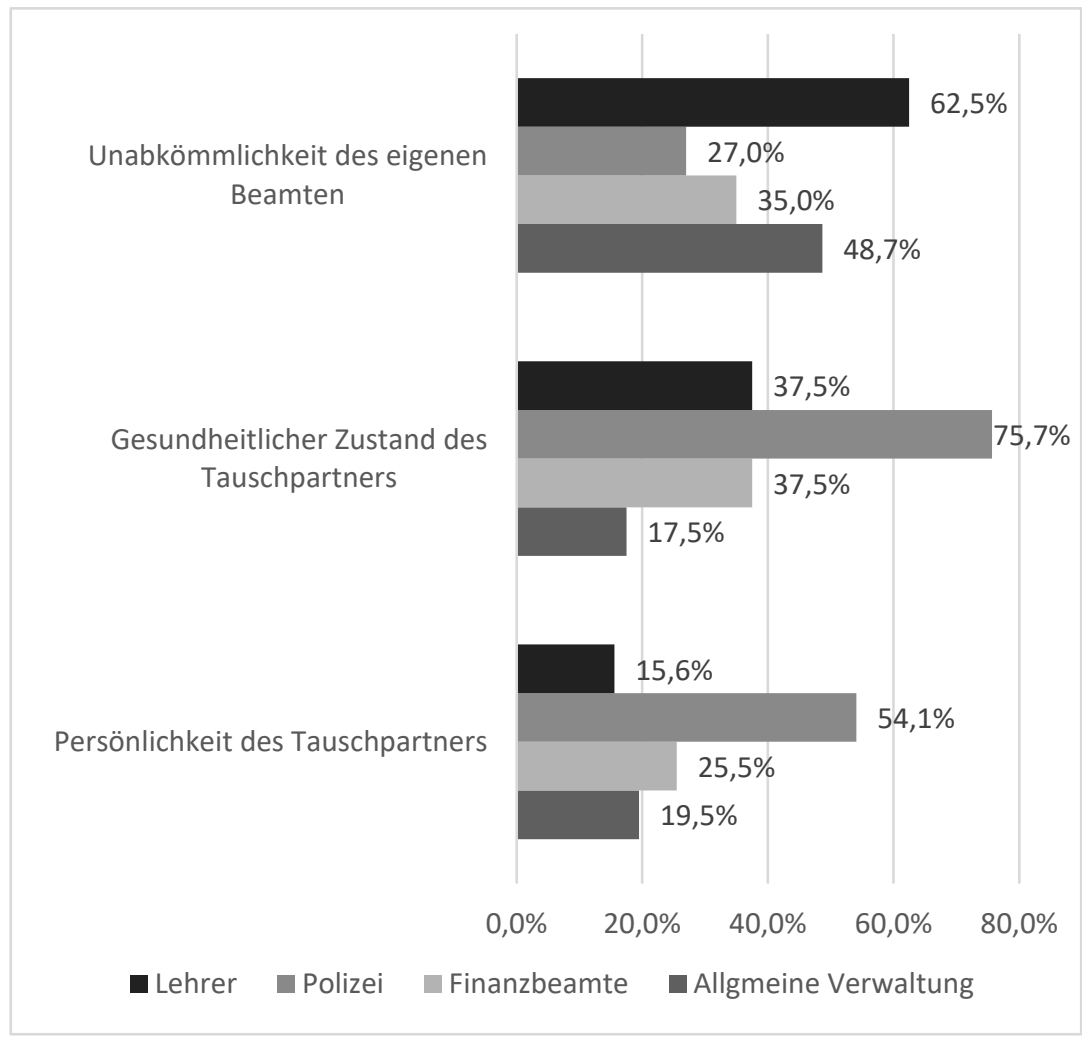

Quelle: Eigene Datenerhebung und eigene Berechnung.

Dass der gesundheitliche Zustand des Tauschpartners von mehr als 75 Prozent der personalverantwortlichen Mitarbeiter im Bereich der Polizei als Ablehnungsgrund ausgewählt wurde, ist wenig überraschend. Vor allem im mittleren und gehobenen Dienst erfordert der Polizeiberuf gegenüber ande- 
ren Beamtengruppen die höchste körperliche Fitness. Diese wird auch bei jedem Wechsel geprüft; in aller Regel wird eine erneute Gesundheitsprüfung gefordert. Die Persönlichkeit des Tauschpartners wird ebenfalls insbesondere im Bereich der Polizei als Ablehnungsgrund gesehen. Während bei den Lehrern lediglich 15,6 Prozent der Befragten hier einen Ablehnungsgrund erkennen, liegt dieser Wert bei der Polizei bei 54,1 Prozent. Dieser vergleichsweise hohe Wert im Bereich der Polizei könnte durch das hohe $\mathrm{Maß}$ an gegenseitigem Vertrauen, das speziell bei Außeneinsätzen der Polizei notwendig ist, begründet sein. Insgesamt erreichen die Unabkömmlichkeit des antragstellenden Beamten im Lehramtsbereich und die Gesundheit und Persönlichkeit des Tauschpartners im Polizeibereich die eindeutig höchsten Werte.

Da sich das Antwortverhalten im Vergleich der Behördenebenen nur marginal unterscheidet, kann davon ausgegangen werden, dass die Wahrnehmung der Ablehnungsgründe in den obersten Landesbehörden sich nicht maßgeblich von der in den nachgeordneten Behörden unterscheidet. In einem Textfeld wurde darüber hinaus die Möglichkeit gegeben, weitere Ablehnungsgründe anzugeben. Diese Möglichkeit wurde vor allem von Teilnehmern der Umfrage aus den obersten Landesbehörden genutzt. Dabei wurde mehrfach auf einen fehlenden Tauschpartner hingewiesen und dies Beamtengruppen übergreifend. Unter anderem wurden abweichende Statusämter der Tauschpartner (Polizei), dienstliche Beurteilung und laufende dienstrechtliche Maßnahmen (Polizei), laufbahnrechtliche Rahmenbedingungen (Polizei), unpassende Qualifikationen des Tauschpartners (allgemeine Verwaltung), fehlende Einstellungsvoraussetzungen (allgemeine Verwaltung), personalwirtschaftliche Gründe (allgemeine Verwaltung), abweichende Qualifizierungswege (Lehrer) und Ungeeignetheit für die bundesweit ausgeschriebene Stelle (Finanzen) genannt.

\subsubsection{Mögliche Hemmnisse auf Seiten der Beamten}

Bevor in der Umfrage einzelne, spezifische Schritte des Versetzungsverfahrens thematisiert wurden, wurde nach einer generellen Einschätzung des Verfahrens bei einer Bundesländer übergreifenden Versetzung gefragt. Auf einer fünfstufigen Skala ${ }^{23}$, die von ziemlich kompliziert (5) bis ziemlich unkompliziert (1) reichte, sollten die Befragten ihre Beurteilung des Verfahrens verorten. Hintergrund der gestellten Frage war die Hypothese A, dass die

23 Die Skala umfasst die Ausprägungen „ziemlich kompliziert“, „eher kompliziert“, „teils/ teils“, „eher unkompliziert“, „ziemlich unkompliziert“ und zusätzlich die Kategorie „weiß nicht“. 
bereits stark föderalisierten Beamtengruppen der Lehrer und Polizisten das Verfahren als komplizierter einschätzen als die neu föderalisierten Beamtengruppen. Hypothese A kann durch die Befragung nicht bestätigt werden. Diese Einschätzung soll im Folgenden näher erläutert werden.

Abbildung 10: Komplexität des Versetzungsverfahrens

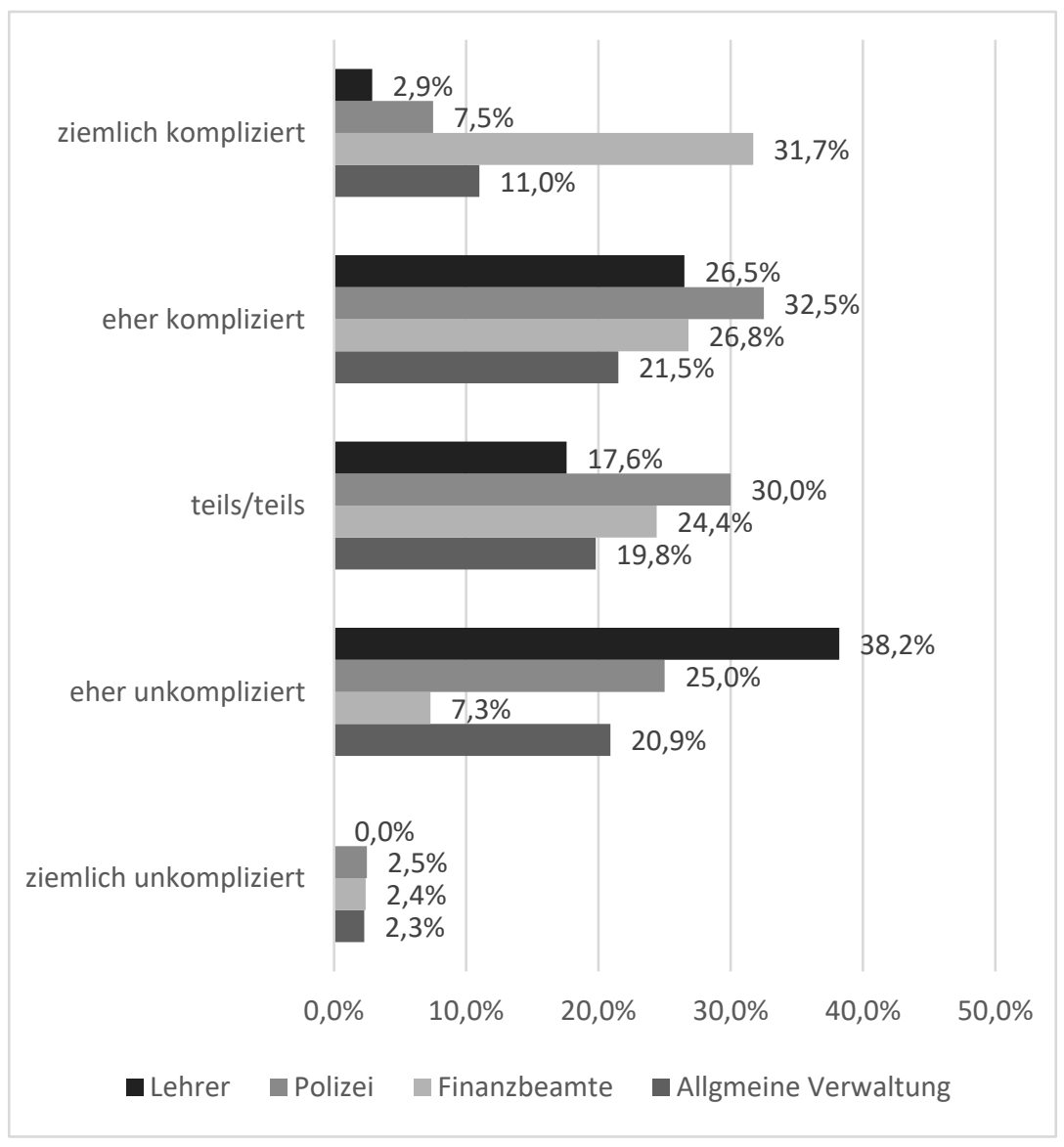

Quelle: Eigene Datenerhebung und eigene Berechnung. 
Abbildung 10 zeigt die Verteilung der Antworten differenziert nach den Beamtengruppen. Für die Finanzbeamten wird das Versetzungsverfahren als besonders kompliziert eingestuft. Hier nannten 31,7 Prozent der Befragten das Verfahren ziemlich kompliziert und nur 7,3 Prozent eher unkompliziert. Für die Gruppe der Lehrer stellt sich das Verfahren hingegen als besonders unkompliziert dar. Hier gaben nur 2,9 Prozent der Befragten an, das Versetzungsverfahren sei ziemlich kompliziert, während hingegen 38,2 Prozent der Befragten mitteilten, dass das Verfahren eher unkompliziert sei. Die Befragten aus der allgemeinen Verwaltung und der Polizei liegen mit ihren Antworten dazwischen, wobei die allgemeine Verwaltung das Verfahren tendenziell als etwas komplizierter einschätzt.

So gaben 20,9 Prozent der Befragten aus der allgemeinen Verwaltung an, dass das Verfahren eher unkompliziert sei, während 11 Prozent antworteten, dass Verfahren sei ziemlich kompliziert. Bei den Befragten der Polizei gaben 25 Prozent an, das Verfahren sei eher unkompliziert und 7,5 Prozent votierten für ziemlich kompliziert. Die Kategorie ,ziemlich unkompliziert wurde Beamtengruppen übergreifend von maximal 2,5 Prozent der Befragten genannt. Somit muss Hypothese A verworfen werden, da tendenziell die bereits stärker föderalisierten Beamtengruppen, wie Lehrer und Polizisten, das Verfahren als unkomplizierter einschätzen.

Auf die Frage, ob einige Versetzungsanträge gar nicht erst gestellt werden, obwohl der Wunsch nach einem Bundeslandwechsel vorhanden ist, antworteten etwa 20 Prozent der Befragten mit „Ja“. Der gegenteiligen Meinung waren 23,5 Prozent der Befragten und alle Übrigen antworteten mit „weiß nicht“. Von allen Befragten, welche diese Frage bejahten, waren bei der Möglichkeit von Mehrfachnennungen etwa

- 70 Prozent der Meinung, dass der Antrag aufgrund von niedrigen erwarteten Erfolgschancen nicht gestellt werde;

- 34 Prozent der Meinung, dass das Verfahren als kompliziert eingeschätzt werde;

- 19 Prozent der Meinung, dass der Beamte einen zu hohen Aufwand vermute und

- 11 Prozent gaben an, dass die Dienstbehörde ausdrücklich von einer Antragstellung abrate.

Die in der Aufzählung genannten Gründe waren den Befragten bereits vorgegeben. Ergänzend konnten noch weitere Gründe als offene Antwort formuliert werden. Eine Auswertung der Antworten auf die entsprechende offene Frage macht deutlich, dass vor allem im Polizeibereich Ängste vor Diskriminierung und dienstlicher Benachteiligung die Beamten von einem Versetzungsantrag abhalten können. Ausdrücklich genannt werden dabei die 
Beförderungschancen auf der aktuellen Dienststelle. Dieses Ergebnis deckt sich erneut mit den Ergebnissen der Fallstudien (siehe Kapitel 6). Aus Perspektive der im Rahmen der Fallstudien interviewten Beamten und der an der Online-Umfrage teilnehmenden, personalverantwortlichen Mitarbeiter scheint eine gewisse Zurückhaltung der Dienstherren zu bestehen, in wechselwillige Beamte und deren Weiterbildung zu investieren.

Die genannten Gründe sind auf der individuellen Ebene der Hemmnisse und Hürden zu verorten, die zudem auf subjektiven Wahrnehmungen von Chancen auf Erfolg im Vorfeld des Verfahrens basieren. Ein institutionelles Problem liegt dann vor, wenn die Erfolgschancen auf einen Wechsel tatsächlich gering ausfallen und das Verfahren zu kompliziert ist. Für die Erfassung wird hier allerdings - wie bei den anderen Fragen auch - auf die Einschätzung von Personalverantwortlichen rekurriert. In vorwegnehmender Andeutung der weiter unten formulierten Handlungsempfehlungen lässt sich hier anfügen, dass individuellen Hemmnissen durch institutionelle Anpassungen entgegengewirkt werden könnte, etwa durch Informationsangebote der Dienstherren. Auch eine offene Personalpolitik, die einen Beamten durch seinen Wechselwunsch nicht in eine benachteiligende Situation bringt, kann die wechselwilligen Beamten unterstützen.

\subsubsection{Tauschpartner}

Das Tauschverfahren für Lehrer ist in einem Beschluss der Kultusministerkonferenz (KMK) aus dem Jahr 2002 geregelt (siehe ausführlicher Kapitel 6.2.2). Ob Finanzbeamte und Beamte der allgemeinen Verwaltung in der Vollzugspraxis einen Tauschpartner für einen Bundeslandwechsel benötigen, war im Vorfeld der Befragung jedoch unklar. Daher wurden die personalverantwortlichen Mitarbeiter für diese Beamtengruppen in der Umfrage explizit gefragt, ob in ihrem Geschäftsbereich ein Tauschpartner notwendig sei. Über alle Behördenebenen und Bundesländer hinweg ließ sich in diesem Punkt keine einheitliche Vollzugspraxis feststellen: Für ein und dieselbe Beamtengruppe im gleichen Bundesland und auf gleicher Behördenebene gaben einige Befragte an, dass ein Tauschpartner für einen Wechsel erforderlich sei, während andere dies verneinten. Die Notwendigkeit scheint demnach von der einzelnen Behörde oder aber dem individuellen Personalbedarf abzuhängen.

Auf die Frage, wie die Suche nach einem Tauschpartner einzuschätzen sei, antworteten 67,9 Prozent aller Befragten mit „ziemlich/eher aufwendig“, wie dies in Tabelle 4 nochmals im Kontext dargestellt ist. Nur 1,8 Prozent der Teilnehmer gaben an, dass das Finden eines Tauschpartners „eher unaufwendig" sei. Die übrigen Antworten verteilten sich auf die Antwortmöglich- 
keit „teils/ teils“ (19,3 Prozent) und „weiß nicht“ (11 Prozent). Die Antwortmöglichkeit „ziemlich unaufwendig“ wurde von keinem der Befragten gewählt.

Es zeigt sich, dass die Befragten aus den Landesmittelbehörden vergleichsweise am wenigsten Aufwand bei der Suche nach Tauschpartnern sahen, aber dennoch mit 50 Prozent im Bereich „ziemlich/eher aufwendig“ liegen. Die obersten und unteren Landesbehörden hingegen weisen beide ein Antwortverhalten im Bereich „ziemlich/eher aufwendig“von 70 Prozent und mehr auf. Damit konnte These Nr. 6, mit der die Vermutung formuliert wurde, dass das Tauschverfahren einen Wechsel des Bundeslandes kompliziert mache, bestätigt werden.

Tabelle 4: Tauschpartner zu finden sei aufwendig nach Behördenebene

\begin{tabular}{|l|c|c|c|c|c|}
\hline & $\begin{array}{l}\text { Oberste } \\
\text { Landesbehörde }\end{array}$ & $\begin{array}{l}\text { Landes- } \\
\text { oberbehör- } \\
\text { de }\end{array}$ & $\begin{array}{l}\text { Landesmittel- } \\
\text { behörde }\end{array}$ & $\begin{array}{l}\text { Untere } \\
\text { Landesbehör- } \\
\text { de }\end{array}$ & $\begin{array}{l}\text { Andere Ebe- } \\
\text { ne/ andere } \\
\text { Bezeichnung: }\end{array}$ \\
\hline $\begin{array}{l}\text { ziemlich } \\
\text { aufwendig } \\
\text { eher aufwendig }\end{array}$ & $50,0 \%$ & $11,1 \%$ & $21,1 \%$ & $59,0 \%$ & $31,8 \%$ \\
teils/teils & $20,0 \%$ & $55,6 \%$ & $31,6 \%$ & $15,4 \%$ & $36,4 \%$ \\
eher unaufwendig & $0,0 \%$ & $11,1 \%$ & $36,8 \%$ & $10,3 \%$ & $13,6 \%$ \\
ziemlich unauf- & $0,0 \%$ & $0,0 \%$ & $0,0 \%$ & $0,0 \%$ & $0,0 \%$ \\
wendig & $0,0 \%$ & $11,1 \%$ & $5,3 \%$ & $15,4 \%$ & $18 \%, 2 \%$ \\
weiß nicht & $100,0 \%$ & $100,0 \%$ & $100,0 \%$ & $100,0 \%$ & $100,0 \%$ \\
Gesamt & &
\end{tabular}

Quelle: Eigene Berechnung und Darstellung.

Schließlich wurde im Vorfeld der Umfrage die Hypothese B formuliert, dass die bereits ausgeprägt föderalisierten Bereiche der Polizei und des Lehramts wegen ihrer bereits längeren Erfahrung eine stärker ausgeprägte Wahrnehmung des mit dem Tauschverfahren verbundenen Aufwands haben als dies im Bereich der allgemeinen Verwaltung und der Finanzverwaltung der Fall ist. In Abbildung 11 werden daher die Antworten nach Beamtengruppen aufgeschlüsselt. Demnach wird das Finden eines Tauschpartners vor allem bei den Beamten der allgemeinen Verwaltung (84,6 Prozent) und den Finanzbeamten (82,5 Prozent) als „eher/ziemlich aufwendig“ eingeschätzt. Polizisten (59,4 Prozent) und Lehrer (48,5 Prozent) sehen das Problem zwar auch sehr klar, im Vergleich zu den neu föderalisierten Beamtengruppen jedoch deutlich geringer. Damit wurde Hypothese B nicht bestätigt. 
Abbildung 11: Einschätzung: Finden eines Tauschpartners

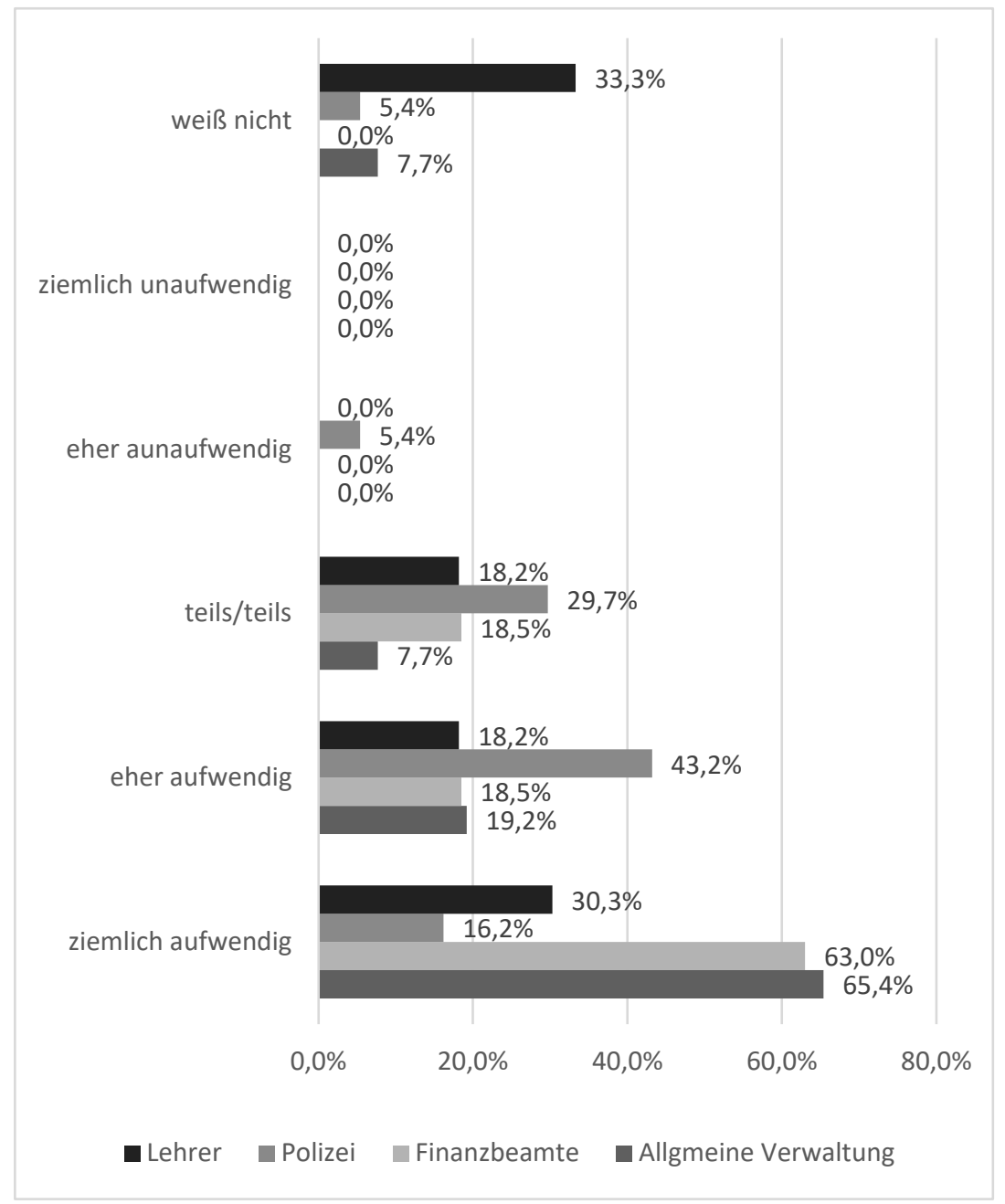

Quelle: Eigene Datenerhebung und eigene Berechnung. 
Eine mögliche Erklärung für dieses unerwartete Ergebnis könnte einerseits sein, dass das Tauschverfahren bei Polizisten und Lehrern mittlerweile bekannt ist und sich neben den offiziellen Vermittlungen eines Tauschpartners über den Versetzungsantrag auch Tauschbörsen über Gewerkschaftszeitungen und Internetseiten etabliert haben. Mit einem Tauschverfahren in der allgemeinen Verwaltung und der Finanzverwaltung konnten hingegen noch keine hinreichenden Erfahrungen gesammelt werden. Die Verunsicherung ist dementsprechend groß. Andererseits wird bei dieser Beamtengruppe häufig gar kein Tauschsystem angewendet. Das hat zur Folge, dass weniger Tauschpartner im System zu finden sind, was im Bedarfsfall das Auffinden eines Tauschpartners in der Tat sehr aufwendig macht.

\subsubsection{Laufbahnsysteme}

Zur Überprüfung der These Nr.3, der zufolge die unterschiedlichen Vorschriften der Bundesländer einen Bundesländer übergreifenden Dienstherrnwechsel verkomplizieren, wurde eine entsprechende Frage in die Online-Umfrage aufgenommen. Von den Befragten sollte der Satz „Die unterschiedlichen Vorschriften der Bundesländer machen einen Dienstwechsel in ein anderes Bundesland ..." auf einer Fünfer-Skala vervollständigt werden. Die Antwortmöglichkeiten reichten von „ziemlich kompliziert“ bis „ziemlich unkompliziert“. Zunächst fiel bei der Auswertung auf, dass je nach Behördenebene die zusätzliche Kategorie ,weiß nicht“ stark unterschiedlich angeklickt wurde: Während von den Befragten aus den obersten Landesbehörden, Landesoberbehörden und Landesmittelbehörden nur 3,4 Prozent bis 5,1 Prozent mit „weiß nicht“ antworteten, haben fast 23 Prozent der Befragten aus den unteren Landesbehörden diese Antwortoption wahrgenommen. Die eigentliche Frage wurde über alle Behördenebenen und Beamtengruppen hinweg verhältnismäßig einheitlich beantwortet, weshalb eine Differenzierung der Darstellung nach Ebenen nicht geboten ist. Jenseits der Einschätzung „weiß nicht“ stellt sich das Ergebnis nicht als starke Unterstützung der formulierten These Nr. 3 dar:

- Dass die unterschiedlichen Vorschriften der Bundesländer einen Dienstherrenwechsel „ziemlich/eher kompliziert“ machen, gaben 34,5 Prozent der Befragten an,

- 34 Prozent antworteten mit „teils/teils“

- und mit „eher/ziemlich unkompliziert“ antworteten ca. 21 Prozent der Befragten.

Allerdings ist davon auszugehen, dass der relativ hohe Anteil an Befragten aus der unteren Verwaltung, die diese Frage nicht zu beantworten vermoch- 
ten, auf eine gewisse Verunsicherung hindeuten. Da Dienstherrenwechsel sich angesichts der gegebenen Unterschiedlichkeit der Vorschriften lediglich für 20 Prozent der Befragten als eher oder ziemlich $u n k o m p l i z i e r t$ darstellen, kann These Nr. 3 als vorläufig bestätigt gelten.

Etwas konkreter wurde auch nach der Bedeutung der unterschiedlichen Laufbahnsysteme gefragt. Zum Verständnis der Relevanz dieser Frage sei nochmals daran erinnert, dass seit der Föderalismusreform I in einigen Bundesländern weiterhin das klassische viergliedrige Laufbahnsystem mit einfachem, mittlerem, gehobenem und höherem Dienst besteht. In anderen Bundesländern wurde der einfache Dienst abgeschafft; dort ist das Laufbahnsystem nun auf drei Laufbahngruppen beschränkt. Wiederum andere Bundesländer haben nur noch die sogenannte Laufbahngruppe 1 mit erstem und zweitem Eingangsamt und die Laufbahngruppe 2 mit wiederum erstem und zweitem Eingangsamt. In zwei Bundesländern ist das Laufbahnsystem auf eine Einheits- bzw. Leistungslaufbahn reduziert, wobei allerdings weiterhin vier unterschiedliche Eingangsämter bestehen. Trotz dieser Vielfalt wurde im Rahmen der Vorschriftenanalyse in allen Bundesländern eine systemimmanente Viergliedrigkeit festgestellt. Die Eingangsvoraussetzungen für die Eingangsämter sind demnach noch immer in allen Bundesländern weitestgehend homogen (Burmester 2015, S. 34 f. sowie weiter oben Kapitel 4.1). Aus diesem Sachverhalt wurde die These Nr. 4 abgeleitet, dass die vordergründig unterschiedlichen Laufbahnsysteme an sich keine nennenswerten Hürden bei einem Bundeslandwechsel darstellen (siehe weiter oben Kapitel 4.5).

Die Ergebnisse der Umfrage können diese These jedoch nicht stützen. Die Einschätzung im Vergleich der Behördenebenen und Beamtengruppen variiert nur marginal; die unterschiedlichen Laufbahnsysteme werden von

- ca. 48 Prozent der Befragten als „ziemlich/eher problematisch“,

- von etwa 28 Prozent der Befragten als „eher/ziemlich unproblematisch“ eingeschätzt und

- von etwa 15 Prozent wurde die Antwortkategorie „teils/teils“ gewählt.

- „Weiß nicht“ antworteten etwa 9 Prozent.

Im Vergleich zur Einschätzung der Wirkung der fragmentierten Vorschriften weichen deutlich weniger Befragte auf die Antworten „teils/teils“ und „weiß nicht" aus, was darauf hindeutet, dass die Laufbahnsysteme wesentlich greifbarer sind als der Bezug auf die abstrakteren Vorschriften. Darüber hinaus zeigen die Ergebnisse, dass von einer Diskrepanz zwischen den rein rechtlichen Möglichkeiten eines Bundeslandwechsels und der Wahrnehmung der tatsächlichen Durchführung in der Praxis auszugehen ist. 


\subsection{Anerkennung von Ausbildung}

Abbildung 12: Ausbildung wird voraussetzungslos anerkannt

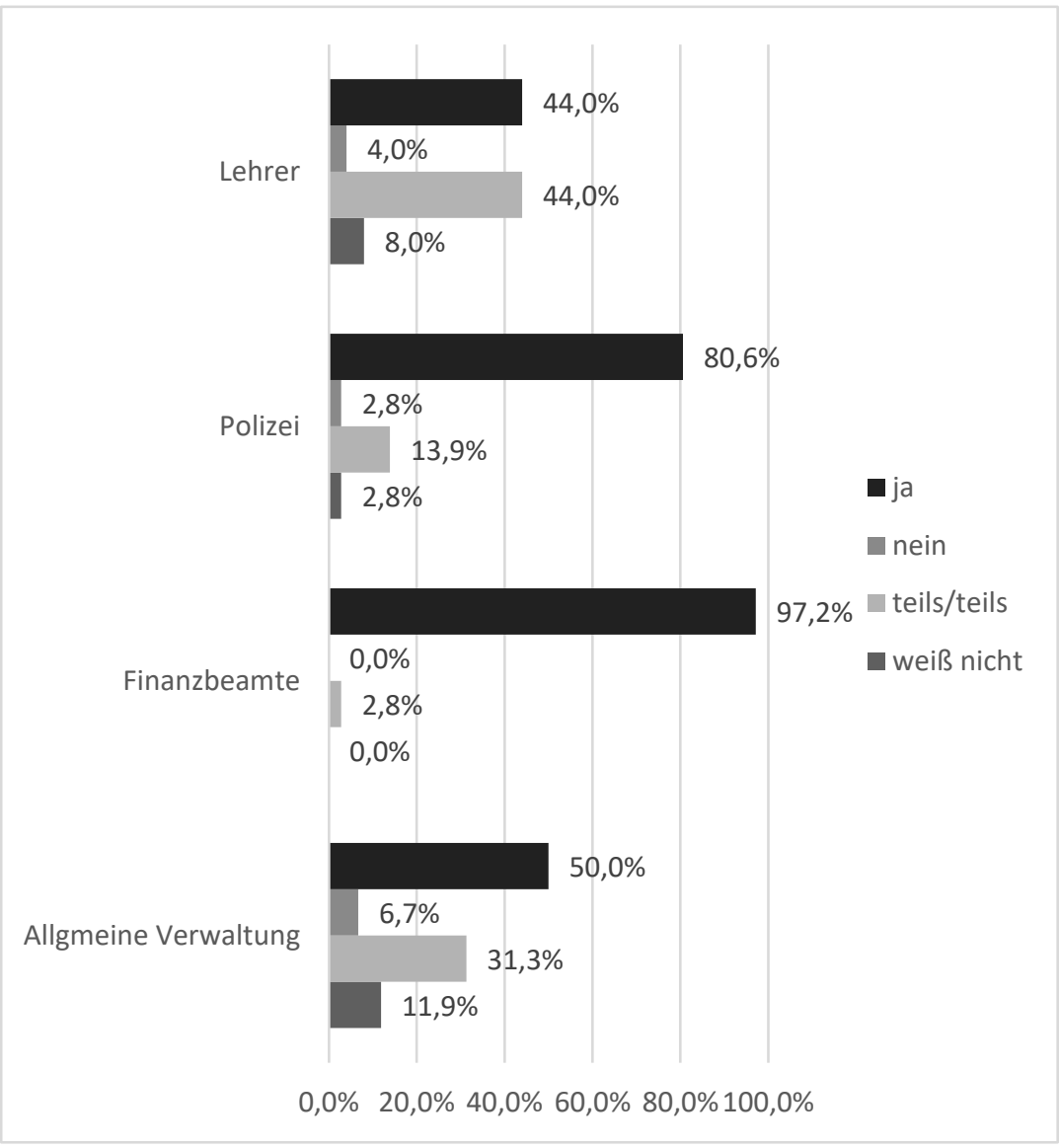

Quelle: Eigene Datenerhebung und eigene Berechnung.

Ein Fragenblock der Online-Befragung hat sich mit der Anerkennung von Ausbildungen und Qualifizierungen im aufnehmenden Bundesland befasst. Dabei gaben 60 bis 70 Prozent der Befragten an, dass Beamte aus einem an- 
deren Bundesland keine Weiterqualifizierungsmaßnahmen, Nachschulungen und Fortbildungen als Einstellungsvoraussetzung durchlaufen müssten. 15 bis 20 Prozent konnten dies nicht einschätzen, sodass nur ein geringer Teil der Befragten angab, dass in ihrem Bundesland die Einstellung an Weiterqualifizierungsmaßnahmen gekoppelt sei. Auf die Frage, wie sich die Häufigkeit der Weiterqualifizierungsmaßnahmen in den letzten fünf Jahren entwickelt habe, war etwa die Hälfte der Teilnehmer der Meinung, dass diese ungefähr gleich oft durchgeführt werden. Weitere 35,4 Prozent antworteten mit „weiß nicht“. Hier scheint sich also vorerst keine bedeutende Veränderung abzuzeichnen.

Es kann auch auf ein Mobilitätshemmnis hindeuten, wenn die Ausbildung eines Beamten nicht uneingeschränkt anerkannt wird. Hierzu wurde insbesondere mit Blick auf den Lehramtsbereich die entsprechende These Nr. 5 formuliert (siehe Kapitel 4.5), die es an dieser Stelle gleichfalls zu überprüfen gilt. In Abbildung 12 werden die Umfrageergebnisse zu diesem Thema grafisch dargestellt. Demnach ist die Anerkennung der Ausbildung vor allem für Finanzbeamte sehr unproblematisch. Auch im Polizeibereich gaben 80,6 Prozent der Befragten an, die Ausbildung werde ohne Weiteres anerkannt. Hier besteht ganz offensichtlich ein hohes Maß an Einheitlichkeit der Ausbildung. Die Antwort „teils/teils“ legt jedoch den Rückschluss nahe, dass zumindest bei einigen wenigen eine Nachschulung, Weiterqualifizierung oder Fortbildungen notwendig war, um im neuen Bundesland ernannt zu werden. Leider liegen für eine Auswertung nach Bundesländern für diese Frage keine ausreichenden Fallzahlen vor, was u.a. auf den insgesamt niedrigen Wert in Höhe von lediglich 2,8 Prozent zurückzuführen ist.

Von beruflichen und wirtschaftlichen Vorteilen geht nach unseren Ergebnissen nur ein geringer Anreiz für einen Bundeslandwechsel aus (siehe Kapitel 5.1). Im Umkehrschluss sollte dies aber nicht zwangsläufig bedeuten, dass diese im Einzelfall nicht zu einem Hemmnis, einer Hürde oder zumindest zu einem Nachteil für Beamte werden können. Durch einen fehlenden qualifikationsgleichen Tauschpartner, eine nicht vorhandene passende Planstelle oder bei ungleicher Zuordnung von Aufgaben zu unterschiedlichen Besoldungsgruppen in den Bundesländern ${ }^{24}$ können bei einem Bundeslandwechsel Nachteile entstehen. Umgangssprachlich wird oft von einer Zurückstufung gesprochen, gemeint ist jedoch die Einstufung eines Beamten aus einem anderen Bundesland in eine niedrigere Besoldungsgruppe. Die Frage, ob eine solche Einstufung in eine niedrigere Besoldungsgruppe im Zuständigkeitsbereich der Befragten vorkommt, bejahten die personal-

24 Zum Beispiel werden Realschullehrer in einigen Bundesländern nach A 12 besoldet, in anderen nach A 13 (siehe Burmester 2015, S. 31 f.). 
verantwortlichen Befragten vor allem im Bereich der Polizei (ca. 62 Prozent) und der Finanzbeamten (ca. 66 Prozent).

Abbildung 13: Einstufung in eine niedrigere Besoldungsgruppe beim Bundeslandwechsel

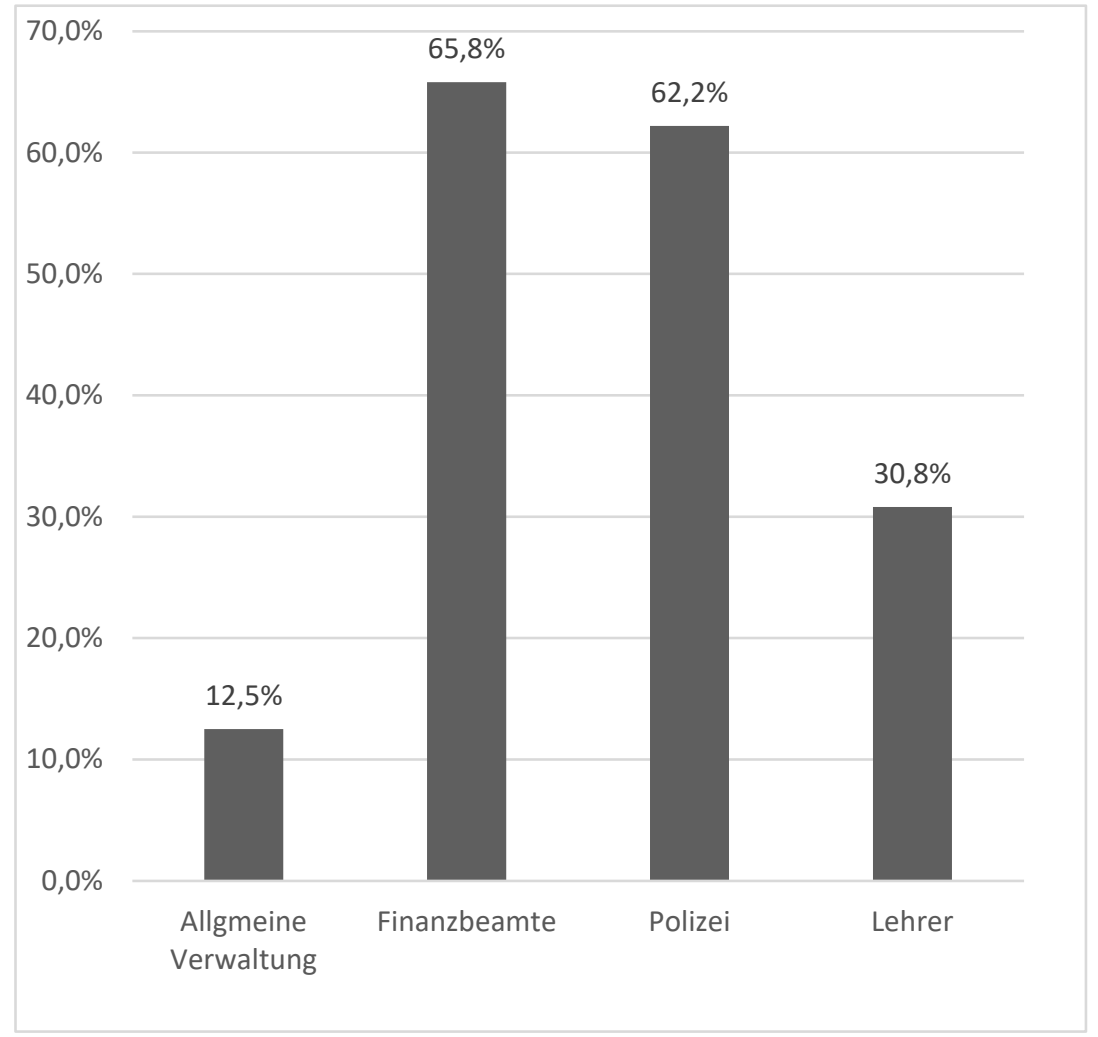

Quelle: Eigene Datenerhebung und eigene Berechnung.

Wie hoch der Anteil der Beamten ist, die nach einem Wechsel in das Bundesland des Befragten in einer niedrigeren Besoldungsgruppe tätig sind, konnten etwa 45 Prozent der Befragten nicht einschätzen. Weitere 43 Prozent schätzten den Anteil auf 10 bis 20 Prozent der neu aufgenommenen Beamten. 
Einer niedrigeren Eingruppierung könnte gegebenenfalls auch mit einer Nachschulung oder Weiterqualifizierung des aufzunehmenden Beamten entgegengewirkt werden. Wenngleich zu Beginn der Befragung die Überlegung implizit eine Rolle spielte, dass die Festlegung solcher Maßnahmen von den Beamten auch als Hürde verstanden werden kann, waren dieser Meinung nur ca. 19 Prozent der Befragten. Hingegen waren 43 Prozent der befragten personalverantwortlichen Mitarbeiter der Meinung, dass die gesetzlichen Vorschriften zu Weiterqualifizierungsmaßnahmen, Nachschulungen und Fortbildungen als Angebot wahrgenommen werden. Dass gut 37 Prozent der Befragten „weiß nicht“ angeklickt haben, macht deutlich, wie wenig eindeutig festgelegt werden kann, ob die Weiterqualifizierungsmaßnahmen als Chance oder Belastung wahrgenommen werden.

\subsection{Wechselprobleme im Zeitverlauf}

Während bislang nach einzelnen als relevant erkannten Aspekten gefragt wurde, erfordert eine zusammenfassende Bewertung der zunehmend fragmentierten Vorschriften eine Perspektive auf potentiell gesteigerte Probleme bei einem Bundesländer übergreifenden Dienstherrenwechsel im Vergleich der letzten Jahre. Daher wurde in der Online-Umfrage nach der Einschätzung der Entwicklungen in den letzten Jahren gefragt. Um in diesem $\mathrm{Zu}-$ sammenhang eine substantielle Zahl von Antwortverweigerungen zu vermeiden und um die Wahl der Antwortkategorie „weiß nicht" so gering wie möglich zu halten, wurde die Föderalismusreform bewusst nicht explizit in der Frage genannt. Auch die Festlegung auf ein bestimmtes Jahr wurde nicht als sinnvoll erachtet, weil die neuen Vorschriften in den verschiedenen Ländern zu sehr unterschiedlichen Zeitpunkten in Kraft getreten sind. Bei der inhaltlichen Vorbereitung der Umfrage wurde die Hypothese $\mathrm{C}$ formuliert, dass in den letzten Jahren die Fragmentierung der Vorschriften vor allem in der allgemeinen Verwaltung zu Problemen geführt habe, da der Polizei- und der Lehramtsbereich bereits vorher föderalen Strukturen unterlagen.

Die Hypothese wird durch die Ergebnisse der Online-Umfrage gestützt. Auf die Fragen, ob in den letzten Jahren vermehrt Probleme bei Bundesländer übergreifenden Dienstherrnwechseln aufgetreten sind, antworteten 
- für die allgemeine Verwaltung 20 Prozent mit „Ja“, 33,3 Prozent mit „Nein“ und 46,7 Prozent mit „weiß nicht“;

- für die Finanzverwaltung 19,4 Prozent mit „Ja“, 50 Prozent mit „Nein“ und 30,6 Prozent mit „weiß nicht“;

- für die Polizei 13,9 Prozent mit „Ja“, 69,4 Prozent mit „Nein“ und 16,7 Prozent mit ,weiß nicht“

- und für die Lehrer nur 4 Prozent mit „Ja“, 68 Prozent mit „Nein“ und 28 Prozent mit „weiß nicht“.

Es zeigt sich also, dass insbesondere für den bereits länger föderalisierten Lehramtsbereich in nur sehr geringem Umfang eine Zunahme von Problemen im Zeitverlauf bei einem Bundesländer übergreifenden Dienstherrnwechsel gesehen wird. Im Polizeibereich ist zwar eine etwas höhere Problemwahrnehmung zu verzeichnen, aber die „Nein“-Stimmen sind sowohl für den Lehramtsbereich als auch für die Polizei mit jeweils fast 70 Prozent sehr hoch. Es sind also weit mehr als die Hälfte der personalverantwortliche Mitarbeiter aus diesen Bereichen der Meinung, dass sich keine Verschlechterung eingestellt habe. Als Grund für die vermehrten Probleme wird im Polizeibereich fast ausschließlich auf das Tauschsystem und das Fehlen eines Tauschpartners verwiesen.

Auch bei den Finanzbeamten und der allgemeinen Verwaltung gibt die Mehrheit der Befragten an, dass es in den letzten Jahren nicht vermehrt zu Problemen gekommen sei, jedoch fallen hier die Werte nicht so deutlich aus wie im Bereich des Lehramts und der Polizei. Auch hier hatten die Befragten in einem offenen Textfeld die Möglichkeit, Gründe für die in ihrer Wahrnehmung zunehmenden Probleme anzugeben. Unter den Befragten der allgemeinen Verwaltung wurde mehrfach genannt,

- dass aufgrund knapper Personalressourcen und fehlender Nachbesetzungsmöglichkeiten - zum Teil bedingt durch den demografischen Wandel - keine Freigabeerklärung erteilt wurde,

- dass die Laufbahnen nicht mehr zusammenpassen und eine Einordnung daher schwierig sei,

- dass die Laufbahnbefähigung und die Weiterqualifizierungen nicht anerkannt werden und

- dass sich die Zuordnung zu den Besoldungsgruppen schwierig gestaltet und es Probleme mit Ausgleichzahlungen bei Besoldungserhöhungen im Herkunftsbundesland sowie mit Sonderzahlungen gibt.

Die personalverantwortlichen Mitarbeiter aus der Finanzverwaltung verwiesen vor allem auf das Fehlen eines geeigneten Tauschpartners. 


\section{Fallstudien}

Die Vorschriftenanalyse bestätigte eine nach der Föderalismusreform I verstärkt einsetzende Fragmentierung des Beamtenrechts. Damit wurde die Vermutung genährt, dass ein Länder-Länder-Wechsel von Beamten sich zunehmend aufwendiger und auch problematischer in den bislang wenig föderalisierten Bereichen der Finanz- und allgemeinen Verwaltung gestaltet. Darüber hinaus konnten durch die Vorschriftenanalyse bereits Teilbereiche konkret benannt werden, in denen es mutmaßlich vermehrt zu Schwierigkeiten kommen kann. Explizit genannt wurden hier u.a. die unterschiedliche Zahl von Laufbahngruppen und die Nichtanerkennung von erfahrenen Weiterbildungen.

In einer sich anschließenden Online-Umfrage unter Personalverantwortlichen konnte nachgefragt werden, ob sich die aus der Vorschriftenanalyse ergebenden mutmaßlichen Probleme aus der Sicht der Befragten tatsächlich eingestellt haben. Darüber hinaus konnte der Frage nachgegangen werden, ob die sich auseinanderentwickelnden Laufbahngesetze in der Vollzugspraxis zu Mobilitätshemmnissen führen. Auch wenn die Daten auf einer individuellen Ebene erhoben wurden, mussten sie für die Auswertung zwangsläufig aggregiert werden. So konnten Probleme zwar festgestellt und auch die Wahrnehmungen der Befragten erfasst werden. Wirkungszusammenhänge lassen sich auf Basis einer solchen Befragung allenfalls vermuten, jedoch nicht konkret nachzeichnen. Hier setzen die Fallstudien an. Sie erlauben wegen der konkreten Rekonstruktion eines Falles die Aufdeckung von Wirkungszusammenhängen. Dank der Erkenntnisse, die sich sowohl aus der Vorschriftenanalyse als auch aus der Online-Umfrage generieren ließen, konnten die Fallstudien bereits sehr informiert geplant und durchgeführt werden. In der hier dokumentierten Studie greifen die verschiedenen methodischen Ansätze also bewusst ineinander.

Im Folgenden wird auf die Ergebnisse eingegangen, die sich aus den Fallstudien extrahieren lassen. Dabei wird darauf verzichtet, den jeweiligen Erkenntnisprozess nachzuzeichnen (siehe Kapitel 2.2). Vielmehr steht die kompakte Darlegung der Ergebnisse im Vordergrund. Dabei wird zunächst auf die Motivation für einen Wechsel eingegangen (Kapitel 6.1). Hieran schließt sich die Darstellung der in dieser Studie in den Mittelpunkt gerückten Probleme bei einem länderübergreifenden Wechsel des Dienstherrn an (Kapitel 6.2). Um eine schärfere analytische Durchdringung zu erreichen, werden die ausgemachten Probleme dabei verschiedenen Kategorien zugeordnet. Es folgen Informationen zur Belastung der Beamten durch die In- 
transparenz der Wechselverfahren und die sich daraus ergebende Ungewissheit (Kapitel 6.3). Abschließend werden Aspekte diskutiert, die für einen Wechsel tendenziell förderlich sind (Kapitel 6.4).

\subsection{Motivation für einen Wechsel}

Wettbewerb zwischen den Bundesländern kann nur entstehen, wenn einerseits die Beamten das Bundesland frei wechseln können und andererseits vom Beamtenrecht zentrale Anreize für einen Wechsel des Bundeslandes ausgehen. Besteht hingegen zwar ein Anreiz für einen Wechsel, fehlt es jedoch an der konkreten Möglichkeit zum Wechsel, wird zwar ein Wanderungsdruck erzeugt, dieser wird sich aber nicht real umsetzen lassen. Bestehen hingegen keine Hürden für einen Wechsel des Bundeslandes, geht aber vom veränderten Beamtenrecht kein Anreiz für einen Wechsel aus, wird eine solche Veränderung des Beamtenrechts dem Bundesland keinen Vorteil im „Wettbewerb um die besten Köpfe“ (Schäuble im DGB-Interview 2006a, S. 1) verschaffen.

Ob ein Anreiz für einen Wechsel besteht, hängt von vielen Faktoren ab, wie z. B. von der jeweiligen Besoldungshöhe oder vom jeweils geltenden Beamtenrecht. $\mathrm{Ob}$ vom Beamtenrecht tatsächlich ein zentraler Anreiz bei der Auswahl des Bundeslandes für das eigene Dienstverhältnis ausgeht, wurden die Befragten im Rahmen der Erfassung ihrer Motivation für den Wechsel gefragt. Die nachfolgenden Ergebnisse haben keinen repräsentativen Charakter, vervollständigen jedoch das bisher entstandene Bild über die Motivationsgrundlagen der wechselwilligen Beamten.

In 84 Prozent der Fälle (in absoluten Zahlen: in 27 von 32 Fällen) wurden für den Grund des Wechsels persönliche oder familiäre Gründe genannt. So sollte beispielsweise eine bestehende Fernbeziehung zu einem Partner beendet werden. In mehreren Fällen bestand auch die Verpflichtung oder der Wunsch die eigenen Eltern im Wunschbundesland zu pflegen. Es findet sich auch schlicht der Wunsch, in die alte Heimat zurückzuziehen. So gab es mehrere Fälle, in denen ein Beamter in einem Nachbarbundesland die Ausbildung absolviert hatte, nachdem er in seinem Heimatbundesland keine Zusage bekommen hatte. Nach der Ausbildung bestand dann der Wunsch, wieder zurück in das Heimatbundesland zu wechseln.

In ca. 16 Prozent aller Fälle (in absoluten Zahlen: in fünf von 32 Fällen) wurden dienstliche Gründe oder der Wunsch nach beruflicher Weiterentwicklung geäußert. Hinter den dienstlichen Gründen verbergen sich häufig Unzufriedenheiten mit der lokalen Dienststelle oder auch Fälle von Mobbing. Der Wunsch nach beruflicher Weiterentwicklung wurde beim Fehlen 
von Beförderungsmöglichkeiten im Ausgangsbundesland genannt oder es wurden reizvollere Aufgaben im neuen Bundesland gesehen. Der Anreiz zu wechseln war in diesen Fällen mit der konkreten potentiellen Aufgabe beim neuen Dienstherrn verbunden und nicht mit einer aus dem Beamtenrecht resultierenden Attraktivität.

Aufgeteilt auf die einzelnen Beamtengruppen strebten in 50 Prozent der Fälle (in absoluten Zahlen: in drei von sechs Fällen) die Beamten der allgemeinen Verwaltung aus persönlichen/familiären Gründen den Wechsel an, während die andere Hälfte der Beamten aus dienstlichen Gründen wechseln wollte. Dabei hatten zwei von drei Beamten ihr Amt im höheren Dienst.

Bei der Polizei waren bei 95 Prozent aller Fälle (in absoluten Zahlen: in 19 von 20 Fällen) private oder familiäre Gründe für den angestrebten oder durchgeführten Wechsel ausschlaggebend. Allein bei 5 Prozent der Fälle (in absoluten Zahlen: in einem Fall) war die berufliche Weiterentwicklung der Grund für das Bestreben, das Bundesland zu wechseln. Der betroffene Beamte beklagte die fehlenden Beförderungsstellen in seinem Bundesland; er sah deshalb keine Möglichkeiten Karriere zu machen. Folglich bestehen für alle betrachteten Fälle im Polizeibereich wichtige, nicht monetäre Gründe für einen Wechsel. Den Beamten geht es also nicht schlicht um einen Wechsel, um eine höhere Besoldung im neuen Bundesland zu realisieren, sondern um Familienzusammenführung und die Abwendung persönlicher Härten.

Im Lehramtsbereich gaben die Beamten in 83 Prozent der Fälle an, aus persönlichen und familiären Gründen wechseln zu wollen. Nur in ca. 17 Prozent der Fälle (in absoluten Zahlen: in einem Fall) wurde angegeben, dass der Wechsel aus dienstlichen Gründen angestrebt bzw. vollzogen wurde. Der Beamte wurde auf seiner alten Dienststelle gemobbt.

Bis auf die allgemeine Verwaltung, bei der vor allem im höheren Dienst aus beruflichen Gründen gewechselt wird, lässt sich keine besondere Häufung in den Laufbahngruppen feststellen.

In keinem Fall wurde der Wechsel mit einem attraktiveren Beamtenrecht im neuen Bundesland begründet. Auch die unterschiedliche Besoldung spielte keine Rolle, im Gegenteil: Aus familiären Gründen wechselnde Beamte tun dies auch, wenn das Grundgehalt im neuen Bundesland niedriger ausfällt als im alten oder sich ihre Nettobezüge durch Wegfall beispielsweise der (freien) Heilfürsorge oder einer ungünstigeren Berechnung der Erfahrungsstufe verschlechterte. Häufig haben sich die Beamten im Vorfeld des Wechsels auch gar nicht über die Höhe der Besoldung am neuen Dienstort informiert, was weiter dafür spricht, dass die Besoldungshöhe kein dominanter Faktor für oder gegen einen Wechsel ist. Denkbar ist allerdings auch, dass die Beamten trotz aller Informationspolitik des DGB noch nicht reali- 
siert haben, dass die Bundesländer mittlerweile stark unterschiedlich besolden.

Insgesamt kann auf Basis der Fallstudien davon ausgegangen werden, dass zumindest der Anreiz eines attraktiven Beamtenrechts, sollte er bestehen, von anderen dominanteren Faktoren überlagert wird. Eine Abwanderung des Personals in Bundesländer mit relativ hoher Besoldung oder anderen beamtenrechtlichen Vorteilen ist nicht zu beobachten. Diese Beobachtung wurde auch durch die Interviews mit Gewerkschaftsvertretern bestätigt.

\subsection{Probleme bei einem Länder-Länder-Wechsel}

Es bestehen unterschiedliche Probleme bei einem Länderwechsel. Diese lassen sich in zwei Hauptkategorien einteilen. Erstens bestehen Probleme, die durch unterschiedliche Landesgegebenheiten verursacht werden. Diese Probleme resultieren hauptsächlich aus dem sich auseinanderentwickelnden landesspezifischen Bestand des Laufbahnrechts. In dieser Kategorie können die Probleme unterschiedlich ausgeprägt sein. Das heißt, es gibt harte Probleme, welche einen Wechsel verhindern oder zumindest massiv erschweren, aber auch weichere Probleme, die den Wechsel nicht unbedingt erschweren, jedoch den wechselnden Beamten benachteiligen. Eine eindeutige Zuordnung zu diesen beiden Kategorien ist jedoch nicht möglich. Denn als wie hart oder weich ein Problem empfunden wird, hat vor allem mit der Prioritätensetzung des einzelnen Beamten zu tun. Die Begriffe „hart“ und „weich“ sollen daher als Extrempunkte eines Kontinuums verstanden werden. Die Einordnung einer spezifischen Situation auf diesem Kontinuum wird durch verschiedene Beamte unterschiedlich ausfallen. So kann beispielsweise eine geringere Besoldung für einen Beamten ein weicheres Problem darstellen, wenn der Wechsel ermöglicht, weniger zu pendeln und damit mehr Zeit für die Familie zu haben. Ein anderer Beamter, der möglicherweise größere finanzielle Verpflichtungen eingegangen ist, kann die niedrigere Besoldungshöhe im Vergleich zu einem langen Arbeitsweg als gravierenderes Problem empfinden.

Zweitens gibt es Probleme, die erst durch das für einen Wechsel vorgesehene Verfahren entstehen. Diese Probleme resultieren weniger aus der Unterschiedlichkeit des Laufbahnrechts, sondern aus dem organisatorischen Ablauf des Wechsels zwischen den Ländern. Im Folgenden werden die Probleme, welche im Rahmen der Durchführung der Fallstudien herausgearbeitet werden konnten, dargestellt und in einer der beiden Problemkategorien verortet. 


\subsubsection{Probleme aus Landesgegebenheiten}

Im Folgenden sollen zunächst die Probleme diskutiert werden, die über alle untersuchten Beamtengruppen hinweg Gültigkeit beanspruchen können. Anschließend richtet sich die Analyse auf diejenigen Probleme, die speziell für bestimmte Beamtengruppen gelten. Als Problem wird Beamtengruppen übergreifend die unterschiedliche Besoldungshöhe wahrgenommen. Die Unterschiede können auf mehrere Teilaspekte zurückgeführt werden: Erstens ist das voneinander abweichende Grundgehalt zu nennen sowie zweitens eine unterschiedliche Praxis bei der Zuordnung zu den verschiedenen Erfahrungsstufen, was einen merklichen Effekt auf die letztendliche Besoldungshöhe hat. Jedes Bundesland entscheidet autonom, welche Zeiten für die Berechnung der Erfahrungsstufen anerkannt werden. So kann ein Beamter in eine niedrigere Erfahrungsstufe eingestuft werden, wenn im aufnehmenden Bundesland beispielsweise Wehrdienstzeiten, Ausbildungszeiten oder auch Zeiten für politische Mandate nicht anerkannt werden, dies jedoch der Fall im abgebenden Bundesland war. Auch wurde häufig berichtet, dass die Berechnung dieser Zeiten mitunter langwierig ist. Teilweise haben die betroffenen Beamten bis zur erfolgten Nachzahlung, die erst ein Jahr nach dem Wechsel erfolgte, jeden Monat auf mehrere hundert Euro verzichten müssen. Auch wenn die Beamten ganz offensichtlich nicht einen Wechselwunsch wegen einer möglicherweise höheren Besoldung im aufnehmenden Bundesland entwickelt haben, so empfinden sie es jedoch als ungerecht, wenn sie im aufnehmenden Bundesland vorübergehend oder auf Dauer auf Teile der bislang erfahrenen Besoldung verzichten müssen.

Daneben können die verschiedenen Systeme zur Absicherung im Krankheitsfall für alle Beamtengruppen ein Problem darstellen. Der Nachteil wird von den Beamten jedoch stark unterschiedlich wahrgenommen. So wurde in manchen Fällen der Wechsel von der (freien) Heilfürsorge zu einem Bundesland mit einem Beihilfesystem von manchen Beamten als negativ empfunden, weil sie zusätzliche finanzielle Aufwendungen für eine die Beihilfe ergänzende Krankenversicherung haben. Gleichzeitig gab es Fälle, bei denen der Wechsel von einem Bundesland mit Beihilfe in ein Bundesland mit (freier) Heilfürsorge ebenso als negativ empfunden wurde, da die als vorteilhaft betrachtete private Krankenversicherung verloren ging.

Ein weiteres Beamtengruppen übergreifendes Hindernis sind die verschiedenen Höchstaltersgrenzen für die Verbeamtung. Die Höhe der Altersgrenze ist in den Bundesländern unterschiedlich geregelt (siehe Burmester 2015, S. 47); dies gilt auch für Ausnahmen. In manchen Bundesländern kann bei Überschreitung der Höchstaltersgrenze eine übergeordnete Stelle, wie etwa die oberste Dienstbehörde, der Finanzminister, der Innenminister, 
der Landespersonalausschuss oder ein Gremium aus diesen darüber bestimmen, ob ein Bewerber trotz Überschreitung der Höchstaltersgrenze verbeamtet werden kann. Des Weiteren gibt es in einigen Bundesländern Anrechnungstatbestände, wie Kinderbetreuung oder die Ableistung eines freiwilligen sozialen Jahres, welche die Höchstaltersgrenze weiter verschieben. Dann wiederum gibt es Bundesländer, die gesetzlich bestimmen, dass die Höchstaltersgrenze nicht für Bewerber gilt, die von einem anderen Dienstherrn auBerhalb des Geltungsbereichs des heimischen Beamtengesetzes kommen, ihre Pensionslasten jedoch nach dem Pensionslastenteilungsstaatsvertrag aufgeteilt werden.

Im Rahmen der Fallstudien konnten bei der allgemeinen Verwaltung keine Probleme identifiziert werden, die über die gruppenübergreifenden Probleme hinausgehen.

Insbesondere bei der Polizei sind Probleme durch eine nur teilweise Anerkennung der Laufbahnbefähigung aufgetreten. Dies ist nicht zu verwechseln mit dem Problem einer vollständigen Nichtanerkennung einer Laufbahnbefähigung, bei der ein Wechsel nicht möglich ist. Durch die Teilanerkennung haben Beamte Nachteile bei der weiteren Karriere im neuen Bundesland. Beamte, die beispielsweise in Mecklenburg-Vorpommern nach $\mathbb{1 0}$ LVOPol M-V (mittlerweile durch PolLaufbVO M-V ersetzt) aufgestiegen sind, haben Probleme, diesen Aufstieg in anderen Bundesländern voll anerkannt zu bekommen. In der Praxis bedeutet dies, dass sie keine vollwertige Laufbahnbefähigung haben und in den Karrieremöglichkeiten innerhalb ihrer Laufbahn nach oben beschränkt sind, ähnlich wie bei einem Verwendungsaufstieg. Gleichzeitig erkennt aber auch Mecklenburg-Vorpommern bestimmte Aufstiegsarten nur zum Teil an. Beamte, die in anderen Bundesländern einen Aufstieg in den gehobenen Dienst vollzogen haben, bei welchem ein Studium mit Abschlussarbeit nicht erforderlich ist, haben laut Aussage mehrerer Wechsler mit diesem Aufstieg im gehobenen Dienst nur die Möglichkeit, die Laufbahngruppe bis zur Besoldungsgruppe A 11 und nicht bis A 13 zu durchlaufen.

Des Weiteren wurde bei der Polizei beobachtet, dass ein Wechsel in manchen Fällen nur möglich war, wenn der betroffene Beamte sich in ein niedrigeres Amt mit einer niedrigeren Besoldungsgruppe hat einordnen lassen. So wurde berichtet, dass ein Tausch eines Hauptkommissars mit einem Oberkommissar nur möglich war, wenn sich der Hauptkommissar als Oberkommissar übernehmen lassen würde.

Auch gibt es immer wieder Fälle, in denen ein Beamter nur in einer vergleichsweise niedrigen Laufbahngruppe im aufnehmenden Bundesland übernommen wird. So werden Kommissare beispielsweise nur als Hauptmeister übernommen. Begründet wird dies informell mit dem Argument, 
dass jeder neu eingestellte Kommissar einem heimischen Hauptmeister die Aufstiegsmöglichkeit nimmt.

Auch gab es häufig Probleme mit der Gesundheitsprüfung, welche bei einem Bundeslandwechsel im Polizeidienst erneut abgelegt werden muss. So wird beispielsweise in den Bundesländern die Dienstvorschrift PDV 300, welche bundeseinheitlich die Anforderungen der gesundheitlichen Polizeidienstfähigkeit regelt, unterschiedlich hart ausgelegt. So kam es mehrfach vor, dass ein in einem Bundesland als diensttauglich attestierter Beamter in einem anderen Bundesland als dienstuntauglich eingestuft wurde.

Auch wird in den Bundesländern verschiedenartig mit dienstlich erworbenen Qualifikationen umgegangen. So wurden in einigen Fällen in anderen Bundesländern ausgestellte, grundlegende Bescheinigungen über den Umgang mit der Dienstwaffe oder der Berechtigungsschein für das Führen des Dienstfahrzeuges nicht anerkannt. Die gewechselten Beamten mussten mit großem Aufwand abgelegte Prüfungen und Schulungen erneut ablegen bzw. durchlaufen.

Ein weiteres Problem stellen die unterschiedlichen Reformen des Laufbahnrechts da, womit konkret die Laufbahngruppen angesprochen sind. Zwar sind in allen Ländern, wie die Vorschriftenanalyse gezeigt hat, die Strukturen bei näherer Betrachtung ähnlich geblieben, jedoch ist die Einstellungspraxis in diese Laufbahnen in den Bundesländern sehr unterschiedlich. So bilden Nordrhein-Westfalen, Niedersachsen, Bremen, RheinlandPfalz, das Saarland und Hessen nicht mehr im mittleren Polizeidienst aus und stellen darüber hinaus auch keine Polizisten mehr in diesen ein. Somit können Polizisten aus dem mittleren Dienst ohne einen Aufstieg nicht in die genannten Bundesländer wechseln.

Anders als bei den Polizeibeamten kann die Ausbildung im Lehramtsbereich durch die zahlreichen unterschiedlichen Studiengänge und Fächerkombinationen ein Problem bei einem Wechsel darstellen. Da zurzeit eine große Nachfrage nach Lehrkräften besteht, wird mit den Unterschiedlichkeiten der Ausbildung sehr pragmatisch und flexibel umgegangen. Trotzdem gibt es Ausbildungsarten, die den Wechsel in bestimmte Bundesländer ausschließen. Beispielsweise können Sonderschullehrer mit nur einem Fach nicht in den Berliner Schuldienst übernommen werden. 


\subsubsection{Probleme durch das Wechselverfahren}

Probleme durch das Wechselverfahren sind bei einer rein juristisch formalen Betrachtungsweise nicht ohne Weiteres zu erkennen. Da ausschließlich der $\$ 15$ BeamtStG Gesetzescharakter besitzt, sind alle Vereinbarungen zwischen den Ländern rein rechtlich nachrangig. In der Praxis werden Länder-LänderWechsel jedoch auch durch Länder-Länder-Vereinbarungen geregelt, welche eine zentrale Bedeutung aufweisen. So gibt es KMK- und IMK-Beschlüsse, welche die Bedingungen bei einem Wechsel nach $₫ 15$ BeamtStG regeln. Sie sind jedoch nicht immer funktional.

Für die drei Beamtengruppen gelten unterschiedliche Verfahren für den Bundesländergrenzen übergreifenden Wechsel der Behörde. Bei der allgemeinen Verwaltung dominiert das Prinzip „Bewerbung auf offene Stelle“. Bei der Polizei herrscht das Tauschverfahren vor, während bei den Lehrern beide Verfahren zur Anwendung kommen. Erstens gibt es die Möglichkeit, sich auf eine freie Stelle zu bewerben. In diesem Fall kommt die sogenannte Nachsteuerung zum Tragen. Der abgebende Dienstherr ist angehalten, die Freigabe zügig zu erteilen. Er kann jedoch Ablehnungsgründe geltend machen. Zweitens wurde ein institutionalisiertes Tauschverfahren etabliert, was sich jedoch vom Kopf-zu-Kopf-Tauschverfahren, wie es bei den Polizeibeamten durchgeführt wird, deutlich unterscheidet. Im Folgenden werden die verschiedenen Verfahren und die jeweils bei ihnen auftretenden Probleme dargelegt und diskutiert.

\subsubsection{Wechselregime bei der allgemeinen Verwaltung}

Das Wechselverfahren bei der allgemeinen Verwaltung entspricht häufig dem in der Privatwirtschaft. Wenn eine Stelle neu zu besetzen ist, wird diese in der Regel zuerst intern ausgeschrieben. Ist sie dann nicht zu besetzen, wird die Ausschreibung auf externe Bewerber ausgeweitet. Ein Wechselwilliger bewirbt sich beim ausschreibenden Bundesland auf diese Stelle und wird bei Eignung auch versetzt. Die dafür erforderliche Freigabe beim abgebenden Dienstherrn wird in der Regel erteilt. Ein Tauschverfahren, bei dem der abgebende Dienstherr auf einen gleichwertigen Ersatz besteht, ist unbekannt. Es gibt sehr vereinzelt Tauschbörsen. Diese dienen aber nicht dem Zweck, dem angebenden Dienstherrn Ersatz zu stellen, sondern dem Wechselwilligen bei einem Mangel an freien Stellen eine Stelle durch einen Tausch zu beschaffen.

Die entsprechenden Verfahren verlaufen weitgehend problemlos. Zwar wird die Freigabe vom abgebenden Dienstherrn in manchen Fällen etwas verzögert, jedoch nur mit dem Ziel, den jeweiligen Beamten begonnene 
Aufgaben abschließen zu lassen. In der Praxis erfolgt häufig auch nicht die sofortige Versetzung, sondern der Beamte wird zuerst mit dem Ziel einer Versetzung abgeordnet. Das hat für beide Seiten den Vorteil einer Probezeit. Der Dienstherr kann sich von der Leistung des neuen Beamten überzeugen, während der Beamte die neue Dienststelle kennenlernen kann.

In manchen Fällen werden durch dieses Ausschreibungs- und Bewerbungsverfahren Wechsel erschwert, nämlich dann, wenn keine freien Stellen vorhanden sind. Da die Kultur eines Tausches unterentwickelt ist, können betroffene Beamte einen angestrebten Wechsel häufig nicht oder erst deutlich verspätet realisieren.

\subsubsection{Wechselregime bei der Polizei}

Bei der Polizei gilt das sogenannte Kopf-zu-Kopf-Tauschverfahren. Damit ist gemeint, dass ein Polizeibeamter nur in ein anderes Bundesland wechseln darf, wenn er einen gleichwertigen Tauschpartner aus einem anderen Bundesland vorweisen kann.

Die Details zum Tauschverfahren bei der Polizei wurden in einer Absprache der Innenministerkonferenz festgelegt. Es sind insgesamt drei verschiedene Wege möglich: Erstens, ein Beamter stellt einen Antrag auf Versetzung in ein anderes Bundesland. Die jeweils zuständige Stelle im abgebenden Bundesland ermittelt, ob Wechselwillige aus dem anderen Bundesland einen Antrag auf Versetzung in das eigene Bundesland gestellt haben. Wenn das der Fall ist und diese Person als gleichrangig eingestuft wird, kann der Wechsel vollzogen werden.

Zweitens besteht die Möglichkeit, sich einen Tauschpartner privat zu organisieren. Hierzu finden sich im Internet Tauschbörsen, in denen Beamte sich als wechselwillig eintragen können. Dort kann auch ohne den Dienstherrn vorab zu informieren ein Tauschpartner gesucht und erst anschließend der Antrag auf Versetzung gestellt werden. Die Wechselwilligen nennen jeweils den anderen als Tauschpartner und der Tausch kann im Prinzip vollzogen werden.

Die dritte Möglichkeit ist die des Ringtausches. Dabei sind nicht nur zwei Bundesländer beteiligt, sondern mindestens drei. Ein Beamter, der beispielsweise von Hessen nach Nordrhein-Westfalen wechseln will, tauscht mit einem Beamten, der von Nordrhein-Westfalen nach Mecklenburg-Vorpommern wechseln will, der wiederum mit einem Beamten tauscht, der von Mecklenburg-Vorpommern nach Hessen will. Es liegt auf der Hand, dass dieses Verfahren vergleichsweise aufwendig ist. Auch bedarf es drei gleichwertiger Polizeibeamten, um den Ringtausch zu realisieren. In der Innenmi- 
nisterkonferenz wurden allerdings Ausnahmen für Härtefälle beschlossen. Wie diese Entscheidung aber umgesetzt wird, ist im Augenblick unklar.

In der Praxis treten bei diesem Wechselregime beachtliche Probleme auf; diese betreffen alle drei Möglichkeiten. So können Wechsel, bei denen die Tauschpartner über den Dienstherrn organisiert werden, zu Härten führen. Denn einige Bundesländer gehen nach dem First-come-first-serve-Prinzip vor. So wurde einem Wechsler in Mecklenburg-Vorpommern erklärt, dass auf soziale Gründe keine Rücksicht genommen werden könne. Andere Bundesländer, wie Schleswig-Holstein, haben zumindest für die interne Umsetzung einen Kriterienkatalog, mit denen die Wechselwilligen anhand der Dringlichkeit des Wechselwunsches in eine Rangfolge gebracht werden.

Ein Wechsel kann von der Antragstellung bis zum tatsächlichen Vollzug mehrere Jahre dauern. Hinzu kommt, dass die offizielle Offenlegung eines Wechselwunsches auf der Dienststelle zu Nachteilen führt. Dieser Effekt wurde während der geführten Interviews mehrfach bestätigt. So werden kontingentierte Fortbildungsmaßnahmen, wie z. B. Sporttrainerscheine von der Dienststelle zu allererst an diejenigen Beamten vergeben, die weiterhin bei der Dienststelle bleiben. Ebenso werden wechselwillige Beamte bei Beförderungen übergangen. Dies geht dann auch einher mit Nachteilen bei der dienstlichen Bewertung. Ein solches Verhalten ist aus der Perspektive der Dienstelle ein Stück weit folgerichtig, da diese vor allem die Entwicklung der längerfristig tätigen Mitarbeiter im Blick hat. Auch für den Beamten wäre eine nur sehr kurzfristige Benachteiligung an sich nicht problematisch, weil dieser dann auf der neuen Dienststelle wieder in die Personalentwicklung integriert werden würde. Dauert aber ein Verfahren mehrere Jahre bis vielleicht einem Jahrzehnt, ist der Beamte die ganze Zeit über von der allgemeinen Personalentwicklung abgehängt gewesen. Außerdem geht bei einem Wechsel die Benachteiligung auf der neuen Dienststelle erst einmal weiter. Dem neuen Dienstherrn liegen mit der Übergabe der Personalakte vergleichsweise schlechte Beurteilungen vor und jene werden auch als Grundlage für kommende Beförderungen herangezogen. Da nur zu bestimmten Zeitpunkten beurteilt wird und eine bestimmte Anzahl von Beurteilungen mit in die Entscheidung über Beförderungen einfließt, kann der Beamte mehrere Jahre warten müssen, bis er einen Beurteilungsstand erreicht hat, als wäre er schon immer bei dem neuen Dienstherrn gewesen. Angesichts dieser gravierenden Nachteile verzichten viele Beamte auf eine offizielle Antragstellung, bis sie einen selbstorganisierten Tauschpartner vorweisen können. Dies wiederum ist für das Gesamtsystem dysfunktional, weil damit den Dienstherren weniger Tauschpartner bekannt als tatsächlich vorhanden sind. 
Da in der Praxis ein Tausch in der Regel ohne Tauschpartner nicht möglich ist und es als sehr schwierig empfunden wird, einen passenden Tauschpartner zu finden, werden diese als ein hohes Gut gehandelt. Zusammen mit dem Druck vieler Beamte das Bundesland wechseln zu müssen, um beispielsweise kranke Eltern zu pflegen oder um mehr Zeit mit dem Partner verbringen zu können, entwickelt sich eine ausgeprägte Ellenbogenmentalität, was das System weiter verkompliziert. So ist in vielen Fällen der angestrebte Wechsel daran gescheitert, dass ein Tauschpartner seine Zusage zurückgezogen hat. Häufig hatte das den Grund, dass ein wechselwilliger Beamter sich mehreren Tauschpartnern zum Tausch anbietet, um die Gefahr des Scheiterns zu verringern. Da jedoch nur einer der Tauschpartner zu Zuge kommen kann, gehen die übrigen, lediglich potenziellen Tauschpartner leer aus, was innerhalb des Systems weiteres Misstrauen erzeugt.

Schließlich existiert ein Schwarzmarkt für Tauschpartner. Wegen des Mangels an Tauschpartnern lassen sich Beamte bei einem Wechsel aus einem Bundesland, das nur wenige Wechselwillige aufweist, für ihre Bereitschaft zu wechseln bezahlen. Den Zuschlag erhält in der Regel derjenige, der bereit ist, den höchsten Geldbetrag einzubringen. Dieser firmiert häufig euphemistisch unter dem Begriff der „Umzugskostenpauschale“. Manche Bundesländer haben dieses Problem erkannt und behalten sich vor, den beim Versetzungsverfahren angegebenen Tauschpartner für einen anderen Tausch zu verwenden. Diese Gegenstrategie verursacht aber neue Probleme und Ungerechtigkeiten. So ist es in mehreren Fällen vorgekommen, dass ein wechselwilliger Beamter über mehrere Jahre hinweg mehrere Tauschpartner für seinen Tausch benannt hat, ihm jedoch mitgeteilt wurde, dass sein Wechsel nicht durchgeführt werden könne, weil der Tauschpartner für jemand anderen verwendet werde.

Die Bundesländer gehen mit dem Tauschprinzip auch unterschiedlich um. Es gibt Bundesländer, die dieses hart umsetzen und für jeden Beamten, der geht, einen neuen erwarten, sowie für jeden aufgenommenen Beamten einen ihrer Beamten abgeben wollen. Daneben gibt es Bundesländer, die flexibler agieren. Diese nehmen auch Beamte auf, ohne zwingend einen Tauschpartner abgeben zu wollen oder erteilen Beamten eine Freigabe ohne zwingend einen Ersatz zu erwarten. Dies hat häufig auch mit der Personalpolitik des jeweiligen Landes zu tun. Aus ihr resultieren weitere Probleme. So kam es vor, dass ein Beamter aus einem Bundesland, in dem Stellen abgebaut werden sollen, in ein Bundesland wechseln wollte, indem die Anzahl der Stellen konstant gehalten werden sollte. Das abgebende Bundesland verweigerte die Aufnahme eines Tauschpartners, um die Stelle einsparen zu können. Das aufnehmende Bundesland hatte jedoch die Abgabe eines Beamten für die Aufnahme des neuen Beamten zur Bedingung gemacht, um 
die Zahl der besetzten Stellen konstant zu halten. Folglich scheiterte der Wechsel. Aktuell dürfte das letztgenannte Problem angesichts der zahlreichen unbesetzten Stellen allerdings kaum zum Tragen kommen.

\subsubsection{Wechselregime bei den verbeamteten Lehrkräften}

Das Wechselverfahren im Lehramtsbereich ruht auf zwei Säulen. Die erste Säule ist durch ein reguläres Bewerbungsverfahren markiert. Die zweite Säule besteht aus einem institutionalisierten Lehrertauschverfahren. Die Kultusministerkonferenz wollte mit der Etablierung dieser zweiten Säule sozialen Gesichtspunkten wie der Familienzusammenführung auf besondere Weise Rechnung tragen, wobei bereits bei der ersten Säule die Familienzusammenführung eine wichtige Rolle spielt (KMK, 2001, S. 2). Generell ist der länderübergreifende Wechsel in dieser Beamtengruppe am stärksten verregelt. So sind zum Verfahren gleich mehrere KMK-Beschlüsse gefasst worden.

Bei einem Wechsel im regulären Bewerbungsverfahren steht es Lehrkräften frei, sich auf offene Stellen in anderen Bundesländern zu bewerben. Generell ist dabei der Anspruch der Schülerinnen auf Unterrichtskontinuität zu beachten (KMK, 2001, S. 2). Die sich bewerbenden Lehrer sind verpflichtet, der Bewerbung eine Freigabeerklärung ihrer Dienststelle beizulegen. Diese Freigabeerklärung soll von der bestehenden Dienststelle so großzügig wie möglich unter Beachtung dienstlicher Interessen erteilt werden. Sollten dienstliche Gründe gegen die unmittelbare Freigabe sprechen, wie beispielsweise eine schulspezifische Mangelsituation oder eine Einbindung in abiturvorbereitende Kurse, ist die Freigabe in der Regel nicht später als zwei Jahre nach der Antragstellung zu erteilen (KMK 2001, S. 2).

Das institutionalisierte Lehrertauschverfahren, offiziell als „Einigungsverfahren" (KMK 2001, S. 2) bezeichnet, wird einmal im Jahr zum Schuljahresbeginn mit allen Ländern durchgeführt. Besteht ein besonderer Bedarf, sieht die Vereinbarung ein zweites Verfahren zum Schulhalbjahresbeginn vor. Grundsätzlich besteht die Vereinbarung, dass jedes Bundesland so viele Lehrer aufnehmen soll, wie es an andere Länder abgeben kann (KMK 2002, S. 3).

Für die Antragsstellung gibt es ein einheitliches Formular, welches mindestens sechs Monate vor dem beantragten Wechseltermin bei der zuständigen, d.h. abgebenden Behörde vorliegen muss. Der Austausch selbst wird Ende März/Anfang April und Ende September/Anfang Oktober in einer Sitzung der Länder beschlossen. Der abgebende Dienstherr informiert den Beamten über das Ergebnis der Sitzung. Zieht eine Lehrkraft ihren Antrag auf Wechsel, der bereits vereinbart wurde, zurück, sollen die übrigen vereinbarten Wechsel trotzdem stattfinden (KMK 2002, S. 3 f.). 
Generell soll für die Versetzungen zum Schuljahresbeginn der erste August und zum Halbschuljahresbeginn der erste Februar angestrebt werden. Im Einvernehmen können zwischen den Bundesländern jedoch auch Versetzungen an abweichenden Terminen vereinbart werden (KMK 2002, S.4). Der erforderliche Arbeitsaufwand wird durch ein einheitliches EDV-System versucht zu minimieren (KMK 2001, S. 3).

Der tatsächliche Wechsel bei Lehrkräften zeigte sich nach den in den Fallstudien dokumentierten Erfahrungen als weitestgehend unproblematisch. Zur Zeit der Befragung liefen die meisten Wechsel über die sogenannte Nachsteuerung, das heißt über die Bewerbung auf eine freie Stelle, da zurzeit in allen Bundesländern ein Mangel an Lehrkräften herrscht. Aus den Befragungen von Gewerkschaftsvertretern war jedoch ersichtlich, dass in der Vergangenheit das Ländertauschverfahren eine dominantere Rolle spielte. Es ist daher noch nicht abzusehen, wie sich das Wechselverfahren bei einer Verschlechterung der Stellensituation in den Bundesländern bewährt, speziell im Hinblick auf den zurzeit sehr flexiblen Umgang mit den unterschiedlichen Ausbildungen in den Bundesländern. Da sich das Wechselverfahren im Lehramtsbereich jedoch im Vergleich zu dem Wechselverfahren bei der Polizei als stark standardisiert und institutionalisiert darstellt, ist eine vergleichsweise stärkere Tauglichkeit zu erwarten.

\subsection{Intransparenz und Ungewissheit als belastende Faktoren}

Eine ausgeprägte Intransparenz des gesamten Wechsels wurde über alle Beamtengruppen hinweg bemängelt. Insbesondere für den Polizeibereich mit seinem dysfunktionalen Tauschregime wurde immer wieder die Undurchschaubarkeit des Verfahrens kritisiert. Es sei nicht ersichtlich, wann ein Wechsel möglich wäre, was die genauen Voraussetzungen sind und nach welchen Kriterien ein Tauschpartner ausgewählt werden würde. Auch fühlen sich die Beamten vom Dienstherrn alleine gelassen, da dieser nicht aktiv informiere. Viele Wechsler betonten, dass ohne häufiges und hartnäckiges Nachfragen keine Informationen über den Stand des Verfahrens zu erlangen wären.

Jedoch auch bei den anderen Beamtengruppen wurde eine unzureichende Informationspolitik bemängelt. So wurde im Lehramtsbereich und bei der allgemeinen Verwaltung in mehreren Fällen nicht aktiv über die Möglichkeiten einer Ausgleichszulage zum Grundgehalt informiert. Erst auf Nachfrage und teilweise erst unter Verweis auf das Beamtenstatusgesetz wurde diese gewährt. Ähnliches gilt für weitere Leistungen, wie beispielsweise das Trennungsgeld oder die Umzugskostenerstattung. In diesen Fällen hat 
der Dienstherr nicht auf die entsprechenden Vorschriften aufmerksam gemacht, was für die Wechsler einen finanziellen Nachteil bedeutet hat.

Durch die Intransparenz des Verfahrens wird dem Beamten ein besonderes $\mathrm{Ma}$ an Flexibilität abverlangt. In vielen Fällen und auch Beamtengruppen übergreifend wurden die Befragten über ihren bevorstehenden Wechsel sehr kurzfristig informiert, sodass in vielen Fällen kaum Zeit für die Bewältigung der privaten Auswirkungen des Wechsels blieb, wie beispielsweise der Wohnungssuche, die Organisation des Umzugs und die Kindergartensuche. Dies wurde von den Betroffenen als psychische Belastung wahrgenommen.

Die verschiedenen Probleme bei einem Wechsel werden jedoch nicht in gleichem Maße als belastend wahrgenommen. Vor allem die vermeintlich harten Probleme, welche hier der Kategorie der Probleme durch Landesgegebenheiten zugeordnet wurden und einen Wechsel erschweren oder gar unmöglich machen, werden als wenig belastend wahrgenommen.

Als besonders stark belastend wurden die Probleme beschrieben, die durch das Wechselverfahren (Unsicherheit und fehlende Perspektive) bei der Polizei entstehen. In vielen Fälle wurde berichtet, dass nicht die lange Dauer der Wechselverfahren bei der Polizei das eigentliche Problem sei, sondern die damit verbundene Ungewissheit. Häufig wurde betont: Wenn der Wechseltermin feststünde, würden die Beamten auch lange Zeiten bis zum Wechsel in Kauf nehmen, dies jedoch als weniger belastend wahrnehmen. Aus diesem Grund werden die Wechselverfahren bei der allgemeinen Verwaltung und im Lehramtsbereich auch als weniger belastend empfunden. Denn hier herrscht eine stärkere Klarheit und auch Verbindlichkeit, womit die Planungssicherheit deutlich erhöht ist.

Dies erklärt auch, warum die Kategorie mit den meisten Problemen, die Kategorie der weichen Probleme aus Landesgegebenheiten, häufig als mittel belastend wahrgenommen werden. Auch hier kann der Beamte klare Regeln erkennen. Zwar sind diese für ihn in der Regel nachteilig, jedoch hat er auch hier das Gefühl der Kontrolle, sodass er selbst entscheiden kann, ob er den Wechsel zu den Konditionen vollziehen möchte oder nicht.

\subsection{Mobilitätsfördernde Faktoren bei einem Bundesländer übergreifenden Wechsel}

Im Rahmen der Fallstudien konnten auch mehrere Faktoren ausgemacht werden, die einen Wechsel erleichtern. Manche dieser Faktoren beschleunigten einen Wechsel oder machten den Wechsel auch erst möglich, wobei eini- 
ge Faktoren sogar Belastungen der wechselwilligen Beamten, die auf die bereits erwähnten Problemlagen zurückzuführen waren, abmildern konnten.

So wurde deutlich, dass ein geringes Alter der wechselwilligen Beamten einen Wechsel beschleunigen konnte. Dies hat mehrere Gründe. Zuerst ist es für einen jungen Beamten in einem noch relativ niedrigen Amt einfacher, einen Tauschpartner zu finden, da ältere Beamte in der Regel durch Familie, Eigenheim etc. stärker lokal gebunden sind und die Motivation für einen Tausch häufig nicht mehr vorhanden ist. Außerdem ist bei jüngeren Beamten der Polizei die Gefahr des Nichtbestehens der erneuten Gesundheitsprüfung geringer, an der ein Tausch scheitern könnte.

Als weiteres wichtiges Ergebnis der Fallstudien lässt sich formulieren, dass eine aktive Begleitung des eigenen Falls einen Wechsel tendenziell beschleunigen kann. So wurde mindestens in einem Fall dem wechselwilligen Beamten vom Dienstherrn bestätigt, dass der Wechsel möglich gemacht wurde, weil der Beamte sich regelmäßig und sehr hartnäckig über den Sachstand der Versetzung erkundigte. Auch in anderen Fällen wurde von den interviewten Beamten bestätigt, dass ohne ihre eigene proaktive Begleitung der angestrebte Wechsel nicht wie gewünscht vonstattengegangen wäre.

Förderlich sind auch Kontakte im neuen Bundesland, welche sich als Fürsprecher einsetzen. In einigen Fällen konnte der Wechsel durch Fürsprache im neuen Bundesland beschleunigt werden: Nach entsprechender Intervention verzichtete das aufnehmende Bundesland auf einen Tauschpartner. Ebenso gab es Fälle, bei denen Auswirkungen des Wechsels zu Lasten des Beamten rückgängig gemacht wurden. In mindestens einem Fall blieb einem Beamten eine ursprünglich vorgesehene Einstufung in ein niedrigeres Amt erspart.

Auch politische Kontakte oder die Kontaktaufnahme zur Politik zeigten sich als beschleunigendes Mittel für einen Wechsel. In mindestens zwei Fällen konnte ein Kontakt zum jeweiligen Innenminister genutzt werden, um einen Wechsel problemlos zu gestalten. Allerdings scheiterte in einem der untersuchten Fälle auch der Versuch, den Wechsel über politische Kontakte zu beschleunigen. Bei ihm stellte gerade die Nähe zur Politik ein Problem dar. Dieser Wechsel sollte auf ein Amt nach B 3 erfolgen und scheiterte aber am Koalitionspartner in der Regierung. Dies stellt jedoch einen Spezialfall dar, da auf diesen Positionen die politische Grundhaltung häufig ein Einstellungskriterium ist. Für den überwiegenden Teil der Beamten, besonders im mittleren und gehobenen Dienst, sind politische Kontakte ebenso wie die Fürsprache im aufnehmenden Bundesland tendenziell förderlich.

Schließlich haben die Fallstudien den interessanten Umstand zutage gefördert, dass eine spezialisierte und gefragte Qualifikation zwar die Aufnahmebereitschaft des Dienstherrn im neuen Bundesland substantiell erhöht. 
Gleichzeitig sorgt sie jedoch dafür, dass der abgebende Dienstherr argumentiert, dass der jeweilige Beamte gerade wegen dieser speziellen und seltenen Qualifikation unabkömmlich sei. Folglich sind die Chancen für einen Wechsel größer, wenn ein Beamter eine Standardausbildung durchlaufen hat, die ihn in vielen Bereichen einsetzbar und gleichzeitig abkömmlich macht. Beamte mit einer spezialisierten und stark gesuchten Ausbildung sollten sich folglich gut überlegen, welchen Dienstherrn sie als ersten wählen. Er wird sie so schnell nicht wieder frei geben. 


\section{Handlungsempfehlungen}

Viele Handlungsempfehlungen, welche sich aus den Erkenntnissen der Fallstudien ergeben, decken sich mit denen, die aus den Ergebnissen der Online-Umfrage abgeleitet werden können. Eine Rückkehr zum System vor der Föderalismusreform I erscheint nicht realistisch, auch wenn dies viele der ausgemachten Probleme beseitigen könnte. Insgesamt konnten im Rahmen der durchgeführten 32 Fallstudien zahlreiche Probleme aufgedeckt und im Vergleich der verschiedenen Beamtengruppen analysiert werden. Während sich nicht jede Schwierigkeit bei einem Ländergrenzen überschreitenden Wechsel lösen lässt, wurde deutlich, dass insbesondere in einem der untersuchten Bereiche vermeidbare Probleme bestehen. Bereits der Blick in einen der benachbarten Beamtenbereiche macht deutlich, wie Verbesserungen erreichbar sein könnten. Dabei sollte es vor allem um den Abbau der die wechselwilligen Polizisten treffenden Ungewissheit gehen. Denn gerade die Ungewissheit über den Ausgang eines sich mitunter jahrelang hinziehenden Wechselverfahrens wird von den Betroffenen als stark belastend empfunden. Für eine mögliche Lösung kann aus dem Tauschverfahren der Lehrer gelernt werden, welches auf einem relativ verregelten, aber auch beamtenfreundlicheren Verfahren beruht, das Planungssicherheit vermittelt. Eine entsprechende Reform des Tauschverfahrens im Bereich der Polizei ließe sich verhältnismäßig leicht umsetzen und würde für die wechselwilligen Beamten eine große Erleichterung bedeuten. Umgekehrt sollte das aus dem Polizeibereich bekannte, rigide und Unsicherheit schaffende Tauschverfahrens für Beamte der allgemeinen Verwaltung und der Finanzverwaltung unbedingt vermieden werden.

Weitergehend noch sollte erwogen werden, das reine Tauschverfahren um andere Verfahren für einen Wechsel zu ergänzen. Das Tauschverfahren kann eine wichtige Rolle spielen, es sollte jedoch nicht das alleinige oder vorwiegend genutzte Verfahren für einen Länderwechsel sein. Zu groß sind die aufgedeckten Probleme, zu dysfunktional die Wirkungen von Maßnahmen gegen pathologische Entwicklungen, wie sie sich durch die Zahlungen für Tauschpartner zeigen. Hingegen scheint eine grundsätzlich offene Personalpolitik beamtenfreundlicher als rigide Tauschverfahren zu sein. Die Freigabeerklärung für einen wechselwilligen Beamten könnte auch ohne Tauschpartner erfolgen. Eine frei werdende Stelle sollte neu ausgeschrieben werden. Dies kann natürlich dazu führen, dass ein Beamter mit exakt den gewünschten Qualifikationen nicht gefunden werden kann, dennoch ist eine personalpolitische Bereicherung durch andere gegebene Qualifikatio- 
nen durchaus denkbar. In entsprechende Überlegungen sollte einbezogen werden, dass die Beamten vorwiegend aus privaten Gründen das Bundesland wechseln wollen. Mit anderen Worten, hinter dem beruflichen Wechselwunsch stehen persönliche Lebensumstände und Schicksale. Und dauerhaft unzufriedene Beamte können nicht im Interesse der Dienstherren sein.

Sollte eine grundlegendere Veränderung des Tauschverfahrens im Bereich der Polizei verworfen werden, sollte wenigstens versucht werden, die festgestellten negativen Nebenwirkungen einzufangen. Wie dargestellt, leiden Beamte, die einen Antrag auf Versetzung gestellt haben, unter Benachteiligungen bei Beförderungen und Weiterbildungen. Während nachvollziehbar ist, dass angesichts begrenzt vorhandener Beförderungsstellen vor allem diejenigen befördert werden, die dem Dienstherrn noch länger zur Verfügung stehen, kann eine Benachteiligung bei der Teilnahme an Weiterbildungsmaßnahmen nicht überzeugend begründet werden. Allerdings verursachen Weiterbildungsmaßnahmen Kosten, die kaum ein Dienstherr auf sich nehmen möchte, wenn er nicht von den neu erworbenen Qualifikationen profitiert, sondern ggf. ein neuer Dienstherr. Um das hieraus entstehende Problem zu entschärfen, könnte sich die aufnehmende Behörde substantiell an den Kosten für alle Weiterbildungsmaßnahmen beteiligen, die ein Beamter nach Beantragung einer Versetzung, aber vor Vollzug des Wechsels in ein neues Bundesland erfährt.

Gleichfalls sollte vermieden werden, gewechselte Beamte im neuen Bundesland in eine niedrigere Besoldungsgruppe einzustufen. Notwendige Nachschulungen sollten so schnell wie möglich angeboten und realisiert werden. Von den Beamten werden solche Nachschulungen und Fortbildungen, welche als Voraussetzung an eine Einstufung in die bisherige Laufbahngruppe gekoppelt sind, überwiegend als Angebot und nicht als Hürde wahrgenommen. Alles in allem könnte eine systematische und sofortige Nachqualifizierung des jeweiligen Beamten dazu beitragen, dass ein Bundeslandwechsel nicht sogleich in einer finanziellen Einbuße für den gewechselten Beamten mündet. Gleichzeitig würde der Dienstherr von der nach der Fortbildung höheren Qualifikation des Mitarbeiters profitieren.

Auch sollte Beamten die Angst genommen werden, einen Versetzungsantrag zu stellen. Sowohl die Online-Umfrage als auch die Fallstudien haben gezeigt, dass viele Polizeibeamte vor einem Versetzungsantrag zurückschrecken oder nach der Antragsstellung negative Erfahrungen gemacht haben. Der Wunsch in ein anderes Bundesland zu ziehen, sollte nicht dazu führen, dass es zu Diskriminierungen auf der Dienststelle kommt oder der Beamte von Aufstiegen, Beförderungen und Weiterqualifizierungsmaßnahmen ausgeschlossen wird. Und sollte an dem bisherigen Tauschverfahren festgehalten werden, führen die Diskriminierungserfahrungen zu einer un- 
nötigen Verknappung an Tauschpartnern, was für das Gesamtsystem letztendlich dysfunktional ist.

Schließlich sollten die Dienstherren wechselwillige Beamte besser über ihre Möglichkeiten und Rechte informieren. Wenn beispielsweise Ausgleichszahlungen helfen können, finanzielle Härten zu vermeiden, dann sollte hierüber informiert werden. Insgesamt ist es der falsche Weg, die Hürden für einen Wechsel so hoch wie möglich zu setzen.

Hiermit geht einher, sich stärker um einen Abbau der Komplexität von Versetzungsverfahren zu bemühen. Immerhin sehen gut 58 Prozent der befragten Personalverantwortlichen im Bereich der Finanzverwaltung, 40 Prozent im Polizeibereich und gut 32 Prozent im Bereich der allgemeinen Verwaltung ziemlich komplizierte und eher komplizierte Tauschverfahren. Und selbst im Lehramtsbereich beträgt der entsprechende Wert gut 29 Prozent, wobei die maximale Ausprägung (ziemlich kompliziert) von lediglich 2,9 Prozent darauf verweist, dass Versetzungen im Lehramtsbereich vergleichsweise unproblematisch sind. Hier trägt das relativ geregelte Verfahren offensichtlich zu einer positiveren Einschätzung bei. Die Werte für alle betrachteten Verwaltungsbereiche würden sich nochmals substantiell um bis zu 30 Prozent (Polizei) bis 17,6 Prozent (Lehrer) erhöhen, wenn die Mittelkategorie „teils/teils“ hinzugenommen wird (siehe Abbildung 10). Dass Versetzungsverfahren als kompliziert wahrgenommen werden, hat natürlich mit den bereits angesprochenen unterschiedlichen materiellen Vorschriften, aber auch mit den jeweiligen Verfahren und Verfahrensdauern zu tun.

Generell ließen sich Mobilitätshemmnisse durch eine freiwillige Vereinheitlichung derjenigen Vorschriften abbauen, die hier als insbesondere den Wechsel von einem Bundesland $\mathrm{zu}$ einem anderen behindernd ermittelt wurden. Hierzu gehören nach Auffassung der befragten Personalverantwortlichen die unterschiedlichen Laufbahnsysteme in den Ländern mit einer unterschiedlichen Zahl von Laufbahngruppen. Während Länder mit einer einzigen sogenannten Leistungs- bzw. Einheitslaufbahn, die in Bayern und Rheinland-Pfalz anzutreffen sind, keine entsprechenden Hürden aufbauen, sind diese in Ländern mit drei Laufbahnen eher zu vermuten. Ob alle Länder jedoch bereit sind, den Weg der zwei genannten Bundesländer zu gehen, ist ungewiss.

Die Auswertung der durchgeführten Fallstudien verweist darauf, dass die mitunter nicht vollständige Anerkennung der Laufbahnbefähigung und die unterschiedlichen Höchstaltersgrenzen für die Verbeamtung ein Wechselhindernis bedeuten können. Auch hier sollte eine stärkere Vereinheitlichung bzw. der flexiblere Umgang mit Beamten aus anderen Bundesländern angestrebt werden. 
Die gegenseitige Anerkennung von Ausbildungen und Qualifizierungen scheint vor allem im Bereich des Lehramts und der Finanzverwaltung, aber auch der Polizei, wie die Fallstudien offengelegt haben, ein Problem zu sein. An dieser Stelle könnte folglich eine Politik ansetzen, der am Abbau von Mobilitätshindernissen gelegen ist. 


\section{Zusammenfassung und Fazit}

Im Zentrum der hiermit vorgelegten Studie stand die Frage, ob die sich auseinanderentwickelnden Laufbahngesetze in der Vollzugspraxis ein Mobilitätshemmnis für wechselwillige Beamte bedeuten (siehe Kapitel 1.1). Diese und weitere Unterfragen wurden in einem dreistufigen Verfahren versucht zu beantworten. Da Ausgangspunkt der Fragestellung die mit der Föderalismusreform I des Jahres 2006 veränderten Gesetzgebungskompetenzen im Bereich der Beamten sind und begründete Ausgangsüberlegung war, dass sich die Laufbahngesetze im Vergleich der Bundesländer zunehmend fragmentiert darstellen, lag als erster Schritt eine Vorschriftenanalyse nahe. Ihr Ergebnis wurde ausführlich an anderer Stelle dokumentiert (Burmester 2015). In diesen Band ging eine Kurzfassung ein (siehe Kapitel 4). Mit ihr konnte erstens gezeigt werden, dass auf der Normebene tatsächlich eine zunehmende Fragmentierung zu konstatieren ist. Zweitens gelang es, Thesen und Hypothesen zu möglichen Entwicklungen und Zusammenhängen zu formulieren (siehe Kapitel 4.5). Ihre systematische Überprüfung erfolgte im Rahmen einer online-gestützten Befragung von personalverantwortlichen Beamten in den Bereichen allgemeine Verwaltung, Finanzverwaltung, Polizei und Lehramt. Diesem Ziel diente auch die Durchführung und systematische Auswertung von insgesamt 32 Fallstudien, die konkrete Bundesländergrenzen übergreifende Wechsel von Beamten zum Gegenstand haben. Wegen der grundsätzlichen Offenheit dieser sowohl quantitativ als auch qualitativ ansetzenden Methode konnten auch über die ursprünglichen Thesen und Hypothesen hinausgehende Erklärungszusammenhänge aufgedeckt werden.

Im Folgenden sollen zunächst die aus der Vorschriftenanalyse generierten Thesen und Hypothesen knapp wiederholt werden, um anschließend bei der Darlegung der aus der Online-Umfrage und den Fallstudien gewonnenen Ergebnisse auf einer klaren Strukturierung aufsetzen zu können. Mit den Thesen und Hypothesen wurden gleichzeitig die dem Projekt zugrundeliegenden Unterfragen vollständig berücksichtigt. Die Vorschriftenanalyse abschließend konnten die folgenden Thesen formuliert werden:

- These Nr. 1: Unterschiedliche Besoldungshöhen in den Ländern bilden einen merklichen Anreiz für einen Wechsel des Bundeslandes.

- These Nr. 2: Ein attraktives Dienstrecht mit attraktiven Weiterbildungsund Aufstiegsmöglichkeiten stellt einen merklichen Anreiz für einen Wechsel des Bundeslandes dar. 
- These Nr. 3: Die Unterschiedlichkeit der Vorschriften in den Bundesländern verkomplizieren einen Bundesländer übergreifenden Wechsel von Beamten.

- These Nr. 4: Die im Vergleich der Bundesländer unterschiedliche Zahl an Laufbahngruppen bedeutet keine nennenswerten Hindernisse bei einem Wechsel von einem Bundesland in ein anderes.

- These Nr. 5: Die Nichtanerkennung von erworbener Ausbildung kann zu einem Mobilitätshemmnis werden.

- These Nr. 6: Die zur Anwendung gelangenden Tauschverfahren machen einen Wechsel des Bundeslandes aufwendig.

Zur Engführung des Vergleichs zwischen den seit längerem föderalisierten Bereichen Polizei und Lehramt einerseits sowie den erst seit 2006 föderalisierten Bereichen Finanz- und allgemeine Verwaltung andererseits wurden darüber hinaus drei Hypothesen aufgestellt:

- Hypothese A: Von den Beamten in den schon seit längerem föderalisierten Bereichen Polizei und Lehramt wird ein Versetzungsverfahren als komplizierter eingeschätzt als von den Beamten der erst kürzlich föderalisierten Bereiche allgemeine Verwaltung und Finanzverwaltung.

- Hypothese B: Von den Beamten in den schon seit längerem föderalisierten Bereichen Polizei und Lehramt wird ein Tauschverfahren als aufwendiger eingeschätzt als von den Beamten der erst kürzlich föderalisierten Bereiche allgemeine Verwaltung und Finanzverwaltung.

- Hypothese C: Bei den bislang wenig föderalisierten Bereichen der allgemeinen Verwaltung und der Finanzverwaltung ist mit einer stärkeren Zunahme der Probleme bei einem Bundesländergrenzen übergreifenden Dienstherrenwechsel zu rechnen als bei den bereits länger föderalisierten Bereichen Polizei und Lehramt.

Die Antwort auf die erste zentrale Forschungsfrage, die auf die stärksten Motive für einen Wechsel des Bundeslandes gerichtet ist und in den Thesen Nr. 1 und Nr. 2 verdichtet wurde, verweist auf die hohe Bedeutung privater Gründe für einen Bundeslandwechsel. Für die allgemeine Verwaltung werden diese von ca. 70 Prozent und für die übrigen Verwaltungsteile von ca. 87 Prozent der Befragten als Hauptgrund gesehen. Deutlich zurück bleiben hingegen berufliche Gründe (ca. 12 Prozent für die allgemeine Verwaltung) und wirtschaftliche Gründe wie die bessere Besoldung (knapp 6 Prozent bei der allgemeinen Verwaltung). Damit können die Thesen Nr. 1 und Nr. 2 zwar nicht verworfen werden. Denn der von einer höheren Besoldung und besseren Aufstiegsmöglichkeiten ausgehende Anreiz zu einem Wechsel ist tatsächlich ,merklich; aber im Vergleich zu den privaten Gründen doch rela- 
tiv schwach ausgeprägt. Damit ergeben sich entsprechende Möglichkeiten, sich von anderen Bundesländern zu unterscheiden und damit für die Beschäftigten ggf. attraktiver zu sein als andere Bundesländer. Wichtige Anreize für einen Wechsel des Bundeslandes unterliegen also insgesamt der Gestaltung durch die Bundesländer. Im Vergleich mit privaten Gründen, das Bundesland zu wechseln, spielen die von den Dienstherren beeinflussbaren Faktoren jedoch immer noch eine untergeordnete Rolle. Die Anreize für einen Bundesländer übergreifenden Wechsel des Dienstherrn sind daher noch weitgehend unabhängig vom Laufbahnrecht und der Föderalismusreform zu sehen.

In direkter Beantwortung der zweiten zentralen Forschungsfrage kann auf Basis der Ergebnisse der Online-Umfrage festgestellt werden, dass die sich auseinanderentwickelnden Vorschriften in Teilen als Mobilitätshemmnisse gesehen werden und geeignet seien, Wechsel von einem Bundesland in ein anderes zu behindern. Insbesondere die unterschiedlichen Laufbahnsysteme werden von den befragten Personalverantwortlichen zumindest als problematisch für Bundesländer übergreifende Dienstherrenwechsel wahrgenommen. Diese Entwicklung ist unmittelbar auf die Föderalismusreform I des Jahres 2006 zurückzuführen.

Sich aus den einschlägigen Vorschriften ergebende Mobilitätshemmnisse setzen vorwiegend an der individuellen Ebene des wechselwilligen Beamten an. Die Persönlichkeit oder auch der gesundheitliche Zustand des Tauschpartners, aber auch die Unabkömmlichkeit des aus Sicht des Dienstherrn eigenen Beamten sind dabei wichtige Faktoren, die zum Scheitern von Versetzungsverfahren führen können.

Werden die unterschiedlichen Vorschriften jenseits des konkreten Laufbahnsystems in den Blick genommen, wird These Nr. 3 vorläufig bestätigt. Angesichts von ca. 21 Prozent der Befragten, die in den unterschiedlichen Vorschriften der Bundesländer eher keinen Grund für eine Verkomplizierung von Wechseln sahen, fällt die Bestätigung der These jedoch nicht sehr stark aus.

Etwa die Hälfte der Befragten schätzten die unterschiedlichen Laufbahnsysteme als problematisch für einen Bundesländer übergreifenden Dienstherrenwechsel ein. Auch in den offenen Antworten wurde mehrfach auf die Unvereinbarkeit von Laufbahnsystemen, aber auch von Laufbahngruppen hingewiesen. Die Vorschriftenanalyse hatte jedoch ergeben, dass sich die Systeme in ihrer vermeintlichen Unterschiedlichkeit noch immer sehr ähnlich sind und nicht zu gravierenden Hürden führen sollten. Ganz offensichtlich klafft unsere eigene Einschätzung und die der personalverantwortlichen Experten aus der Verwaltung auseinander. Folglich kann These Nr. 4 nicht bestätigt werden. 
Weitgehend positiv ist die derzeitige Einschätzung über das Maß an Anerkennung von Ausbildung und Qualifizierungen der wechselwilligen Beamten, womit These Nr. 5 nicht bestätigt werden konnte. Allerdings wurde für das Lehramt und die Finanzverwaltung die Kategorie „teils/teils“ in substantiellem Umfang gewählt, was auf gewisse Schwierigkeiten hindeutet und somit die These nicht vollständig abzulehnen ist (siehe Abbildung 12).

Schließlich gilt es festzuhalten, dass das Tauschpartnersystem aufgrund seiner weitgehend starren Struktur wenig Spielraum für Landesgrenzen überschreitende Mobilität lässt. Manche Wechselwünsche münden nicht einmal in einen entsprechenden Antrag ein. Gründe hierfür sind erstens die erwarteten niedrigen Erfolgschancen und zweitens die Befürchtung, nach Antragstellung diskriminiert zu werden. Letzteres wirkt sich vor allem negativ aus, wenn die Wartezeit bis zur tatsächlichen Versetzung mehrere Jahre beträgt oder diese gar scheitert. Dieser Umstand entfaltet vor allem im Bereich der Polizei Wirksamkeit. Damit konnte die These Nr. 6 insgesamt bestätigt werden.

Hypothese A konnte durch die Ergebnisse der Online-Umfrage nicht bestätigt werden. Das Versetzungsverfahren wurde von den personalverantwortlichen Beamten der seit längerem föderalisierten Bereiche Polizei und Lehramt nicht als komplizierter eingeschätzt als von den personalverantwortlichen Beamten der erst seit kurzem föderalisierten Bereiche Finanzund allgemeine Verwaltung. Gerade im Lehramtsbereich wird das Wechselverfahren als eher unkompliziert und im Bereich der Finanzverwaltung als vergleichsweise kompliziert wahrgenommen. Im Mittelbereich liegen die allgemeine Verwaltung und die Polizei, wobei die allgemeine Verwaltung näher an der Finanzverwaltung liegt und die Polizei näher am Lehramtsbereich. Möglicherweise spielt hier der Gewöhnungseffekt eine gewisse Rolle. Dass das Verfahren für den Lehramtsbereich als weniger kompliziert eingeschätzt wird als für die Polizei, könnte an dem klareren und beamtenfreundlicheren Verfahren im Lehramtsbereich liegen (siehe hierzu die Ergebnisse aus dem Fallstudienkapitel, insbesondere Kapitel 6.3).

Entsprechend kann auch die Hypothese B nicht bestätigt werden, der zufolge die bereits seit längerem föderalisierten Bereiche den Aufwand des Tauschverfahrens als höher einschätzen. Hier sind es wiederum die weniger erfahrenen Bereiche, die einen hohen Aufwand bei der Suche eines Tauschpartners sehen (siehe Abbildung 11).

Hypothese C, mit der formuliert wurde, dass die Fragmentierung der Vorschriften vor allem in der Finanz- und der allgemeinen Verwaltung zu einer Zunahme der Probleme bei einem Bundesländergrenzen übergreifenden Wechsel führt, da der Polizei- und der Lehramtsbereich bereits vorher föderalen Strukturen unterlagen, konnte hingegen bestätigt werden. Im 
Zeitverlauf sehen etwa jeweils 20 Prozent der Befragten aus der allgemeinen Verwaltung und der Finanzverwaltung eine Zunahme der Probleme bei einem Bundesländergrenzen überschreitenden Wechsel. Die Zahlen für die Polizei liegen mit knapp 14 Prozent deutlich darunter und für den Lehramtsbereich mit 4 Prozent sehr deutlich (siehe Kapitel 5.4). Dieses Ergebnis, mit dem auf Veränderungen im Zeitverlauf abgehoben wird, ist von besonderem Interesse. Lässt sich doch aus ihm ablesen, dass gerade in den neu föderalisierten Bereichen die Probleme zugenommen haben. Es ist zu vermuten, dass dies auf die sich auseinanderentwickelnden Vorschriften zurückzuführen ist.

Die nachfolgend zusammengefassten Erkenntnisse aus der Auswertung der Fallstudien weisen keinen repräsentativen Charakter auf. Da sich einige der Ergebnisse mit denen der Online-Umfrage decken, kann jedoch von einer gewissen Generalisierbarkeit ausgegangen werden. Die gegenseitige Validierung der Ergebnisse war wie erinnerlich ein Grund für die gewählte methodische Triangulation (siehe Kapitel 2). In Übereinstimmung mit den Ergebnissen aus der Online-Umfrage lässt sich auch für die Fallstudien festhalten, dass der Hauptmotivationsgrund für einen Wechsel meist persönlicher Natur ist. Nur eine Minderheit der Beamten wechselt das Bundesland, weil sie annehmen, dort bessere Bedingungen durch ein attraktiveres Dienstrecht vorzufinden.

Unabhängig von der Motivation für einen Wechsel ist ein solcher durchaus problembehaftet. Die entsprechenden Schwierigkeiten wurden in zwei Kategorien eingeteilt. Erstens resultieren Probleme aus den spezifischen Landesgegebenheiten und zweitens entstehen Probleme durch das Wechselverfahren, die durchaus massiv auftreten können. Die erste Kategorie lässt sich wiederum in harte und vergleichsweise weiche Probleme differenzieren, wobei argumentiert wird, dass sich keine eindeutige Trennungslinie zwischen diesen beiden Subkategorien ziehen lässt. Ob ein Problem als eher hart oder als eher weich wahrgenommen wird, hängt mit den je spezifischen Handlungsorientierungen und objektiven Lebensumständen der Beamten zusammen. Will ein Beamter beispielsweise dringend aus familiären Gründen wechseln, wird er bereit sein, Einbußen bei der Besoldungshöhe in Kauf zu nehmen. Anders wird dies bei einem Beamten sein, der schlicht in die Heimat zurück möchte, aber substanzielle finanzielle Verpflichtungen hat. Probleme, die als hart wahrgenommen werden, stellen gewichtige Hürden bei einem Wechsel dar. Solche Schwierigkeiten ergeben sich beispielsweise aus einem unterschiedlichen Berechnungsverfahren bei der erreichten Erfahrungsstufe, aus unterschiedlichen Versorgungssystemen im Krankheitsfall oder der eingeschränkten Anerkennung der erworbenen Laufbahnbefähigung. 
Gravierender sind die Schwierigkeiten, die sich aus den Spezifika der unterschiedlichen Wechselverfahren ergeben. Die größten Probleme scheinen bei der Polizei mit dem dort zur Anwendung gelangenden Tauschverfahren zu bestehen. Wie die Fallstudien halfen aufzudecken, dauert es mitunter Jahre, bis ein Tausch zustande kommt, ohne dass der antragstellende Beamte in irgendeiner Weise Planungssicherheit erlangt. Während dieser Wartezeit ist er in aller Regel von Beförderungen und Fortbildungen abgeschnitten und erhält relativ schlechte Beurteilungen, die dann auch für verspätete Beförderungen am neuen Dienstort ursächlich sind. Diesen Nachteilen versuchen Beamte dadurch zu entgehen, dass sie einen Antrag auf Versetzung erst stellen, wenn sie einen Tauschpartner gefunden haben. Das hat nun wiederum den dysfunktionalen Effekt, dass nicht alle wechselwilligen Beamten im System gemeldet sind und damit die Zahl der offiziell zur Verfügung stehenden Tauschpartner künstlich verknappt wird.

Die Lage auf dem Tauschmarkt ist so prekär, dass von den wechselwilligen Beamten mitunter substantielle Geldbeträge, kaschiert als „Umzugskostenpauschale“, für Tauschpartner gezahlt werden. Die Länder versuchen dem durch eine willkürliche Verwendung der benannten Tauschpartner entgegen zu wirken, was wiederum Frustrationen bei denjenigen fördert, welche die Tauschpartner beigebracht haben. Weitere Probleme kommen durch die informellen Absprachen der Bundesländer über die Organisation des Wechselverfahrens hinzu. Im Vergleich zum bei der allgemeinen Verwaltung und im Lehramtsbereich vorherrschenden Wechselverfahren sind bei der Polizei die meisten Probleme zu beobachten. Sie werden von den Betroffenen als besonders belastend beschrieben, insbesondere, weil diese nicht planbar oder hervorsehbar sind und somit große Unsicherheiten hervorrufen.

Für einen Wechsel sind ein geringes Alter, eine proaktive Begleitung des eigenen Verfahrens sowie eine durchschnittliche, wenig spezialisierte Ausbildung hilfreich. Ebenso können politische Kontakte sowie Fürsprecher im aufnehmenden Bundesland dienlich sein.

Bevor hier die Ergebnisse in einen weiteren Kontext gestellt werden, gilt es nochmals zu betonen, dass es vorwiegend private Gründe sind, welche Beamte einen Wechsel des Dienstherrn anstreben lassen. Dabei gilt dies insbesondere für Lehrer, Finanzbeamte und Polizisten. Einzig für Beamte der allgemeinen Verwaltung spielen auch andere Aspekte wie eine bessere Besoldung und Versorgung oder bessere Beförderungschancen eine gewisse Rolle. So ist jedenfalls die Einschätzung der befragten Personalverantwortlichen. Dies bedeutet dann auch, dass die Dienstherrn keine unnötigen Hürden aufrichten müssen, um die eigenen Beamten zu halten. Vielmehr verursacht jede Hürde, die durch inkompatible Vorschriften oder restriktive Tauschverfahren entstehen, eine unnötige persönliche Belastung der wechselwilligen 
Beamten. Gleichwohl sollten alle Dienstherrn mit Blick auf die am Ende ihrer Ausbildung stehenden und die erst in die Ausbildung eintretenden zukünftigen Beamten ein attraktives Umfeld schaffen. Denn bei diesen Gruppen greifen die inner- und außerbehördlichen Hürden nur zu einem geringen Maße oder überhaupt nicht. Sich attraktiv aufzustellen, ist für den öffentlichen Dienst als Ganzes - also für die Bereiche der Beamten und der Tarifbeschäftigten - angesichts der weiter oben skizzierten demographischen Entwicklung und der bereits jetzt ca. 300.000 unbesetzten Stellen existentiell wichtig. Dabei konkurrieren die Länder nicht nur untereinander und mit dem Bund, sondern vor allem mit der Privatwirtschaft, die gleichfalls unter einem Mangel an qualifizierten Arbeitskräften leidet. Zu bedenken ist auch, dass im Bereich des öffentlichen Dienstes in Grenzregionen und in der alten und neuen Hauptstadt nicht einmal ein Umzug notwendig ist. Es bestehen bspw. Optionen in ein benachbartes Bundesland zu wechseln bspw. von Berlin nach Brandenburg - oder aber vom Landesdienst in den Bundesdienst (siehe Kummert, 2018, S. C3). Gerade der Bund besoldet klar besser als die Länder Berlin und Nordrhein-Westphalen und er scheint auch durchaus Landesbeamte abzuwerben (siehe Kathke 2018, S. 108). Um halbwegs attraktiv zu bleiben, konnte es sich das Land Berlin auch nicht auf Dauer erlauben, aus dem Tarifvertrag der Länder auszusteigen und das Besoldungsschlusslicht zu bilden. Zahlte das Land Berlin im Jahre 2010 ca. 94 Prozent des Tarifvertrags der Länder, sorgten vergleichsweise hohe Abschlüsse für Berlin dafür, dass die Entgeltordnung dieses Landes ab dem 1. Dez. 2017 zu 100 Prozent dem Tarifvertrag der Länder entsprach. Allerdings wurde auch die wöchentliche Arbeitszeit mit 39:24 Stunden an den Durchschnitt der Bundesländer angepasst. Hierfür war eine Erhöhung der wöchentlichen Arbeitszeit um 24 Minuten notwendig. ${ }^{25}$ Entsprechend hatte Berlin im Jahre 2014 in allen betrachteten Besoldungsgruppen (A 5, A 9 und A 13) noch das Schlusslicht inne (DGB 2014, S. 11-15). Wie in Kapitel 3 deutlich wird, hat das Land Berlin dieses mittlerweile weitergereicht, um ein halbwegs attraktiver Arbeitgeber zu bleiben. Ähnliche Überlegungen gelten für die Praxis der Verbeamtung. Auch hier lernt das Land Berlin gerade zaghaft hinzu. Noch immer gilt höchste Zurückhaltung bspw. bei der Verbeamtung von Lehrkräften mit der Folge, dass diese in Bundesländer wechseln, in denen Lehrer noch verbeamtet werden (Gellner 2019). Wollen verbeamtete Lehrkräfte von anderen Bundesländern nach Berlin wechseln, müssen sie mindestens fünf Jahre lang verbeamtet sein, wenn sie ihren Be-

25 Siehe Öffentlicher Dienst.Info, Tarifvertrag für den Öffentlichen Dienst der Länder (TV-L), TV Land Berlin, https://oeffentlicher-dienst.info/tv-1/berlin/, zuletzt abgerufen am 04.02.2020. 
amtenstatus beibehalten wollen ${ }^{26}$. Rein rechtlich dürfen Lehrkräfte nach einem Urteil des Bundesverfassungsgerichts verbeamtet werden, obwohl sie nach Einschätzung des Gerichts nicht schwerpunktmäßig hoheitliche Aufgaben wahrnehmen ${ }^{27}$. Der Staat kann jedoch wegen der hohen Bedeutung des nach Art. 7 GG gegebenen staatlichen Erziehungsauftrages entscheiden, Lehrkräfte zu verbeamten. Zu dieser Frage hat der Berliner Senat jedoch mit dem Ziel von Personalkosteneinsparungen seit 1995 eine restriktive Linie entwickelt, die nicht nur im Lehramtsbereich wirksam wurde und die auch fortgesetzt wird (Bochmann 2019a, S. 108 f.). Seit 2003 werden neu eingestellte Lehrkräfte nicht mehr verbeamtet. Mittlerweile wird allerdings auch in Berlin darüber diskutiert, die Möglichkeiten in vollem Umfang zu nutzen, die $\$ 3$ Abs. 2 Nr. 2 BeamtStG in Verbindung mit Art. 33 Abs. 4 GG einräumt: Aufgaben, die aus Gründen der Sicherung des Staates und des öffentlichen Lebens nicht ausschließlich privatrechtlich Beschäftigten übertragen werden dürfen, können die Berufung in ein Beamtenverhältnis begründen (siehe auch Bochmann 2019b, S. 149). Umstritten ist es jedoch, die Verbeamtung als Personalgewinnungs- und -bindungsmaßnahme zur Attrahierung von Mitarbeitern in Mangelberufen einzusetzen. Rechtlich zulässig sei dies, jedoch mutmaßlich als isolierte Maßnahme kaum zielführend (Bochmann 2019b, S. 155). Man darf gespannt sein, ob der Berliner Senat in der Lage ist, seine restriktive Verbeamtungspraxis angesichts des ausgeprägten Mangels an qualifiziertem Personal durchzuhalten. Wenn etwa die Hälfte der Lehrer einer Grundschule von Berlin-Schöneberg nach Brandenburg wechseln will, weil dort verbeamtet wird (Gellner 2019), scheint vom Beamtenstatus doch eine gewisse Attraktivität auszugehen (siehe auch Kummert 2018, S. C3). Denn trotz der erwähnten Arbeitszeiterhöhung bei Berliner Tarifbeschäftigten kann formuliert werden, dass die Situation auf dem Arbeitsmarkt den Sparwillen der Länder durchaus wirksam einschränkt.

Etwas weiter gefasst zeigt sich also, dass die Wirkungen des Wettbewerbsföderalismus begrenzt sind. Dies hat sich auch bereits bei der Analyse der Besoldungshöhen gezeigt (Kapitel 3). Bei der Besoldungsgruppe A 7 sticht einzig Bayern mit einer vergleichsweise sehr guten Besoldung hervor. Natürlich muss auch der Bund als sehr gut besoldende Körperschaft in diesem Zusammenhang erwähnt werden, was auch im Hinblick auf die Besoldungsgruppen A 9 und A 13 gilt. Allerdings erfolgte in Kapitel 3 eine Konzentration auf die Länder. Bei Besoldungsgruppe A 9 besoldet dann nicht nur Bayern vergleichsweise gut, sondern auch Sachsen. Noch deutlicher wird die Sonderstellung dieser beiden Länder bei der Besoldung nach A 13.

26 Senatsverwaltung für Bildung, Jugend und Familie, Berlin, I B 1.2 - 20.01.2020.

27 BVerfG, Urteil des Zweiten Senats vom 12. Juni 2018-2 BvR 1738/12 -, Rn. (1-191), 188. 
Umgekehrt bedeutet dies natürlich auch, dass alle anderen Länder vergleichsweise schlecht besolden, aber auch, dass sie bei der Besoldung nicht sehr weit auseinanderliegen. Allerdings klafft zwischen dem am drittbesten besoldenden Land Thüringen und dem am schlechtesten besoldenden Land Hessen in A 9 immerhin ein Betrag von 1.621 Euro.

Die unterschiedliche Attraktivität einer Beschäftigung in den verschiedenen Bundesländern kann, obwohl die meisten aktiv angestrebten Wechsel von Beamten auf private Gründe zurückzuführen sind, mittelfristig zu einer Verschärfung regionaler Disparitäten führen. Wenn gerade junge, mobile Lehrer, Finanzbeamte, Polizisten und Mitarbeiter der allgemeinen Verwaltung dorthin gehen, wo ihnen die besten Bedingungen geboten werden, werden angesichts der demographischen Entwicklung und den mit ihr einhergehenden Mangel an qualifizierten Arbeitskräften die weniger attraktiven Regionen nicht die vielleicht etwas weniger qualifizierten Beamten abbekommen, sondern gar keine mehr (siehe Dose et al. 2018, S. 7). Die nicht zu besetzenden offenen Stellen werden dann in den als unattraktiv empfundenen Regionen ihre besondere Wirkkraft entfalten. Relativ leicht wird dies an massivem Unterrichtsausfall, dem flächendeckenden Einsatz von pädagogisch wenig qualifizierten Quereinsteigern als Lehrer ${ }^{28}$ sowie zurückgehender Sicherheit wegen fehlender Polizisten erkennbar sein. Etwas genauer hinsehen wird man müssen, wenn Amtsveterinäre nicht mehr den Einsatz von Antibiotika in der Tierhaltung überprüfen können, wenn Planungsund Genehmigungsprozesse steckenbleiben, wenn Steuererklärungen nicht mehr zügig bearbeitet werden können oder wenn Steuersünder keine Steuerfahndung mehr befürchten müssen und wenn man auf ein Verwaltungsgerichtsverfahren ein Jahr und mehr warten muss. Eine funktionierende öffentliche Verwaltung und Justiz sind ein Standortfaktor im internationalen Wettbewerb, um den die Bürger vieler Entwicklungsländer Deutschland beneiden. Es ist der Mühe wert, ihn zu erhalten.

28 Wenig überraschend bietet der am besten besoldende Freistaat Bayern lediglich im Bereich der beruflichen Bildung Quereinsteigern eine Chance. Offenbar ist in Bayern der Lehrermangel anders als bspw. in Nordrhein-Westfalen und Berlin weniger drückend (siehe Hallo Karriere!, https://www.hallokarriere.com/quereinstieg-lehrer/, zuletzt abgerufen am 10.02.2020). 


\section{Literatur}

Baßlsperger, Maximilian (2012): Zur Evaluation des bayerischen Leistungslaufbahngesetzes, in: Zeitschrift für Beamtenrecht, 60. Band, Heft 12, 397-403.

Becker, Andreas/Tepke, Alexia (2011): Besoldungs-Föderalismus statt einheitlichem Besoldungsrecht - eine aktuelle Bestandsaufnahme, in: Zeitschrift für Beamtenrecht 59, S. 325-333.

Behnke, Joachim/Baur, Nina/Behnke, Nathalie (2006): Empirische Methoden der Politikwissenschaft. Paderborn, München, Wien, Zürich: UTB Verlag.

Bochmann, Günter (2019a): Verbeamtung als Personalgewinnungs- und -bindungsmaßnahme für IKT-Fachkräfte (Teil 1), in: Recht im Amt, 66. Jhrg., Heft 3, 97-110.

Bochmann, Günter (2019b): Verbeamtung als Personalgewinnungs- und -bindungsmaßnahme für IKT-Fachkräfte (Teil 2), in: Recht im Amt, 66. Jhrg., Heft 4, 149-155.

Bund Deutscher Kriminalbeamter Hamburg (2008): Berlin macht unmoralisches Angebot - In Hamburg sagt man tschüss (https:/www.bdk.de/lv/hamburg/aktuelles/berlin-macht-unmo ralisches-angebot-in-hamburg-sagt-man-tsch, Download am 15.02.2017).

Burmester, Carolin (2015): Mobilität von Beamten der allgemeinen Verwaltung sowie von Lehrern und Polizisten. Vorschriftenanalyse zu den Auswirkungen der Föderalismusreform I. Duisburger politik- und verwaltungswissenschaftliche Arbeitspapiere. Nr. 2 (https://www. uni-due.de/imperia/md/content/politik/vorschriftenanalyse_burmester_2015.pdf, Download am 04.01.2020).

DGB Bundesvorstand (2006a): Bundesinnenminister Wolfgang Schäuble über Sinn und Zweck der Föderalismusreform. Wettbewerb um die besten Köpfe. Nr. 9 (http://www.beamten-m agazin.de/media/pdf/Info_09-06_final.pdf, Download am 15.02.2017).

DGB Bundesvorstand (2006b): Sonderausgabe Föderalismusreform. (http://www.beamtenstatus gesetz.de/media/pdf/sonderinfo_foederalismusreform_2006.pdf, Download am 14.02.2017).

DGB Bundesvorstand (2014): Besoldungsreport 2014. Entwicklung der Einkommen der Beamtinnen und Beamten und Bewertung der Besoldungspolitik von Bund und Ländern, DGB diskurs. Berlin.

DGB Bundesvorstand (2016): Besoldungsreport 2016. Entwicklung der Einkommen der Beamtinnen und Beamten von Bund, Ländern und Kommunen. (http://www.dgb.de/++co++cb6 f18d4-15d5-11e6-a659-52540023ef1a/DGB-Besoldungsreport-2016.pdf, Download am 07.03.2017).

DGB Bundesvorstand (2019): Besoldungsreport 2019. Die Entwicklung der Einkommen der Beamtinnen und Beamten von Bund, Ländern und Kommunen. Berlin: DGB Öffentlicher Dienst und Beamtenpolitik.

Dose, Nicolai (2013): Modernisierung der staatlichen Ordnung auf dem Rücken der Beschäftigten? Folgen der Föderalismusreform I, in: Der Personalrat 30, S. 490-493.

Dose, Nicolai (2016): Ökonomische Theorie des Föderalismus und die Föderalismusreformen. Die Bundesländer im Wettbewerb und die Beamten. In: Sommermann, Karl-Peter (Hrsg.): Öffentliche Angelegenheiten - interdisziplinär betrachtet. Forschungssymposium zu Ehren von Klaus König, Berlin: Duncker \& Humblot, S. 27-48. 
Dose, Nicolai/Reus, Iris (2016): The effect of reformed legislative competences on Länder policy-making: determinants of fragmentation and uniformity, in: Regional \& Federal Studies 26, S. 625-644.

Dose, Nicolai/Wolfes, Felix (2016): Die Höhe der Beamtenbesoldung in den Ländern. Der Versuch einer Erklärung: Parteiendifferenzhypothese oder Verschuldungsdruck? in: Zeitschrift für vergleichende Politikwissenschaft 9, S. 625-644.

Dose, Nicolai/Burmester, Carolin/Wolfes, Felix (2018): Das fragmentierte Dienstrecht der Bundesländer und die Mobilität von Beamten. Eine empirische Untersuchung zu Beamtentauschverfahren, Mobilitätsanreizen und Mobilitätshemmnissen nach der Föderalismusreform. Study Nr. 381 der Hans-Böckler-Stiftung. Düsseldorf.

GdP MV (2009): Die Polizei - Landesjournal der Gewerkschaft der Polizei Mecklenburg-Vorpommern: Ausgabe September 2009. (https://www.gdp.de/gdp/gdpmp.nsf/id/DE_GdP_M -V_Deutsche_Polizei__2009/\$file/Landesjournal_der_GdP_Mecklenburg-Vorpommern-A usgabe_September_2009.pdf, Download am 14.02.2017).

GdP Niedersachsen (2009): „Mit Volldampf vorwärts in die Vergangenheit!“. Argumentationsmappe der GdP Niedersachsen zum geplanten Dienstpostenkonzept A 11 / A 12 (https://w ww.gdp.de/gdp/gdpnds.nsf/5a419d474f3279b3c1256c09002f3b2a/dbca6b4da71ad512c125 77cc0054c68b/\$FILE/2009_11_GdP_ArgumentMappe_DP_Konzept_A11.pdf, Download am 15.02.2017).

GdP NRW (2010): Eckpfeiler für ein neues Dienstrecht in NRW. Positionspapier der Gewerkschaft der Polizei. Landesbezirk Nordrhein-Westfalen. (https://www.gdp.de/gdp/gdpnrw.n sf/id/Eckpfeiler/\$file/Dienstrecht.pdf, Download am 14.02.2017).

Gellner, Torsten (2019): Berliner Lehrer wollen kündigen - und nach Brandenburg wechseln, in: Märkische Allgemeine vom 08.04.2019, https:/www.maz-online.de/Brandenburg/Kein e-Verbeamtung-in-Berlin-Halbes-Grundschul-Kollegium-erwaegt-Wechsel-nach-Berlin, zuletzt abgerufen am 09.02.2020.

Häberle, Peter (2007): Föderalismusmodelle im kulturellen Verfassungsvergleich, in: Zeitschrift für öffentliches Recht 62, S. 39-59.

Hannack, Elke (2013): Gute Arbeit - Faire Bedingungen. Rede vor dem Schöneberger Forum in Berlin. (http://www.schoeneberger-forum.de/rueckblick/++co++0b274e3c-5847-11e3-8b 14-00188b4dc422, Download am 07.03.2017).

Hoffmann, Jan (2012): Föderalismusreform I und Zielsetzung im Hinblick auf den öffentlichen Dienst, in: Knopp, Lothar/Stürmer, Klaus/Hoffmann, Jan/Schröder, Wolfgang (Hrsg.): Besoldungs- und Versorgungsföderalismus. Beamte, Hochschullehrer und Richter in der Föderalismusfalle, Berlin: Logos Verlag Berlin, S. 19-35.

Idecke-Lux, Sabrina (2014): Quo vadis Laufbahnrecht? Schritte auf dem Weg zu einem modernen Laufbahnrecht in NRW, in: Recht im Amt 61, S. 112-117.

Jarass, Hans D./Pieroth, Bodo (2011): Grundgesetz für die Bundesrepublik Deutschland. Kommentar. 11. Auflage, München: C. H. Beck.

Kathke, Leonhard (2011): Das bayerische Leistungslaufbahngesetz, in: Recht im Amt, Heft 2/2011, 56-63. 
Kathke, Leonhard (2018): Zur Wahl des Dienstherrn im Wettbewerbsföderalismus - zugleich ein Beitrag mit praktischen Tipps zur Abwehr von Raubernennungen, in: Recht im Amt, 65. Jhrg., Heft 3, 108-113.

Keller, Berndt (2010): Arbeitspolitik im öffentlichen Dienst. Ein Überblick über Arbeitsmärkte und Arbeitsbeziehungen. Berlin: Edition Sigma.

Kropp, Sabine (2010): Kooperativer Föderalismus und Politikverflechtung. Wiesbaden: VS-Verlag für Sozialwissenschaften.

KMK (2001): Übernahme von Lehrkräften aus anderen Ländern. Beschluss der Kultusministerkonferenz vom 10.05.2001. (http://www.kmk.org/fileadmin/Dateien/veroeffentlichungen beschluesse/2001/2001_05_10-Uebernahme-von-Lehrkraeften.pdf, Download am 15.02.2017).

KMK (2002): Verfahrensabsprache zur Durchführung der Vereinbarung der Kultusministerkonferenz „Übernahme von Lehrkräften aus anderen Ländern“ vom 10.05.2001. Beschluss der Kultusministerkonferenz vom 07.11.2002 i.d.F. vom 02.03.2012. (http://www.kmk.org/file admin/Dateien/veroeffentlichungen_beschluesse/2002/2002_11_07-VerfahrensabspracheUebernahme-Lehrkraefte.pdf, Download am 15.02.2017).

KMK (2013): Regelungen und Verfahren zur Erhöhung der Mobilität und Qualität von Lehrkräften Ländergemeinsame Umsetzungsrichtlinien für die Anpassung von Regelungen und Verfahren bei der Einstellung in Vorbereitungs- und Schuldienst sowie für die Anerkennung von Studien- und Prüfungsleistungen in Studiengängen der Lehramtsausbildung. Beschluss der Kultusministerkonferenz vom 07.03.2013 i.d. F. v. 27.12.2013. (https:// www.kmk.org/fileadmin/Dateien/veroeffentlichungen_beschluesse/2013/2013_03_07-Lehr ermobilitaet.pdf, Download am 15.02.2017).

Kummert, Tim (2018): Das fliegende Lehrerzimmer, in: Frankfurter Allgemeine Zeitung vom 31.03/01.04.2018, C3.

Lorse, Jürgen (2010): Neues Dienstrecht in Bayern — Die Föderalismusreform entlässt ihre Kinder, in: Zeitschrift für Rechtspolitik 43, S. 119-121.

Maurer, Marcus/Jandura, Olaf (2014): Masse statt Klasse? Einige kritische Anmerkungen zu Repräsentativität und Validität von Online-Befragungen, in: Nikolaus, Jakob/Schoen, Harald/Zerback, Thomas (Hrsg.): Sozialforschung im Internet. Methodologie und Praxis der Online-Befragung, Wiesbaden: VS-Verlag für Sozialwissenschaften, S. 61-71.

Mayntz, Renate/Scharpf, Fritz W. (1995): Der Ansatz des akteurzentrierten Institutionalismus, in: Renate Mayntz/Fritz W. Scharpf (Hrsg.), Gesellschaftliche Selbstregelung und politische Steuerung. Frankfurt/Main und New York: Campus, S. 39-72.

Mayring, Philipp (2010): Qualitative Inhaltsanalyse. Grundlagen und Techniken. 11. Auflage, Weinheim/Basel: Beltz Verlag.

Neuerer, Dietmar (2019): Bundespolizei kann mehr als 7000 Dienstschichten nicht durchgehend besetzen, in: Handelsblatt vom 19. Oktober 2019, https:/www.handelsblatt.com/pol itik/deutschland/sicherheit-bundespolizei-kann-mehr-als-7000-dienstschichten-nicht-durch gehend-besetzen/25132048.html?ticket=ST-906122-NM92plfEYaEeRskV5KfT-ap1, zuletzt abgerufen am 11.02.2020. 
Pötschke, Manuela (2009): Potentiale von Online-Befragungen: Erfahrungen aus der Hochschulforschung, in: Nikolaus, Jakob/Schoen, Harald/Zerback, Thomas (Hrsg.): Sozialforschung im Internet. Methodologie und Praxis der Online-Befragung, Wiesbaden: VS-Verlag für Sozialwissenschaften, S. 75-89.

Reich, Andreas (2012): Beamtenstatusgesetz. Kommentar. 2. Auflage, München: C. H. Beck.

Reintjes, Matthias (2018): Dienstrechtsreformen der deutschen Länder. Eine vergleichende Politikfeldanalyse. Wiesbaden: Springer VS.

Riedel, Donata/Specht, Frank (2020): Pensionswelle trifft den Staatsdienst, in: Handelsblatt vom Montag, den 6. Januar 2020, 6-7.

Rieger, Reinhard (2014): Länderübergreifende Wechsel und Wechsel in die Bundesverwaltung, in: Metzler-Müller, Karin/Rieger, Reinhard/Seeck, Erich/Zentgraf, Renate (Hrsg.): Beamtenstatusgesetz. Kommentar, Wiesbaden: Kommunal- und Schul-Verlag, S. 153-214.

Rieger, Reinhard (2016): Betr.: Die Föderalismusreformen und die Beamten", Projekt-Nr. 2014738-4 - "Dienstherrenübergreifende Versetzungen". Pers. Email. 02.05.2016.

Schneider, Karsten (2016): Folgen für die Beschäftigten, in: Der Personalrat 33, S. 16-19.

Schnellenbach, Helmut (2011): Beamtenrecht in der Praxis. 7. Auflage, München: C. H. Beck.

Schrapper, Ludger (2016): Auf zu neuen Ufern? Neues Laufbahnrecht in Nordrhein-Westfalen, in: Zeitschrift für Beamtenrecht, Jahrg. 64, Heft 12, 397-403.

Seeck, Erich/Rieger, Reinhard (2011): Neues Laufbahnrecht der norddeutschen Küstenländer, in: Recht im Amt 58, S. 1-9.

Walm, Maik/Wittek, Doris (2014): Lehrer_innenbildung in Deutschland im Jahr 2014. Eine phasenübergreifende Dokumentation der Regelungen in den Bundesländern. 2. Auflage, Frankfurt a.M.: Gewerkschaft Erziehung und Wissenschaft.

Wichmann, Manfred/Langer, Karl-Ulrich (2017): Öffentliches Dienstrecht. Das Beamten- und Arbeitsrecht für den öffentlichen Dienst, 8. Aufl., Stuttgart: Kohlhammer.

Wolff, Heinrich Amadeus (2011): Dienstrechtsreform im föderalen Staat: Einheit in Vielfalt? in: Schrapper, Ludger (Hrsg.): Dienstrechtsreform im föderalen Staat: Einheit in Vielfalt?, Baden-Baden: Nomos Verlagsgesellschaft, S. 59-80.

Yin, Robert K. (2013): Case Study Research: Design and Methods. 5. Auflage, Los Angelos/ London/New Dehli/Singapore/Washington DC: Sage Publications. 


\section{Autorinnen und Autoren}

Burmester, Carolin, M.A., war wissenschaftliche Mitarbeiterin im von der Hans-Böckler-Stiftung geförderten Projekt die „Föderalismusreformen und die Beamten. Auswirkungen der jüngsten Reformen des deutschen Föderalismus“, das am Lehrstuhl für Politikwissenschaft und Verwaltungswissenschaft der Universität Duisburg-Essen angesiedelt war.

Dose, Nicolai, Prof. Dr., ist Inhaber des Lehrstuhls für Politikwissenschaft und Verwaltungswissenschaft der Universität Duisburg-Essen. Er hat das von der Hans-Böckler-Stiftung geförderte Projekt die „Föderalismusreformen und die Beamten. Auswirkungen der jüngsten Reformen des deutschen Föderalismus" geleitet.

Wolfes, Felix, M.A., war wissenschaftlicher Mitarbeiter im von der HansBöckler-Stiftung geförderten Projekt die „Föderalismusreformen und die Beamten. Auswirkungen der jüngsten Reformen des deutschen Föderalismus“, das am Lehrstuhl für Politikwissenschaft und Verwaltungswissenschaft der Universität Duisburg-Essen angesiedelt war. Zurzeit ist er wissenschaftlicher Mitarbeiter an diesem Lehrstuhl. 
